Aspergillum. The type specimen is in the cabinet of Dr. James C. Cox of Sydney.

\title{
DESCRIPTION OF PLATE IV.
}

Fig. 1. Helix (Helicostyla) croftoni, p. 18.

2. - (Hadra) parsoni, p. 18 .

3. Bulimus (Placostylus) cuniculinsula, p. 19.

4. - (Charis) kreftii, p. 19.

5. Helix (Geotrochus) leucophcea, p. 20.

6. - - (-) brodei, p. 20.

7. - (-) choiseulensis, p. 21.

8. - (-) mendoza, p. 21.

9. Triton (Epidromus) coxi, p. 22.

10. Humphreyia coxi, p. 22.

In a second communication from Mr. Brazier, the following additional habitats of certain species of Volutida were given :--

Scapha mamilla, Gray, hitherto regarded as only from Tasmania, has been found near Lake Macquarie, New South Wales.

Scaphella marmorata, Swainson, ranges from Outer Manly Beach, near the north head of Port Jackson, northwards as far as the Clarence river.

Scapha deshayesii, Reeve, is not from the Solomon Islands, as given by Dr. Gray in his British-Museum Catalogue, but from the east coast of New Caledonia, at Uagap, and north of Balade.

Scapha punctata, Swainson, is found at intervals all along the east coast of New South Wales, from Broken Bay northwards as far as the Bellengen and Clarence rivers.

\section{January 16, 1872.}

Prof. Newton, F.R.S., V.P., in the Chair.

The Secretary read the following report on the additions to the Society's Menagerie during the month of December, 1871 :-

The total number of registered additions to the Society's Menagerie during the month of December was 64 , of which 6 were by birth, 29 by presentation, 17 by purchase, 4 received in exchange, and 8 received on deposit. The total number of departures during the same period by death and removals was 94 .

Amongst the additions three only are of sufficient interest to call for special remarks. These are :-

1. A Cuvier's Toucan (Ramphastos cuvieri), purchased Dec. 14th, being the first example of this fine species received alive by the Society. 
2. A Grey-winged Blackbird (Turdus pœcilopterus, Vigors), brought from Cashmere, and presented to the Society by Mrs. W. A. Ross, Dec. 21 st, being, as I believe, the only example of this excellent songster brought to Europe, except one previously received by the Society in 1859 *.

3. A young female Prince-Alfred's Deer, born in the Gardens Dec. 27 th, having been bred between the male presented by the Duke of Edinburgh May 5th, 1870 $\uparrow$, and the female obtained in exchange April 1st, 1871 $\downarrow$. The female was placed with the male on her arrival, and copulation took place very soon afterwards. The fawn resembles its mother, as regards marking, in every particular, and at present shows every symptom of health and strength.

The acquisition of both sexes of a Cervus previously unknown to science, and its propagation in our Gardens, must be regarded as occurrences of special interest.

I may add that, since the return of the Duke of Edinburgh to this country, I have ascertained that the male Cervus alfredi (of the history of which we were previously unacquainted) was presented to His Royal Highness by a Spanish gentleman at Manilla; so that the supposed habitat of this species (indicated P.Z.S. 1871, p. 478) is confirmed.

The following letter was read from Prof. Owen, addressed to the Secretary :-

"British Museum, 6th January, 1872.

“My DEAR Sir,-In a letter from my esteemed correspondent, Dr. Julius Haast, F.R.S. \&c., dated 'Christchurch, New Zealand, October 27th, 1871,' he informs me that 'Aptornis has been found in the Glenmark swamp; the Canterbury Museum possesses a femur identical with that figured by you.' 'Cnemiornis does not occur in the swamp' (at Glenmark) 'except very rarely, but is very frequent in the stratified, postpliocene, peaty, alluvial beds below it, exposed in the banks of a small creek.

" 'I have no doubt that, on some of the large islands at the south-western parts of this island, the Notornis is still comparatively abundant; but it is so very difficult to get there. It has always been my wish to go there for a month or so, if I could only manage to be landed by one of the steamers.'

"It is, of course, gratifying to me to receive this confirmation of the two generic types of large extinct Ralline birds in New Zealand, as it must be to you to have this acknowledgment of the aid given by the plates published in the Society's 'Transactions' in advancing our knowledge of those rare species.

"Believe me, truly yours, "Richard Owen."

“P. L. Sclater, Esq., F.R.S.,

"Sec. Zool. Soc. Lond."

* See Cat. of Vert. 1st ed. p. 50.

† See P.Z. S. 1870 , p. 381.

$\ddagger$ See P.Z.S. 1871 , p. 478. 
Mr. H. E. Dresser, F.Z.S., exhibited some skins and eggs of various species of Reguloides and Phylloscopus, and made the following remarks :-

"On the 7th of Feb. last, I had the honour to exhibit before this Society some eggs, which I then had good reason to believe were those of Reguloides superciliosus ; but since then I have ascertained that such was not the case. I have now the pleasure of bringing to your notice the true eggs of the last-named bird, collected by the well-known Indian naturalist, Mr. W. E. Brooks of Etawah. This gentleman undertook, at considerable expense, a journey to Cashmere in May last, chiefly for the purpose of finding out the breeding-haunts of this species, and was fortunate enough to procure a splendid series of eggs, obtaining likewise, in almost every instance, the old bird along with the eggs.

"I will not enter into full details of the nidification of this species (although I have received very full particulars from Mr. Brooks), as I understand from him that he has sent a paper to the Editor of 'The Ibis' on the subject, but will merely state that the Dalmatian Regulus places its nest on the ground, most generally on a bank-side, whereas its near ally, Reguloides proregulus, the eggs of which were procured by Mr. Brooks's friend, Capt. Cock, places its nest, like the Gold Crest (Regulus cristatus), high up in a fir tree. The nest of Reguloides superciliosus is dome-shaped, built of grass, and sometimes lined with hair, the entrance being at the side.

"I have also much pleasure in exhibiting an egg of Reguloides occipitalis, procured by Mr. Brooks at the same time as the abovementioned eggs of $R$. superciliosus. Mr. Brooks wrote to me some time ago, suggesting that the white eggs which I exhibited as those of $R$. superciliosus might possibly be those of $R$. occipitalis; and it is probable that he is correct in his surmise, as will be seen by comparing the egg of this species procured by Mr. Brooks with (as it now appears) the spurious eggs I exhibited on the 7 th of Feb. last. The twenty-four eggs of Reguloides superciliosus and the one egg of $R$. occipitalis were all taken by Mr. Brooks himself at Gulmurg, Cashmere, between the 31 st of May and 7 th of June last.

"The former, as will be seen from the specimens now exhibited, are white, more or less spotted with red, and in some instances purplish brown, and in character are intermediate between the eggs of our common Willow-Wren and the Chiffchaff. The egg of Reguloides occipitalis is of a pure white.

"I may also take the present opportunity of exhibiting a new European Chiffchaff, described by Mr. E. F. von Homeyer at the meeting of the German ornithologists, held in May last at Görlitz, under the name of Phyllopneuste brehmi. It is only fair to Mr. Brooks to state that he wrote to me early in March last to say that he had discovered, amongst some Warblers sent to him by the Rev. Canon Tristram, a new species which he proposed to call Phyllopneuste tristrami, which now proves to be the bird described by Mr. von Homeyer. Mr. Brooks sent to Canon Tristram a short paper describing this species at the time that he wrote to me; but, owing 
to some cause or another, it was not published, and it is now too late to do so. Directly I read Mr. von Homeyer's description of Phyllopneuste brehmi, I wrote to Mr. Brooks telling him that his bird had been, so far as I could judge, described; and he sent me his specimen, which I now exhibit, and which agrees precisely with Mr. von Homeyer's description.

"I beg also to lay before the Society another specimen of Phyllopneuste brehmi, procured near Constantinople by Mr. Robson, as also specimens of our common Chiffchaff ( $P$. rufa) from Turkey, Italy, and Great Britain, in order to show the distinctness of the present species. When sending the above specimen of $P$. brehmi, Mr. Robson wrote to me as follows :- The Chiffchaff here, I fully believe, is different from the English bird; at least I an certain the cry of the female is different. I suppose they build on the ground; but I have found no nests, though $I$ have anxiously looked for them. I have shot the bird when ready to lay, and have seen old birds feeding their young.',"

The following papers were read :-

1. A Synonymic List of the Species formerly included in the Genus Pieris; with all others described since the Subdivision of the Group by recent Authors. By A. G. Butler, F.L.S., F.Z.S., \&c.

[Received December 12, 1871.]

I have, for the last two or three years, devoted much time to the determination of the genera and species of the subfamily Pierina; and in an essay published in the third part of the 'Cistula Entomologica,' I investigated the structural characters of this group, separating it, by the neuration of the wings, form of antennæ and palpi, with other minor characters, into forty-eight genera: since the publication of this paper I have found it necessary to suppress one genus (Heliochroma), as I discovered that it was founded upon an abnormal species of Hesperocharis; I have also added two genera-Larinopoda (Trans. Ent. Soc. p. 172, Feb. 1871) and Scalidoneura (Proc. Zool. Soc. p. 250 (1871).

In the third part of my 'Lepidoptera Exotica' I commenced a revision of the species of the subfamily, with an illustrated monograph of the genus Callidryas, a paper now rather more than half finished; in March 1871 I gave a list of the species of Ixias in the 'Proceedings' of this Society, pp. 252-254; and in June a list of the species formerly included in Terias, pp. 526-541. I now propose to give a complete list of the species referred by authors to the old genus Pieris.

The genus Pieris, properly speaking, should perhaps have no place in zoological nomenclature; for if Schrank ever had a type for it, he did not fix it, and the first species that he mentions is Parnassius apollo. Latreille, I believe, subsequently fixed the type as 
$P$. cardamines, but apparently did not know what he had done, as he began his arrangement of the group in his 'Enc. Méth.' with another orange-tipped species, P. glaucippe: he certainly was not followed by subsequent authors; and it would create much confusion to follow him now. I therefore prefer to retain the first section of Boisdural's Pieris, fixing as its type the $P$. amathonte of Cramer (see 'Cist. Ent.' p. 49), and for the present rejecting the synonymous genus Perrhybris, Hübner.

In the preparation of this paper I have been much assisted by proofs of Mr. Kirby's recently published 'Catalogue of Diurnal Lepidoptera,' forwarded to me by the author before the completion of that very useful work; and it is by his express desire that I so soon supplement a portion of that publication by a revision of this group of Butterflies.

I recognize fifteen genera as having formed parts of the great genus Pieris as employed in Doubleday, Westwood, and Hewitson's 'Genera of Diurnal Lepidoptera ;' they now contain 341 described species in the following proportions:-

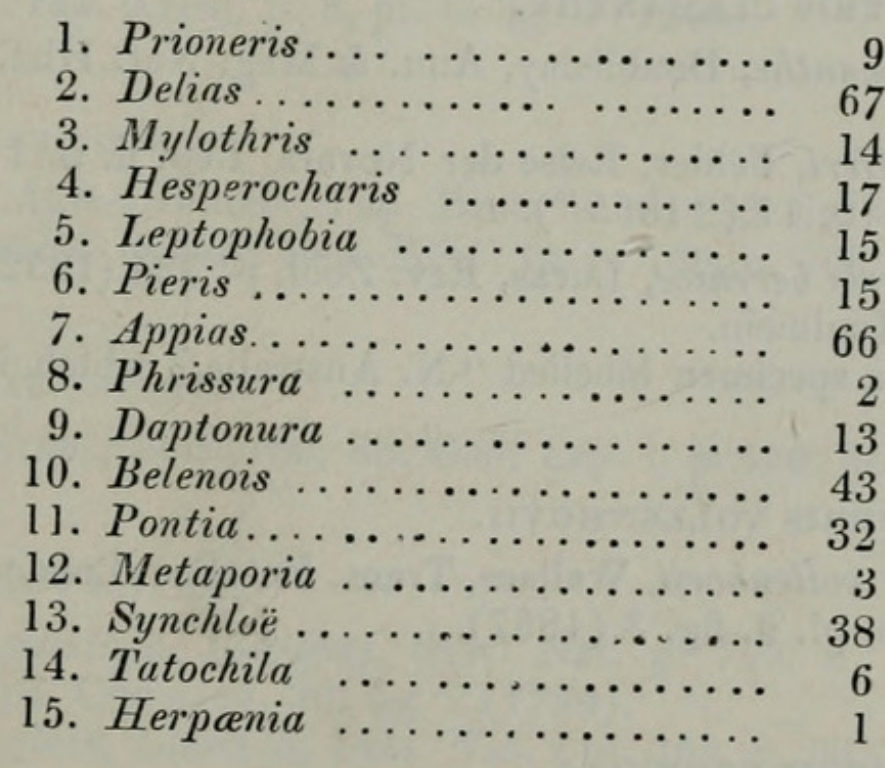

341

Genus 1. Prioneris, Wallace,

Trans. Ent. Soc. Lond. 3rd ser. vol. iv. p. 383 (1867).

\section{Prioneris thestylis.}

o. Pieris thestylis, Doubleday, in Gray's Zool. Miscell. p. 76 (1942); Gen. D. L. pl. 6. fig. 2 (1847).

N. India ; Darjeeling, E. India.

B.M.

2. Prioneris seta.

․ Pieris seta, Moore, Proc. Zool. Soc. London, p. 102, pl. 44. fig. 3 (1857).

o. Prioneris seta (part), Wallace, Trans. Ent. Soc. London, 3, vol. iv. p. 384. n. 2 (1867).

Darjeeling; Bhotan; Nepal.

I think it certain that this is not a variety of $P$. thestylis.

B.M. 
3. Prioneris watsonii.

ơ ㅇ․ Prioneris watsonii, Hewitson, Trans. Ent. Soc. London, p. 100 (1868).

o. Prioneris seta (part), Wallace, Trans. Ent. Soc. London, 3, vol. iv. p. 384 . n. 2 (1867).

Silhet.

б, B.M.

Very like a dwarfed variety of the male $P$. seta, but with a female more nearly resembling itself.

\section{Prioneris sita.}

๙. Pieris sita, Felder, Reise der Novara, Lep. ii. p. 161. n. 132, pl. 25. fig. 12 (1865).

$\checkmark$, Ceylon; $q$, Himalayas.

B.M.

The female of this species is a better mimic than the $\sigma^{*}$, and only differs from Delias eucharis in neuration and in the squareness of the marginal red spots on under surface of hind wings.

\section{Prioneris clemanthe.}

Pieris clemanthe, Doubleday, Ann. \& Mag. Nat. Hist. xvii. p. 23 (1846).

Pieris helferi, Felder, Reise der Novara, Lep. ii. p. 161. n. 131, pl. 25. figs. 10, 11 (“ 1865 ”).

Var.? Pieris berenice, Lucas, Rev. Zool. p. 324 (1852).

Silhet; Moulmein.

B.M.

We have a specimen labelled "N. Australia," which is doubtless an error.

\section{Prioneris vollenhovit.}

Prioneris vollenhovii, Wallace, Trans. Ent. Soc. London, 3, vol. iv. p. 386 . n. 6, pl. 9. fig. 3 (1867).

Borneo.

B.M.

\section{Prioneris cornelie.}

Pieris cornelia, Vollenhoven, Monogr. Pier. p. 5. no. 1, pl. 2. fig. 2 (1865).

Borneo.

B.M.

\section{Prioneris philonome.}

Pieris philonome, Boisduval, Sp. Gén. Lép. i. p. 543. n. 21 (1836).

\section{Java.}

Seems to come very near to $P$. vollenhovii; but may perhaps be a curious variety of $P$. autothisbe: I have never seen it.

\section{Prioneris autothisbe.}

๙ै. Delias autothisbe, Hübner, Samml. ex. Schmett. ii. pl. 123 (1816-24).

Java.

B.M. 
Genus 2. Delias, Hübner, Verz. bek. Schmett. p. 91 (1816).

1. Delias parthenope.

Thyca parthenope, Wallace, Trans. Ent. Soc. London, 3, vol. iv. p. 347 . n. 8 , pl. 6 . figs. $5,5 a$ (1867).

Penang.

B.M.

2. Delias ninus.

Thyca ninus, Wallace, Trans. Ent. Soc. London, 3, vol. iv. p. 347. n. 9, pl. 7. fig. 1 (1867).

Malacca.

Possibly a variation of $T$. parthenope.

3. Delias pyramus.

Thyca pyramus, Wallace, Trans. Ent. Soc. London, 3, vol. iv. p. 347 . n. 7 (1867).

Pieris thisbe, ơ, Boisduval, Sp. Gén. Lép. i. p. 449. n. 16 (1836);

Gray, Lep. Ins. Nepal, p. 8, pl. 6. fig. 1 (1846).

Nepal.

B.M.

4. Delias thysbe.

Papilio thysbe, Cramer, Pap. Exot. iii. pl. 233. fig. C (1782).

Pieris acalis, Godart, Enc. Méth. is. p. 148. n. 106 (1819).
China.

5. Delias Crithö̈.

Pieris crithö̈, Boisduval, Sp. Gén. Lép. i. p. 450. no. 18 (1836). Java.

6. Delias pasithoë.

Papilio pasithoë, Linnæus, Syst. Nat. p. 755. n. 53 (1766); Donovan, Ins. China, pl. 30. fig. 2 (179y).

Papilio aglaia, Linnæus, Syst. Nat. i. p. 465 . n. 44 (1758).

Var. Papilio dione, Drury, Ill. Ex. Ent. ii. pl. 8. figs. 3, 4 (1773). Papilio porsenna, Cramer, Pap. Exot. i. pl. 43. figs. D, E (1776); iv. pl. 352. figs. A, B (1782).

Silhet; Nepal; Moulmein; China; Borneo.

B.M.

7. Delias chrysorrhea.

Pieris chrysorrhaa, Vollenhoven, Mon. Pier. p. 6. n. 3, pl. 2. fig. 4 (1865).

Sumatra.

A species allied to D. pasithoë.

8. Delias egialea.

Papilio egialea, Cramer, Pap. Exot. ii. pl. 189. figs. D, E (1779).

Delias tyche et apriate, Hübner, Verz. bek. Schmett. p. 91 .

n. 957,958 (1816). 
Pieris pasithoë, var., Godart, Enc. Méth. ix. p. 148. n. 105 (1819).

? Papilio fusconiger et albopictus, Goeze, Beytr. i. pp. 44,45 . n. $25,26(1779)$.

Java.

B.M.

9. Delias ochreopicta.

Thyca ochreopicta, Butler, Ann. \& Mag. Nat. Hist. iv. p. 244 (1869); Lep. Exot. viii. p. 63. n. 3, pl. 24. figs. 4, 5 (1871).

Philippines (Luzon).

B.M.

10. Delias henningia.

Pontia henningia, Eschscholtz, Kotzeb. Reise, iii. p. 214, pl. 9. figs. $20 a, 20 b$ (1821).

Philippines (Manilla).

B.M.

11. Delias lucerna.

Thyca lucerna, Butler, Ann. \& Mag. Nat. Hist. 4, vol. iv. p. 243 (1869); Lep. Exot. viii. p. 62. n. 2, pl. 24. figs. 2, 3 (187l).

Philippines (Mindanao?).

B.M.

12. Delias pandemia.

Thyca pandemia, Wallace, Trans. Ent. Soc. London, 3, vol. iv. p. 346 . n. 3 , pl. 6 . figs. $4,4 a$ (1867).

Borneo.

B.M.

13. Delias ithiela.

Thyca ithiela, Butler, Ann. \& Mag. Nat. Hist. 4, vol. iv. p. 242 (1869); Lep. Exot. viii. p. 62. n. 1, pl. 24. fig. l (1871).

Darjeeling.

B.M.

14. Delias belladonna.

Papilio belladonna, Fabricius, Ent. Syst. iii. p. 180. n. 557 (1793); Donovan, Nat. Rep. i. pl. 35 (1823).

N. India?

\section{Delias horsfieldit.}

o. Pieris horsfieldii, G. R. Gray, Zool. Miscell. p. 32 (1831); Herrich.Schäffer, Ex. Scnmett. figs. 13, 14 (1850).

Nepal.

B.M.

This species seems to be distinct from $D$. belladonna; but the difference may be due to errors in Donovan's figure or to sex.

16. Delias sanaca.

Pieris sanaca, Moore, Proc. Zool. Soc.p. 103, pl. 44. fig. 4 (1857). Darjeeling.

B.M.

17. Delias aganippe.

Papilio aganippe, Donovan, Ins. New Holl. pl. 29 (1805).

King George's Sound; Adelaide; Moreton Bay.

B.M. 
18. Delias fragalactea.

Thyca frayalactea, Butler, Ann. \& Mag. Nat. Hist. 4, vol. iv. p. 243 (1869); Lep. Exot. viii. p. 64. n. 5, pl. 24. fig. 7 (1871).

N. Australia.

B.M.

This is probably a race of $D$. argenthona.

19. Delias argenthona.

Papilio argenthona, Fabricius, Ent. Syst. iii. p. 200. n. 624 (1793).

Pieris protocharis, Boisduval, Sp. Gén. Lép. i. p. 457. n. 27 (1836).

Australia; Moreton Bay; Richmond River. B.M.

20. Delias rosenbergit.

Pieris rosenbergii, Vollenhoven, Monogr. Pier. p. 11. n. 9, pl. 2. fig. 6, pl. 3. fig. 1 (1865).

Celebes.

B.M.

21. Delias lorquinit.

Pieris lorquinii, Felder, Reise der Novara, ii. p. 159. n. 128, pl. 24. figs. 9, 10 (" $1865 ")$.

Celebes.

B.M.

Allied to $D$. rosenbergii, but seems quite distinct.

22. Delias luzoniensis.

Pieris luzoniensis, Felder, Wien. ent. Monats. vi. p. 285. n. 39 (1862).

Philippines (Luzon).

B.M.

23. Delias hyparete.

Papilio hyparete, Linnæus, Mus. Lud. Ulr. p. 247 (1764); Clerck's Icones, pl. 38. fig. 2 (1764). (1782).

․ Papilio antonoë, Cramer, Pap. Exot. iv. pl. 320. figs. A, B

Java; Sumatra; Borneo; Penang; Assam.

B.M.

24. Delias hierte.

§. Delias hierte, Hübner, Zutr. Ex. Schmett. figs. 77, 78 (1818).

ㅇ, Siam ; Penang.

B.M.

25. Delias indica.

Thyca hierte, var. indica, Wallace, Trans. Ent. Soc. London, 3, vol. iv. p. 351. n. 21 (1867).

Barrackpore; Dukhun; Moulmein.

B.M.

26. Delias hemorrhea.

Pieris hamorrhaa, Vollenhoven, Monogr. Pier. p. 10. n. 8, pl. 2. fig. 5 (1865).

Banca.

B.M. 


\section{Delias stollit.}

Papilio antonoë, Stoll (nec Cramer), Suppl. Cramer, pl. 33. figs. 2, $2 b(1790)$.

China.

B.M.

Our specimens agree with Stoll's figure, which looks a distinct species from Hübner's D. hierte.

28. Delias eucharis.

Papilio eucharis, Drury, Ill. Ex. Ent. ii. pl. 10. figs. 5, 6 (1773).

Pieris epicharis, Godart, Enc. Méth. ix. p. 153. n. 122 (1819).

Papilio hyparethe, Herbst, Natursyst. Schmett. pl. 101. figs. 3-5.

N. India ; Nepal; Ceylon; Penang.

B.M.

\section{Delias mysis.}

Papilio mysis, Fabricius, Syst. Ent. p. 475. n. 138 (1775); Donovan, Ins. New Holl. pl. 21. fig. 1 (1805).

Australia; Rockingham Bay.

B.M.

\section{Delias cruentata.}

Pieris cruentata, Butler, Proc. Zool. Soc. London, p. 455, pl. 26. fig. 2 (1865).

Pieris lara, Vollenhoven (nec Boisduval), Monogr. Pier. p. 12. n. 11 (1865).

Mysol ; Port Essington.

B.M.

The insects received by Dr. Boisduval with the MS. name of $P$. lara, and supposed by him to represent a variety of $D$. mysis, can hardly be the same as $D$. cruentata, since they are described as having the black border on under surface of hind wings broader than in D. mysis; the reverse is the case with $D$. cruentata.

\section{Delias cemneus.}

Papilio caneus, Linnæus, Mus. Lud. Ulr. p. 271 (1764).

Papilio discors, Gmelin, Syst. Nat. 1, v. p. 2261. n. 881 (1788-91).

Papilio —, Zschach. Mus. Lesk. Ent. p. 88. n. 32 (1788).

Papilio antonoë, Herbst, Natursyst. Schmett. pl. 100. figs. 1-4.

Papilio anthonö̈ (sic), Herbst, Natursyst. v. Index.

Cathamia anthyparete, Hübner, Verz. bek. Schmett. p. 92. n. 961 (1816).

Var. Papilio plexaris, Donovan, Ins. New Holl. pl. 18. fig. 2 (1805).

Pieris philyra, Godart, Enc. Méth. ix. p. 150. n. 113 (1819).

N. Ceram ; and var. Amboina.

B.M.

32. Delias philotis.

Thyca philotis, Wallace, Trans. Ent. Soc. London, 3, vol. iv. p. 357 . n. 44 , pl. 8 . fig. 4 (1867).

Bouru. 
33. Delias duris.

Pieris duris, Hewitson, Exot. Butt. ii. Pier. pl. 5. fig. 34 (1861). Ceram.

34. Delias agostina.

Pieris agostina, Hewitson, Ex. Butt. i. Pier. pl. 1. figs. 1, 2 (1852).

‥ Pieris nesba, Lucas, Rev. Zool. p. 324 (1852).

N. India ; Darjeeling.

B.M.

35. Delias blanca.

Pieris blanca, Felder, Wien. ent. Monats. vi. p. 284.n. 38 (1862);

Reise der Novara, Lep. ii. p. 160. n. 130, pl. 24. figs. 6, 7 (1865).

Luzon.

36. Delias singhapura.

Thyca singhupura, Wallace, Trans. Ent. Soc. London, 3, vol. iv. p. 353. n. 29, pl. 7. fig. 2 (1867).

Bormeo; Sarawak.

B.M.

37. Delias Peribea.

Pieris peribøa, Godart, Enc. Méth. ix. p. 154. n. 124 (1819).

Java.

38. Delias themis.

Pieris themis, Hewitson, Ex. Butt. ii. Pier. pl. 5. figs. 31, 32 (1861).

Philippines.

B.M.

39. Delias gabia.

Pieris gabia, Boisduval, Voy. de l'Atrolabe, Lép. p. 49.n. 7 (1832). Waigiou.

40. Delias dice.

Pieris dice, Vollenhoven, Monogr. Pier. p. 39. n. 5, pl. 4. fig. 7 (1865).

Papua.

41. Delias ennia.

Thyca ennia, Wallace, Trans. Ent. Soc. London, 3, vol. iv. p. 355. n. 35 , pl. 7 . fig. 4 (1867).

Waigiou.

42. Delias bagoe.

Pieris bagoe, Boisduval, Voy. de l'Astrolabe, Lép. p. 49. n. 1 (1832); Wallace, Trans. Ent. Soc. 3, vol. iv. p. 355. n. 36, pl. 7. figs. $3,3 a(1867)$.

Aru, New Ireland.

Proc. Zool. Soc.-1872, No. III. 
43. Delias zeBUda.

Pieris zebuda, Hewitson, Exot. Butt. iii. Pier. pl. 7. figs. 49, 50 (1862).

Celebes.

B.M.

44. Delias Descombesi.

Pieris descombesi, Boisduval, Sp. Gén. Lép. i. p. 465 . n. 39 (1836).

Silhet; Darjeeling; Nepal ; Moulmein ; Penang.

B.M.

45. Delias STHENOBaA.

Pieris sthenobaa, Boisduval, Sp. Gén. Lép. i. p. 466. n. 37 (1836).

Moluccas.

Perhaps a variety of $\boldsymbol{D}$. descombesi; it differs only in the paler yellow and absence of red basal patch on under surface of hind wings.

46. Delias aruna.

๙. Pieris aruna, Boisduval, Voy. de l'Astrolabe, Lép. p. 48 . n. 4 (1832); ơ $q$, Hewitson, Ex. Butt. 2, Pier. pl. 3. figs. 20-22 (1861).

q. Pieris bajura, Boisduval, Voy. de l'Astrolabe, Lép. p. 48. n. 5 (1832).

Papua; Waigiou; Batchian.

\section{Delias inferna.}

Delias inferna, Butler, Lep. Exot. 8, p. 63. n. 4, pl. 24. fig 6 (1871).

North-western Australia.

B.M.

48. Delias belisama.

Papilio belisama, Cramer, Pap. Exot. 3, pl. 258. figs. A-D (1782). Java.

B.M.

49. Delias glauce.

Pieris glauce, Butler, Proc. Zool. Soc.p. 431.n. 2, pl. 25. fig. 2 (1865).

Borneo.

B.M.

50. Delias isse.

Papilio isse, Cramer, Pap. Exot. i. pl. 55. figs. E, F (1779), iv. pl. 339. figs. C, D (1782).

Papilio bicolor, Gmelin, Syst. Nat. 1, v. p. 2261 . n. 880 (178891); Zschach, Mus. Lesk. Ent. p. 88. n. 31 (1788).

Amboina, N. Ceram.

B.M.

51. Delias echo.

Thyca echo, Wallace, Trans. Ent. Soc. London, 3, vol. iv. p. 358. n. 47 , pl. 8 . fig. 3 (1867).

Bouru. 
52. Delias chrysomelena.

Pieris chrysomelana, Vollenhoven, Tijd. voor Ent. 2, vol.i. p. 57, pl. l. figs. 1, 2 (1866).

Kaioa Island.

53. Delias echidna.

Pieris echidna, Hewitson, Ex. Butt. ii. Pier. pl. 5. figs. 35, 36 (1861).

Ceram.

\section{Delias hippodamia.}

Thyca hippodamia, Wallace, Trans. Ent. Soc. London, 3, vol. iv. p. 359. n. 50, pl. 8. fig. 1 (1867).

Aru.

55. Delias dorimene.

Papilio dorimene, Cramer, Pap. Exot. iv. pl. 387. figs. C, D (1782). Papilio fuliyinosus, Gmelin, Syst. Nat. 1, v. p. 2261. n. 882

(1788-91); Zschach, Mus. Lesk. Ent. p. 88. n. 34 (1788).

Pieris ageleis, Godart, Enc. Méth. ix. p. 147. n. 103 (1819).

Ceram ; Amboina.

56. Delias dorylea.

Pieris dorylaa, Felder, Reise der Novara, Lep. ii. p. 182. n. 173 (“1865”).

Aru.

Allied to $D$. dorimene.

57. Delias pecilea.

Pieris pocilea, Vollenhoven, Monogr. Pier. p. 13. n. 13, pl. 3. fig. 3 (186.)).

Moluccas.

58. Delias candida.

Pieris candida, Vollenhoven, Monogr. Pier. p. 11.n. 10, pl. 3. fig. 2 (1865).

Batchian.

59. Delias herodias.

Pieris herodias, Vollenhoven, Monogr. Pier. p. 14. n. 14, pl. 3. fig. 4 (1865).

Gilolo.

60. Delias timorensis. (1836).

๙. Pieris timorensis, Boisduval, Sp. Gén. Lép. i. p. 459. n. 30

? ㅇ. Pieris vishnu, Moore, Cat. Lep. East I. Comp. i.p. 83. n. 168, pl. $2^{\text {a }}$. fig. 5 (1857).

Java.

B.M.

I rather doubt the specific identity of the two sexes united above, 
unless Dr. Boisduval's locality is incorrect, in which case I should suggest the adoption of Mr. Moore's name.

61. Delias nigrina.

Papilio nigrina, Fabricius, Syst. Ent. p. 475. 11. 139 (1775); Donovan, Ins. New Holl. pl. 19. fig. I (1805).

Australia; Moreton Bay; Richmond River. B.M.

62. Delias harpalyce.

Papilio harpalyce, Donovan, Ins. New Holl. pl. 18. fig. 1 (1805).

Australia ; Sydney.

B.M.

63. Delias momea.

Pieris momea, Boisduval, Sp. Gén. Lép. i. p. 477. n. 56 (1836).

Java.

B.M.

64. Delias nysa.

o. Papilio nysa, Fabricius, Syst. Ent.p. 473.n. 128 (1775).

․ Papilio endora, Donovan, Ins. New Holl. pl. 20. fig. 2 (1805). Australia ; Moreton Bay.

B.M.

65. Delias orphne.

Pieris orphne, Wallace, Trans. Ent. Soc. London, 3, vol. iv. p. 361. n. 56 , pl. 8. fig. 2 (1867).

Malacca.

66. Delias georgina.

Pieris georgina, Felder, Wien. ent. Monats. v. p. 298. n. 5 (1861); Reise der Novara, Lep. ii. p. 160. n. 129, pl. 24. figs. 4, 5 (“1865”).

Luzon.

67. Delias? furvus.

Papilio furvus, Goeze, Beytr.i. p. 182. n. 77 (1779); Seba, Thes. pl. iv. figs. 13, 14 .

Genus 3. Mylothris, Hübner.

Verz. bek. Schmett. p. 90 (1816).

1. Mylothris poppea.

Papilio poppea, Cramer, Pap. Exot. ii. pl. 110. fig. D (1779).

Papilio poppa, Herbst, Natursyst. Schmett. pl. 89. fig. 5.

Sierra Leone.

B.M.

2. Mylothris rhodope.

Papilio rhodope, Fabricius, Syst. Ent. p. 473. n. 130 (1775); Donovan, Nat. Rep. iii. pl. 86 (1825).

Ashanti ; Sierra Leone.

B.M.

3. Mylothris trimenia.

Pieris trimenia, Butler, Cist. Ent. p. 13 (1869). 
Pieris agathina, var. B, Trimen, Rhop. Afr. Austr. p. 29, pl. 2. fig. 2 (1866).

Port Natal.

B.M.

4. Mylothris agathina.

Papilio agathina, Cramer, Pap. Exot. iii. pl. 237. figs. D, E (1782).

Papilio agatina, Herbst, Natursyst. Schmett. pl. 104. figs. 4, 5. (1816).

Cathremia xantholeuca, Hübner, Verz. bek. Schmett. p. 92 . n. 964

Var. Pieris rïppellii, Koch, Indo-Austr. Lep.

Zulu ; Port Natal; Abyssinia.

B.M.

5. Mylothris phileris.

o. Pieris phileris (part.), Boisduval, Faun. de Madag. p. 17.n. 2, pl. 2. figs. 3,4 (1833).

S. Africa ; Madagascar.

Close to M. agathina.

6. Mylothris? paroreia.

Pieris paroreia, Hewitson, Trans. Ent. Soc. Lond. p. 72. n. 2 (1869).

Cape Coast.

7. Mylothris bernice.

Pieris bernice, Hewitson, Ex. Butt. iii. Pier. pl. 8. figs. 52, 53 (1866).

Gaboon.

8. Mylothris ChLORIS.

Papilio chloris, Fabricius, Syst. Ent. p. 473. n. 129 (1775); Drury, Ill. Ex. Ent. iii. pl. 32. figs. 3, 4 (1782). (1782).

Sierra Leone.

B.M.

9. Mylothris Lypera.

Pieris lypera, Kollar, Denkschr. Akad. Wiss. Wien, math.-nat. Cl. i. p. 361.n. 26, pl. 45. figs. 1, 2 (1850).

Bogota.

B.M.

10. Mylothris leptalina.

Pieris leptalina, Bates, Journ. Entom. i. p. 236. n. 7 (1861).

St. Paulo.

11. Mylothris pyrrha.

o. Papilio pyrrha, Fabricius, Syst. Ent. p. 464. n. 95 (1775).

Papilio iphigenia, Schulz. Naturforscher, ix. p. 108, pl. 2 (1776). 
§, ㅇ. Perrhybris eieidias, Hübner, Samml. ex. Schmett. pl. 12I (1816-24).

․ Papilio pamela, Cramer, Pap. Exot. iv. pl. 319. fig. A (1782). Brazil ; Tapajos.

B.M.

12. Mylothris viardi.

Pieris viardi, Boisduval, Sp. Gén. Lép. i. p. 439. n. 3 (1836).

Mexico.

13. Mylothris malenka.

Pieris malenka, Hewitson, Exot. Butt. i. Pier. pl. 1. figs. 5, 6 (18j2).

Venezuela.

B.M.

14. Mylothris LORENA.

Pieri. lorena, Hewitson, Ex. Butt. i. Pier. pl. 1. fig. 7 (1852).

Villa Nova; St. Paulo; Tunantins; Peru; Bogotá. ơ. B.M.

Genus 4. Hesperocharis, Felder,

Verhandl. Zool.-botan. Gesellsch. Wien, p. 493 (1862).

1. Hesperocharis graphites.

Hesperocharis graphites, Bates, Ent. Mo. Mag. i. p. 32. n. 12 (1864).

Guatemala.

2. Hesperocharis avivolans.

Pieris avivolans, Butler, Proc. Zool. Soc. p. 457, pl. 26. fig. 4 (1865).

Mexico.

B.M.

A local representative of $H$. graphites.

3. Hesperocharis costaricensis.

Hesperocharis costaricensis, Bates, Ent. Mo. Mag. iii. p. 49.n. 85 (1866).

Costa Rica.

4. Hesperocharis marchalit.

Pieris marchalii, Guérin, Icon. Règne Anim. Ins. texte, p. 468 (1844).

Venezuela.

B.M.

5. Hesperocharis Catogramma.

Pieris catogramma, Kollar, Denkschr. Akad. Wiss. Wien, math. Cl. i. p. 361 . n. 28 (1850).

Bolivia.

B.M.

6. Hesperocharis erota.

Pieris erota, Lucas, Rev. Zool. p. 329 (1852).

Brazil.

B.M 
7. HFSPEROCHARIS CROCEA.

Hesperocharis crocea, Bates, Ent. Mo. Mag. iii. p. 49, n. 84 (1866).

Costa Rica.

8. HeSPEROCHARIS IDIOTICA.

Helichroma idiotica, Butler, Cist. Ent. i. p. 15 (1869); Lep. Exot. ix. p. 70 , pl. 27 . fig. 2 (1871).

9. Hesperocharis lenoris.

Pieris lenoris, Reakirt, Proc. Ac. Nat. Sci. Philad. p. 336. n. 37 (1866).

Mexico.

Allied to $H$. idiotica.

10. Hesperocharis gayi.

Pieris gayi, Blanchard, Gay's Faun. Chil. vii. p. 10, pl. 1. fig. 4 (1852).

Chili.

B.M.

\section{Hesperocharis leucania.}

Pieris leucania, Boisduval, Sp. Gén. Lép. i. p. 493. no. 83 (1836). ? Pieris pasion, Reakirt, Proc. Akad. Nat. Sci. Philad. p. 336. n. 38 (1866).

Mexico.

B.M.

12. Hesperocharis anguitia.

Pieris anguitia, Godart, Enc. Méth. ix. p. 146. n. 100 (1819); Hübner, Zuträge ex. Schmett. figs. 889, 890 (1837).

Brazil.

B.M.

13. Hesperocharis Nereis.

Hesperocharis nereis, Felder, Reise der Novara, ii. p. 146. n. 112 (1865).

E. Peru; Bolivia ; Bogota.

B.M.

14. Hesperocharis nera.

Pieris nera, Hewitson, Exot. Butt. i. Pier. pl. 1. figs. 3, 4 (1852). Tapajos.

B.M.

15. Hesperocharis helvia.

Pieris helvia, Latreille, in Humboldt \& Bonpland's Obs. Zool. ii. p. 121, pl. 41. figs. 1, 2 (1811-19).

Mexico.

16. Hesperocharis hirlanda.

Papilio hirlanda, Stoll, Suppl. Cramer, pl. 35. figs. 1, $1 a(1790)$. Archidona. 


\section{Hesperocharis fulvinota.}

Hesperocharis fulvinota, Butler, Ann. \& Mag. Nat. Hist. p. 284 (Oct. 1871) ; Lep. Exot. xi. pl. 34. fig. 4 (Jan. 1872).

Back of Rio.

B.M.

Genus 5. Leрторновіа, Butler.

Cist. Ent. iii. pp. $35 \& 45$, gen. 18 (1870).

\section{Leptophobia eleone.}

Pieris eleone, Doubleday \& Hewitson, Gen. D. L. pl. 6. fig. 6(1847). Bolivia; Quito; Bogota; Venezuela.

B.M.

2. Leptophobia cesia.

Pieris casia, Lucas, Rev. Zool. p. 299 (1852); Hewitson, Ex. Butt. ii. Pier. pl. 6. figs. 43, 44 (1861).

Quito.

3. Leptophobia Semiczesia.

Pieris semicasia, Felder, Reise Nov. Lep. ii. p. 176. n. 164 ("1865").

New Granada.

Near L. pentica.

4. Leptophobia pentica.

§. Leptalis? pentica, Kollar, Denkschr. Akad. Wiss. Wien, math.-nat. Cl. i. p. 360 . n. 25, pl. 45. figs. 11, 12 (1850).

․ Pieris stamnata, Lucas, Rev. Zool. p. 330 (1852).

ơ, Venezuela; + , Bogota.

B.M.

5. Leptophobia euthemia.

Pieris euthemia, Felder, Wien. ent. Mon. v. p. 80 . n. 29 (1861). Venezuela.

Allied to $L$. pentica.

6. Leptophobia cinerea.

Pieris cinerea, Hewitson, Trans. Ent. Soc. 3rd ser. vol. v. p. 563. n. 6 (1867).

Ecuador.

7. Leptophobia philoma.

Pieris philoma, Hewitson, Equat. Lep. p. 79. n. 144 (1870).

Ecuador (Buckley).

8. Leptophobia balidia.

Pieris balidia, Boisd. Sp. Gén. Lép. i. p. 529. n. 133 (1836).

Rio Janeiro; Panamá.

9. Leptophobia elodia.

Pieris elodia, Boisduval, Sp. Gén. Lép. i. p. 529. n. 134 (1836); Hübner, Zutr. ex. Schm. figs. 853, 854 (1837).

‥ Pieris suadella, Felder, Wien. ent. Mon.v. p.79. n. 26(1861). 
Var.? Pieris eleusis, Lucas, Rev. Zool. p. 331 (1852).

Mexico; Polochic Valley; Venezuela.

B.M.

10. LEPTOPHobia heLENa.

Pieris helena, Lucas, Rev. Zool. p. 332 (1852).

Quito.

B.M.

11. Leptophobia OLYMPia.

Pieris olympia, Felder, Wien. ent. Mon. v. p. 80. n. 28 (1861). Venezuela.

12. Leptophobia tovaria.

Pieris tovaria, Felder, Wien. ent. Mon. v. p. 80. n. 27 (1861).

Venezuela.

13. Leptophobia pinara.

Pieris pinara, Felder, Reise Nov. Lep.ii. p.179.n. 169 (“1865”). Bolivia.

14. LePtophobia ARIPA.

Pieris aripa, Boisduval, Sp. Gén. Lép. i. p. 528. n. 131 (1836).

Brazil.

B.M.

15. Leptophobia pylotis.

Pieris pylotis, Godart, Enc. Méth. ix. p. 158. n. 137 (1819).

Brazil.

B.M.

Genus 6. Pieris*, Boisduval.

Sp. Gén. Lép. i. p. 434, sect. 1 (1836).

1. Pieris menacte.

Pieris menacte, Boisduval, Sp. Gén. Lép. i. p. 517.n. 116 (1836). Venezuela.

B.M.

2. Pieris? Kiçaha.

Pieris kiçaha, Reakirt, Proc. Ent. Soc. Philad. ii. p. 349. n. 9 (1863).

Honduras.

3. Pieris a maryllis.

Papilio amaryllis, Fabricius, Ent. Syst. iii. p. 189.n. 586 (1797);

Donovan, Ins. Ind. pl. 28. fig. 1 (1800).

Jamaica.

B.M.

4. Pieris josephina.

Pieris josephina, Godart, Enc. Méth. ix. p. 158. n. 136 (1819).

․ Catophaga josephine, Hübner, Samml. ex. Schm. ii. pl. 126 (1819-36).

* P. antsianaka of Ward (E. M. M. vii. p. 30, 1870) is a Nepheronia ; P. mananhari (E. M. M. vi. p. 224, 1870) a Teracolus; P. eunoma of Hopffer (Peters, Reise, pl. 23. 1, 2) belongs also to the latter genus. 
Pieris josepha, Salvin and Godman, Ann. and Mag. Nat. Hist.

s. 4 , vol. ii. p. 150 . n. 22 (1868).

o. Mexico; o 9 . Haiti.

B.M.

There is no appreciable difference between the Mexican and Haitian examples of this species.

5. Pieris bunie.

Catophaga bunia, Hübner, Samml. exot. Schmett. ii. pl. 125 (1816-36).

Pieris endeis, Godart, Enc. Méth. ix. p. 158. n. 135 (1819).

Brazil.

B.M.

6. Pieris thalö̈.

Pieris thaloë, Godart, Enc. Méth. ix. p. 156. n. 131 (1819);

Lucas, Lep. Exot. pl. 27. fig. 1 (1835).

Pieris ausia, Boisduval, Sp. Gén. Lép. i. p. 531. n. 137 (1836).

'Tapajos; Pará; Venezuela.

B.M.

7. Pieris demophile.

․ Pupilio demophile, Linnæus, Syst. Nat. 12, p. 761. n. 82 (1767).

Papilio molphea, Cramer, Pap. Exot. ii. pl. 116. fig. C (1779).

o. Papilio amathonte, Cramer, Pap. Exot. ii. pl. 116. figs. A, B (1779).

Brazil ; Tapajos; St. Paulo.

B.M.

8. Pieris calydonia.

Pieris calydonia, Boisduval, Sp. Gén. Lép. i. p. 439. n. 2 (1836). Venezuela.

B.M.

\section{Pieris marana.}

Pieris marana, Doubleday, Ann. \& Mag. Nat. Hist. xiv. p. 421 (1844) ; Hewitson, Ex. Butt. ii. Pier. pl. 6. fig. 42 (1861).

Valley of Chimborazo; W. coast of Mexico; Panamá.

B.M.

10. Pieris pandosia.

Pieris pandosia, Hewitson, Ex, Butt. i. Pier. pl. 2. fig. 14 (1853), ii. Pier. pl. 6. fig. 39 (1861).

Venezuela.

B.M.

11. Pieris pisonis.

Pieris pisonis, Hewitson, Exot. Butt. ii. Pier. pl. 6. figs. 40, 41 (1861).

E. Peru.

B.M.

12. Pieris habra.

Pieris habra, Doubleday, Ann. \& Mag. Nat. Hist. xvii. p. 22 (1846) ; Doubl. \& Hewitson, Gen. D. L. pl. 6. fig. 1 (1847).

Honduras.

B.M. 
13. Pieris locusta.

Pieris locusta, Felder, Wien. ent. Mon. v. p. 81. n. 31 (1861); Reise Nov. Lep. ii. p. 175. n. 163, pl. 25. figs. 8, 9 (“' 1865 ").

Bogotá.

14. Pieris sevata.

Pieris sevata, Felder, Wien. ent. Monat. v. p. 81 . n. 32 (1861). New Granada; Venezuela.

15. Pieris diana.

Pieris diana, Felder, Wien. ent. Mon. v. p. 81. n. 33 (1861).

New Granada.

Genus 7. Appias, Hübner.

Verz. bek. Schmett. p. 91 (1816).

1. Appias cardena.

Pieris cardena, Hewitson, Ex. Butt. ii. Pier. pl. 3. figs. 17, 18 (1861).

Var. Pieris hagar, Vollenhoven, Monogr. Pier. p. 38. n. 49, pl. 4. fig. 6 (1865̃).

Sarawak.

B.M.

2. Appias hombronit.

Pieris hombronii, Lucas, Rev. Zool. p. 325 (1852) ; Vollenhoven, Mon. Pier. p. 5. n. 2, pl. 2. fig. 3 (1৮6j).

Celebes.

\section{Appias alope.}

o. Tachyris alope, Wallace, Trans. Ent. Soc. London, 3, vol. iv. p. 572 . n. 24 (1867).

Pieris amasene, Boisduval (nec Cramer), Sp. Gén. Lép. i. p. 535.

n. 143 (1836).

․ Pieris neombo, Moore, Cat. Lep. E. I. C. i. pl. $2 a$. fig. 3 (185̃).

Java; Sumatra; Borneo.

Both sexes stand as $P$. neombo in Dr. Horsfield's collection.

B.M.

4. Appias psyche.

Pieris psyche, Felder, Reise der Novara, Lep. ii. p. 166. n. 143 (1865).

New Caledonia.

5. Appias galathea.

Pieris galathea, Felder, Verh. Zool.-bot. Ges. Wien, xii. p. 485. n. 120 (1862).

Var. Pieris galene, Felder, Reise der Novara, Lep. ii. p. 165. n. 141 (" 1865 ").

Sambelong; Ceylon.

Allied to $A$. albina. 
6. Appias ega.

๙ . Pieris ega, Boisduval, Sp. Gén. Lép. 1, p. 536 . n. 144 (1836);

Feisthamel, Rev. Zool. pl. 18. fig. 2 (1839).

․ Pieris melania, Boisduval (nec Fabricius), Sp. Gén. Lép. i. p. 537 . n. 146 (1836).

Var. Pieris melania, var. caledonica, Felder, Verh. Zool.-botan. Ges. xii. p. 495. n. 207 (1862).

Frankland Isles; New Caledonia ; Port Stephen; Clarence River ; Hunter River.

B.M.

7. Appias agave.

Pieris agave, Felder, Wien. ent. Monats. vi. p. 286. n. 44 (1862).

Pieris zoë, Vollenhoven, Monogr. Pier. p. 37. n. 48, pl. 4. fig. 5 (1865).

Tachyris jacquinotii, Wallace (nec Lucas) Trans. Ent. Soc. London, 3 , vol. iv. p. 372 . n. 23 (1867).

Philippines.

B.M.

8. Appias paulina.

ㅇ․ Papilio paulina, Cramer, Pap. Exot. ii. pl. 110. figs. E, F (1779).

Catophaga leis, Hübner, Zutr. ex. Schmett. figs. 771, 772 (1832). Java; Penang; Moulmein; Assam.

B.M.

9. Appias neombo.

ㅇ. Pieris neombo, Boisduval, Sp. Gén. Lép. i. p. 539. n. 148 (1836).

Ceylon.

B.M.

10. Appias leptis.

Pieris leptis, Felder, Reise, Nov. Lep. ii. p. 163. n. 136 ("1865”). Pieris paulina, Boisduval, Sp. Gén. Lép.i. p. 538. n. 147 (1836). Java.

B.M.

\section{Appias urania.}

Tachyris urania, Wallace, Trans. Ent. Soc. London, 3, vol. iv. p. 371. n. 22 (1867).

Celebes.

\section{Appias amarella.}

Tachyris amarella, Wallace, Trans. Ent. Soc. London, 3, vol. iv. p. 373 . n. 25 , pl. 9. fig. 2 (1867).

New Caledonia.

B.M.

13. Appias acrisa.

Pieris acrisa, Boisduval, Bull. Soc. Ent. France, p. 156 (1859).

Woodlark Island. 


\section{Appias celestina.}

o. Pieris celestina, Boisduval, Voy. de l'Astrolabe, Lép. p. 46. n. 1 (1832); Lucas, Lép. Exot. pl. 23. fig. 1 (1835); ㅇ, Wallace, Trans. Ent. Soc. London, 3, vol. iv. p. 374. n. 28, pl. 8. figs. 6, $6 a$ (1867).

Aru Islands.

B.M.

15. Appias melania.

ㅇ. Papilio melania, Fabricius, Syst. Ent. p. 475. n. 140 (1775); Butler in Brenchley's Voy. App. (pl. 4 of Lep.) figs. 4, 5, ined.

Australia.

Coll. Banks in B.M.

I think there is no doubt that this is a female Appias near A. celestina.

\section{Appias athama.}

Pieris athama, Lucas, Rev. Zool. p. 336 (1852) ; Blanchard, Voy. Pôle Sud, p. 381, pl. 1. figs. 10, 11 (1853).

New Caledonia.

B.M.

\section{Appias clementina.}

Pieris clementina, Felder, Sitzungsber. Ak. Wiss. Wien, math.nat. Cl. xl. p. 448. n. 1 (1860); Reise der Novara, Lep. ii. p. 162. n. 133, pl. 25. fig. 6 (“' 1865 ”).

Amboina ; Ceram.

18. Appias cynisca.

Tachyris cynisca, Wallace, Trans. Ent. Soc. London, 3, vol. iv. p. 375 . n. 31 (1867).

Bouru.

19. Appias eumelis.

Pieris eumelis, Boisduval, Voy. de l'Astrolabe, Lép. p. 50. n. 8 (1832).

New Ireland.

20. Appias cycinna.

ơ . Pieris cycinna, Hewitson, Ex. Butt. ii. Pier. pl. 4. figs. 23, 26, Introd. p. 4 (1861).

Pieris concinna, Hewitson, l.c. text (1861).

․ Pieris ocina, Hewitson, l.c. figs. 24, 25 (186l).

Aru.

21. Appias corinna.

Tachyris corinna, Wallace, Trans. Ent. Soc. 3, vol. iv. p. 377 . n. 35 (1867).

Waigiou.

22. Appias liberia.

Papilio liberia, Cramer, Pap. Exot. iii. pl. 210. figs. G, H (1782). Amboina. 
23. Appias eliada.

Pieris eliada, Hewitson, Ex. Butt. ii. Pier. pl. 4. figs. 27,28 (1861).

Pieris liberia, Hewitson, l. c. Introd. p. 4 (1861); Vollenhoven, Monogr. Pier. p. 45. n. 60, pl. 5. fig. 4 (1865).

Ceram.

B.M.

24. Appias placidia.

Papilio placidia, Stell, Suppl. Cramer, pl. 28. figs. 4, 4 c (1790). Ceram; Amboina.

B.M.

25. Appias fatime.

Pieris fatime, Vollenhoven, Tijd. voor Ent. 2, vol. i. p. 59, pl. 2. figs. 1, 2 (1866).

Celebes.

26. Appias nero.

o . Papilio nero, Fabricius, Ent. Syst. iii. p. 153.n. 471 (1793);

Donovan, Ins. Ind. pl. 32. fig. 1 (1800).

Pieris thyria, Godart, Enc. Méth. ix. p. 147. n. 101 (1819); Lucas, Lép. Exot. pl. 25. fig. 3 (1835).

ㅇ? Pieris figulina, Butler, Ann. \& Mag. Nat. Hist. 3, vol. xx.

p. 399 , pl. 8. fig. 1 (186 $)$.

Java; Siam; Singapore; Borneo.

B.M.

27. Appias galba.

Tachyris galba, Wallace, Trans. Ent. Soc. London, 3, vol. iv. p. 378. n. 41 (1867).

Silhet.

B.M.

28. Appias domitia.

Pieris domitia, Felder, Wien. ent. Monats. vi. p. 285. n. 41 (1862).

Philippines.

B.M.

29. Appias zarinda.

Pieris zarinda, Boisduval, Sp. Gén. Lép. i. p. 486. n. 73, pl. 18. fig. 4 (1836).

Celebes.

B.M.

30. Appias bouruensis.

Tachyris bouruensis, Wallace, Trans. Ent. Soc. London, 3, vol. iv. p. 379. n. 44 (1867).

Bouru.

31. Appias zamboanga.

Pieris zamboanga, 'Felder, Wien. ent. Monats. vi. p. 285. n. 42 (1862); Reise Nov. Lep. ii. p. 162. n. 134, pl. 24. figs. 2, 3(1865).

Philippines.

B.M. 
32. Appias asterope. Pieris asterope, Felder, Wien. ent. Monats. vi.p. 286. n. 43(1862).
Luzon.

33. Appias ithome.

o. Pieris ithome, Felder, Wien. ent. Monats. iii. p. 180. n. 2, pl. 4. fig. l (1859); + , Vollenhoven, Monogr. Pier. p. 391. n. 52,
pl. 5. fig. l (1865).

Celebes.

34. Appias nephele.

B.M.

Pieris nephele, Hewitson, Ex. Butt. ii. Pier. pl. 5. fig. 33 (1861). Philippines.

B.M.

35. Appias clavis.

Tachyris clavis, Wallace, Trans. Ent. Soc. 3, vol.iv. p. 367. n. 13 (1867).

Ké Island.

36. Appias ada.

Papilio ada, Cramer, Pap. Exot. iv. pl. 363. figs. C, D (1782). ("1865").

Aru.

37. Appias enarete.

B.M.

Pieris enarete, Boisduval, Sp. Gén. Lép. i. p. 480 . n. 61 (1836); Feisthamel, Rev. Zool. pl. 18. fig. 1 (1839).

Borneo.

B.M.

38. Appias hippo. (1782).

9. Papilio hippo, Cramer, Pap. Exot. iii. pl. 195. figs. B, C

Ceylon.

The Ceylonese specimens differ slightly from the typies from Sumatra, the hind wings of the male being the typical form beneath, and those of the female paler being more deeply coloured differences prove to be constant, the on both surfaces; if these separated.

39. Appias vacans.

․ Appias vacans, Butler, Trans. Ent. Soc. Lond. p. 490 (1870); Lep. Exot. ii. pl. 34. figs. 5, 6 (Jan. 1872).

Darjeeling.

40. Appias eleonora.

Coll. H. Roberts.

Pieris eleonora, Boisduval, Sp. Gén. Lép. i. p. 481. n. 64 (1836).

Silhet; Moulmein.

B.M. an error. 
41. Appias andrea.

๙. Colias andrea, Eschscholtz, Kotzeb. Reise, iii. p. 215, pl. 23. figs. $a, b$ (1821).

(1793).

Philippines.

B.M.

42. Appias formosana.

Pieris formosana, Wallace, Proc. Zool. Soc. p. 356 . n. 5 (1866).

43. Appias lycaste.

Pieris lycaste, Felder, Reise der Novara, Lep. ii. p. 164. n. 138 ("1865").

Celebes.

B.M.

44. Appias lyncida.

๙. Papilio lyncida, Cramer, Pap. Exot. ii. pl. 131. fig. B (1779).

Mylothris monuste, Hübner, Verz. bek. Schmett. p. 91 . n. 946 (1816).

Pieris enyo, Boisduval, Sp. Gén. Lép.i. p. 481 .n. 65 (1836). (1865).

․ Pieris hippo (part), Vollenhoven, Monogr. Pier. p. 42.n. 55

Java.

B.M.

We have specimens, possibly referable to this species, from Baly, Lombock, and Amboina; but until I have seen more examples I hesitate to consider them identical with it.

45. Appias lynceola.

Pieris lynceola, Felder, Reise der Novara, Lep. ii. p. 164. n. 137 (" 1865 ").

Timor.

A local form of $A$. lyncida.

46. Appias abnormis.

Tachyris abnormis, Wallace, Trans. Ent. Soc. London, 3, vol. iv. p. 368.n. 14, pl. 8. fig. 5 (1867).

Papua.

47. Appias panthea.

Tachyris panthea, Wallace, Trans. Ent. Soc. London, 3, vol. iv. p. $376 . \mathrm{n} .33$ (1867).

Philippines.

B.M.

48. Appias panda.

Pieris panda, Godart, Enc. Méth.ix.p. 147.n. 102 (1819).

Pieris sulphurea, Vollenhoven, Monogr. Pier. p. 32. n. 41, pl. 4. fig. 4 (1865).

Java.

B.M. 
49. Appias albina.

§. Pieris albina, Boisduval, Sp. Gén. Lép. i. p. 480. n. 62 (1836).

Pieris nathalia, Felder, Wien. ent. Monats. vi. p. 285. n. 40 (1862).

Philippines; Celebes.

B.M.

50. Appias darada.

Pieris darada, Felder, Reise der Novara, Lep. ii. p. 166. n. 142 ("1865").

Moulmein.

B.M.

5̃. Appias libythea.

ㅇ․ Papilio libythea, Fabricius, Syst. Ent. p. 471 .n. 120 (1775).

Papilio zelmira, Cramer, Pap. Exot.iv. pl. 320. figs. E, F (1782).

Papilio libithea (sic), Herbst, Natursyst. Schmett. pt. 5, Index.

Pieris libitina, Godart, Enc. Méth. ix.p. 133. n. 44 (1819).

Pieris nerissa, Godart, Enc. Méth. ix. p. 142. n. 84 (1819).

๙. Pieris rouxii, Boisduval, Sp. Gén. Lép. i. p. 481. n. 63 (1836).

Pieris jacquinotii, Lucas, Rev. Zool. p. 326 (1852).

Punjaub ; Barrackpore; Ceylon.

B.M.

52. Appias Zelmira.

Papilio zelmira, Cramer, Pap. Exot. iv. pl. 320. figs. C, D (1782).

? Pieris larissa, Felder, Reise der Novara, Lep. ii. p. 166. n. 144 ("1865").

Silhet; Moulmein ; ? E. Indies.

B.M.

53. Appias saba.

Papilio saba, Fabricius, Sp. Ins. ii.p. 46. n. 199 (1781).

Papilio epaphia, Cramer, Pap. Exot. iii. pl. 207. figs. D, E (1782).

Papilio hypatia, Drury, Ill. Ex. Ent. iii. pl. 32. figs. 5, 6 (1782).

Pieris higinia, Godart, Enc. Méth. ix. p. 133. n. 45 (1819).

Pieris malatha, Boisduval, Faun. de Madag. p. 18. n. 4, pl. 1. figs. 4, 5 (1833).

? Papilio nigricantemarginatus, Goeze, Beytr. i. p. 184. и. 95 (1779).

Ashanti ; Sierra Leone; Port Natal.

B.M.

54. Appias poeyi, sp. nov.

ㅇ. Pieris ilaire, Poey (nec Godart), Cent. Lep. (1833).

St. Domingo ; Panamá; Honduras.

B.M.

The species figured by Poey has nothing to do with the Brazilian A. ilaire, although much like it in the male sex.

5.j. Appias illaire.

๙. Pieris ilaire, Godart, Enc. Méth.ix. p. 142. n. 83 (1819).

Papilio albunea, Dalman, Anal. Ent. p. 39 (1823).

Brazil.

B.M.

Proc. Zool. Soc.-1872, No. IV. 
56. Appias margarita.

Mylothris margarita, Hübner, Samml. ex. Schmett. (1816-41).

Panamá.

B.M.

Nearly allied to $A$. ilaire.

57. Appias drusilla.

Papilio drusilla, Cramer, Pap. Exot. ii. pl. 207. fig. C (1779). Honduras.

B.M.

58. Appias castalia.

๙. Papilio castalia, Fabricius, Ent. Syst. iii. p. 188. n. 580 (1793); Donovan, Ins. Ind. pl. 28. fig. 3 (1800).

ㅇ․ Pieris mysia, Godart, Enc. Méth.ix. p. 143. n. 87 (1819).

ㅇ․ ? Papilio fuscofimbriatus, Goeze, Beytr.p. 182.n. 81 (1779). Brazil.

B.M.

59. Appias molpodia.

Mylothris molpodia, Hübner, Zutr. ex. Schmett. figs. 259, 260 (]823).

Jamaica.

B.M.

60. Appias pandione.

Hiposcritia pandione, Hübner, Zutr. ex. Schmett. figs. 651, 652 (1832).

Pieris philonome, Lucas (nec Boisduval), Rev. Zool. p. 334 (1852).

Pieris paulina, var., Vollenhoven, Monogr. Pier. p. 33. n. 43 (1865).

Java.

B.M.

61. Appias lucasir.

Tachyris lucasii, Wallace, Trans. Ent. Soc. London, 3, vol. iv. p. 381. n. 50 (1867).

Pieris pandione, ơ, Boisduval, Sp. Géu. Lép. i. p. 537. n. 145 (1836).

Java.

Intermediate between $A$. indra and $A$. durvasa (Lalage, ơ var.).

62. Appias indra.

Pieris indra, Moore, Proc. Zool. Soc. p. 103, pl. 44. fig. 5 (1857).

Silhet; Darjeeling; Calcutta.

B.M.

63. Appias lalage.

Pieris lalage, Doubleday in Gray's Zool. Miscell. p. 76 (1842); Gen. Diurn. Lepid. pl، 6. fig. 5 (1847).

Var. ? Pieris ida, Lucas, Rev. Zool. p. 335 (1852).

Pieris durvasa, Moore, Proc. Zool. Soc. p. 103, pl. 44. fig. 6 (1857).

Silhet, Assam, Darjeeling, Nepal, E. India.

B.M. 
64. Appias phebe.

Pieris phobe, Felder, Wien. ent. Monats. v. p. 299. n. 7 (1861); Reise der Novara, Lep. ii. p. 163. n. 135, pl. 25. fig. 5 (1865).

Luzon.

65. Appias zamora.

․ Pieris zamora, Felder, Wien. ent. Monats. vi. p. 286. n. 45 (1862).

Philippines.

Closely allied to $A$. phoebe.

66. Appias albomaculatus.

Papilio albomaculatus, Goeze, Beytr. i. p. 182. n. 79 (1779); Seba, Thes. iv. pl. 13. figs. 7, 8.

Genus 8. Phrissura, Butler,

Cist. Ent. iii. pp. 37, 49 (1870); Tr. Ent. Soc. Lond. p. 171 (1871).

1. Phrissura polisma.

o. Pieris polisma, Hewitson, Ex. Butt. ii. Pier. pl. 6. fig. 38 (1861); +, iii. Pier. pl. 8. fig. 55 (1866).

Celebes.

2. Phrissura egis.

․ Pieris agis, Felder, Wien. ent. Monats. v. p. 299. n. 6 (1861); Reise der Novara, Lep. ii. p. 175. n. 162, pl. 24. fig. 1 (“1865”). (1862).

. Pieris illana, Felder, Wien. ent. Monats. vi. p. 287. n. 48

Philippines.

B.M.

Genus 9. Daptonura*, Butler,

Cat. Fabr. Diurn. Lep. p. 209 (1869).

1. Daptonura lycimnia.

Papilio lycimnia, Cramer, Pap. Exot. ii. pl. 105. figs. E, F (1779).

Papilio fippantha, Fabricius, Ent. Syst. 3, i. p. 202. n. 631 (1797).

Mylothris agrippina, Hübner, Verz. bek. Schmett. p. 91 . n. 949 (1816).

Pará; Brazil.

B.M.

2. Daptonura polyhymia.

Pieris polyhymnia, Felder, Reise der Novara, Lep. ii. p. 170. n. 152 (“1865”).

Bogota.

Perhaps only a yellow variety of $D$. Iycimnia.

B.M.

* This should be the spelling of the name; T originally, by sheer inadvertence, inserted the $o$ as in the Greek original. 


\section{Daptonura eUrymia.}

๙. Pieris eurymnia, Felder, Reise der Novara, Lep. ii. p. 170. n. 153 (" 1865 ").

Venezuela.

B.M.

\section{Daptonura salacia.}

Pieris salacia, Godart, Enc. Méth. ix. p. 144. n. 91 (1819).

Pieris vecticlusa, Butler, Proc. Zool. Soc. London, p. 456. n. 3, pl. 26. fig. 3 (1865).

Cuba.

B.M.

\section{Daptonura limnoria.}

Pieris limnoria, Godart, Enc. Méth. ix. p. 144. n. 93 (1819); (Mylothris, L.) Hübner, Samml. exot. Schmett. iii. (181 (;-41).

Melete limnobia, Swainson, Zool. Ill. ii. pl. 79 (1833).

Brazil.

B.M.

6. Daptonura pantoporia.

Mylothris pantoporia, Hübner, Samml. ex. Schmett. iii.(1816-41). ?

B.M.

7. Daptonura eubotea.

Pieris eubotea, Godart, Enc. Méth. ix. p. 144. n. 90 (1819).

? Papilio cano-exalbidus, Goeze, Beytr. i. p. 183. n. 85 (1779).

Peruvian Amazons.

B.M.

8. Daptonura elia.

Pieris alia, Felder, Wien. ent. Monats. v. p. 82 . n. 34 (1861).

Var.? Pieris laria, Felder, Reise der Novara, Lep. ii. p. 171. n. 155 ("1865").

Bolivia.

B.M.

9. Daptonura leucadia.

Euterpe leucadia, Felder, Wien. ent. Monats. vi. p. 67.n. 8(1862).

Rio Negro.

Closely allied to $D$. alia.

10. Daptonura peruviana.

Pieris peruviana, Lucas, Rev. Zool. p. 327 (1852).

Id.? E. Peru.

B.M.

\section{Daptonura leucanthe.}

Pieris leucanthe, Felder, Wien. ent. Monats. v. p. 82 .n. 35 (1861). Archidona; Cuenca ; E. Peru.

B.M.

Possibly a variation of $D$. peruviana.

12. Daptonura isandra.

Pieris isandra, Boisduval, Sp. Gén. Lép. i. p. 490. n. 79 (1836). Brazil; Polochic valley, Nicaragua ; Jamaica. 
13. Daptonura? evonima. Pieris evonima, Boisduval. Sp. Gén. Lép. i. p. 493. n. 84 (1836).
Cuba.

Genus 10. Belenois, Hübner,

Verz. bek. Schmett. p. 92 (1816).

\section{Belenois calypso.}

Papilio calypso, Drury, Ill. Ex. Ent. ii. pl. 17. figs. 3, 4 (1773).

Papilio nigronotatus, Goeze, Beytr. i. p. 182. n. 76 (1779).

Papilio retorta, Goeze, Beytr. i. p. 182. n. 78 (1779).

Papilio nigropictus, Goeze, Beytr. i. p. 183 . n. 83 (1779).

Sierra Leone; Ashanti ; Congo.

B.M.

2. Belenois sabina.

Pieris sabina, Felder, Reise Nov. Lep. ii. p. 167. n. 145 (“1865"). Sierra Leone.

Like a white $B$. ianthe on the upper surface.

B.M.

3. Belenois ianthe.

Pieris ianthe, E. Doubleday, Gray's Zool. Misc. p. 77 (1842).

Belenois ianthe, Butler, Lep. Exot. ii. pl. 34. fig. 8 (1872).

Sierra Leone.

B.M.

4. Belenois rhena. (1846).

Pieris rhena, Doubleday, Ann. \& Mag. Nat. Hist. xvii. p. 24 o, Ashanti; + , W. Africa.

B.M.

5. Belenois hedyle.

Papilio hedyle, Cramer, Pap. Exot. ii. pl. 186. figs. C, D (1779).

? Papilio sordide-flavicans, Goeze, Beytr. i. p. 182 . n. 82 (1779). Ashanti.

B.M.

6. Belenois theora.

Pieris theora, E. Doubleday, Ann. \& Mag. Nat. Hist. xvii. p. 25 (1846) ; Doubl. \& Hew. Gen. Diurn. Lepid. pl. 6. fig. 4 (1847). (" o var.? 1865 ").

0 , Niger; $q$, Ashanti.

B.M.

7. Belenois helcida.

Pieris helcida, Boisduval, Faun. de Madag. p. 17. n. 1, pl. 2. figs. 1, 2 (1833).

Madagascar.

8. Belenois thysa.

Pieris thysa, Hopffer, Ber. Verh. Ak. Berl. p. 639. n. 1 (1855);

Peters's Reise, Zool. v. p. 349, pl. 21. figs. 7-10 (1862). 
Pieris sabrata, E. Doubleday, Gen. Diurn. Lepid. p. 47. n. 62 (1847).

Pieris agathina, var. A., Trimen, Rhop. Ap. Austr. p. 29 (1862). Congo.

B.M.

9. Belenois meldole, Butler, Proc. Zool. Soc. (1871).

Loanda.

Coll. R. Meldola.

10. Belenois matuta.

Pieris matuta, E. Doubleday, Ann. \& Mag. Nat. Hist. xx. p. 64 (1847).

Sierra Leone; Fernando Po.

B.M.

11. Belenois larima.

Pieris larima, Boisduval, Sp. Gén. Lép. i. p. 524. n. 126 (1836). Congo.

B.M.

12. Belenois Pigea.

․ Pieris pigea, Boisduval, Sp. Gén. Lép. i. p. 523. n. 124 (1836).

o. Pinacopteryx alba, Wallengren, Lep. Rhop. Caffr. p. 10 (1857).

Belenois inana, Butler, Trans. Ent. Soc. London, p. 526. n. 1 (1870).

Zulu ; Port Natal.

$\delta^{*}$, ㅇ, B.M.

13. Belenois orbona.

Mylothris orbona, Hübner, Zutr. ex. Schmett. figs. 985, 986 (1832). Africa.

B.M.

14. Belenois simana.

Pieris simana, Hopffer, Ber. Verh. Ak. Berl. p. 640. n. 13 (1855); Peters's Reise n. Mossamb. Zool. v. p. 354, pl. 23. figs. 3-6 (1862). S. Africa.

B.M.

\section{Belenois Charina.}

Pieris charina, Boisduval, Sp. Gén. Lep. i. p. 525. n. 128 (1836). ․ Pieris anactorie, E. Doubleday, Gray's Zool. Miscell. p. 77 (1842).

‥ Papilio nelo, Bergsträsser, pl. 32. fig. 2 ; Herbst, Natursyst. Schmett. pl. 87. fig. 9 .

Knysna ; Port Natal ; Zulu country,

B.M.

As Mr. Doubleday hardly ever labelled his types, some of them (amongst which is that of $P$. anactorie) seem to have been turned out of the Collection as worn-out specimens; at any rate, they do not now exist there.

16. Belenois phaola.

Pieris phaola, E. Doubleday, Ann. \& Mag. Nat. Hist. xx. p. 63 (1847).

Dahomey. 
17. Belenois hyoma.

Pieris hyoma, Boisduval. Sp. Gén. Lép. i. p. 508. n. 102 (1836). E. Africa.

18. Belenots confusa, sp. n.

Pieris phileris, $q$, Boisduval, Faun. Lép. de Madag. pl. 2. fig. 5 (1833).

Madagascar.

19. Belenois eudoxia.

Papilio eudoxia, Drury, Ill. Ex. Ent. iii. pl. 32. figs. 1, 2 (1782). Ashanti.

B.M.

20. Belenois sylvia.

Papilio sylvia, Fabricius, Syst. Ent. p. 470 . n. 115 (1775).

Papilio sylphia, Herbst, Natursyst. Schmett. v. p. 47. n. 21.

Sierra Leone.

B.M.

21. Belenois Cynis.

Pieris cynis, Hewitson, Ex. Butt. iii. Pier. pl. 8. fig. 54 (1866).

Malacca.

B.M.

\section{Belenois zochalia.}

Pieris zochalia, Boisduval, Sp. Gén. Lép. i. p. 508. n. 100 (1832).

S. Africa ; Knysna, Cape of Good Hope.

B.M.

23. Belenois gidica.

Pieris gidica, Godart, Enc. Méth. ix. p. 131. n. 37 (1819).

Pinacopteryx doubledayii et westwoodii, Wallengren, Lep. Rhop. Caffr. pp. 8, 9 (1853).

Port Natal; Plettenberg Bay; Zulu Country; Knysna. B.M.

24. Belenois mesentina.

Papilio mesentina, Cramer, Pap. Exot. iii. pl. 270. figs. A, B (1782).

Papilio aurota, Fabricius, Ent. Syst. iii. p. 197. n. 614 (1793).

Punjaub; Barrackpore; Bengal; Benares; Ceylon.

B.M.

25. Berenois augusta.

Papilio angusta, Olivier, Voy. dans l'Emp. Ott. Atlas, pl. 33. figs. $3 a, b$ (1801).

Asia Minor.

Possibly a variety of $B$. mesentina.

26. Belenois teutonia.

Papilio teutonia, Fabricius, Syst. Ent. p. 474. n. 137 (1775); Sulzer, Gesch. Ins. pl. 15. fig. 9 (1776).

New Holland; Queensland.

B.M. 
27. Belenois clytie.

Papilio clytie, Donovan, Ins. New Holland, pl. 19. fig. 2 (1805). Papilio coronea, 우, Herbst, Natursyst. Schmett. pl. 98. fig. 89. N. Australia.

B.M.

28. Belenois niscia.

Pieris niscia, $\mathrm{M}^{\mathrm{c}}$ Leay, in King's Surv. Austr. ii. App. p. 459. n. 138 (1827).

New Holland; West Australia.

B.M.

29. Belenois Jave.

Papilio java, Sparrmann, Amœn. Acad. vii. p. 504. n. 1 (1767). Papilio coronea, Cramer, Pap. Exot. i. pl. 68. figs. B, C (1779). Papilio deiopeia, Donovan, Ins. New Holl. pl. 21 . fig. 2 (1805). Java; Bali ; Flores ; Amboina.

B.M.

30. Belenois PERICTIONE.

Pieris perictione, Felder, Reise Nov. Lep. ii. p. 168. n. 149 ("1865").

Aru Islands.

31. Belenois creona.

Papilio creona, Cramer, Pap. Exot. i. pl. 95. figs. C-F (1779).

-Var. Papilio helcita* (part), Fabricius, Syst. Ent. p. 475. n. 141 (1775).

Papilio cneora, Fabricius, Ent. Syst. iii. p. 191. n. 594 (1797). Colias ernestius, Laporte, Bull. Soc. Linn. Bord. iv. (18-?).

Senegal.

B.M.

Var. Papilio severina, Cramer, Pap. Exot. iv. pl. 338. figs. G, H (1782).

Pieris agrippina, Felder, Reise Nov. Lep. ii. p. 173. n. 159 (1865). Port Natal; Zulu country.

B.M.

Var. Pieris boguensis, Felder, Reise Nov. Lep. ii. p. 173. n. 160 (1865).

Pieris elisa, Vollenhoven, in Pollen \& Van Damm's Faun. Madag. v. p. 12. n. 9, pl. 2. fig. 3 (1869).

White Nile.

B.M.

32. Belenois Syrinx.

Pinacoptery $x$ syrinx, Wallengren, Wien. ent. Mon. iv. p. 34. n. 4 (1860).

S. Africa.

Belongs to the Creona group.

33. Belenois scyllaria.

Pieris scyllaria, McLeay, King's Surv. Austr. ii. App. p. 459. n. 139 (1827).

Pieris lanassa, Boisduval, Sp. Gén. Lép. i. p. 477. n. 57 (1836). * Also of Goeze's Beyträge, i. p. 147. n. 94 (1779). 
Var. Pieris nabis, Lneas, Rev. Zool. p. 326 (1852).

Pieris perithea, Felder, Reise Nov. Lep. ii. p. 169.n. 150 (1865). N. Australia ; Port Essington; Cape York.

B.M.

\section{Belenots periclea.}

Pieris periclea, Felder Reise Nov. Lep. ii. p. 169.n. 151 ("1865"); Herrich-Scbäffer, Stett. ent. Zeit. p. 76. n. 45, pl. 1. fig. 4 (1869). Aneiteum, New Hebrides; N.W. Australia.

B.M.

35. Belenois perimale.

Papilio perimale, Donovan, Ins. New Holl. pl. 20. fig. 1 (1805). Var.? Pieris narses, Wallace, Trans. Ent. Soc. London, 3, vol. iv. p. 333 . n. 13, pl. 6 . fig. 3 (1867).

Australia.

36. Belenois boisduvaliana.

Pieris boisduvaliana, Felder, Wien. ent. Mon. vi. p. 287. n. 47 (1862); Reise Nov. Lep. ii. p. 168. n. 147, pl. 24. fig. 8 ("1865").

Pieris nephele, ㅇ, Felder, Wien. ent. Mon. v. p. 299. n. 8 (1861). Manilla.

B.M.

37. Belenois rachel.

Pieris rachel, Boisduval, Sp. Gén. Lép. i. p. 469. n. 46 (1836).

Pieris wallaceana, Felder, Reise Nov. Lep.ii. p. 168.n. 148 (1865).

Waigiou.

B.M.

38. Belenois peristhene.

Pieris peristhene, Boisduval, Bull. Soc. Ent. France, p. 155. n. 4 (1859).

New Caledonia, New Ireland, and Aneiteum (New Hebrides). B.M.

39. Belenois pitys.

Pieris pitys, Godart, Enc. Méth. ix. p. 134. n. 48 (1819); Lucas, Lep. Ex. pl. 29. fig. 1 (1835).

Java ; Timor.

B.M.

40. Belenois mentes.

Pieris mentes, Wallace, Trans. Ent. Soc. London, 3, vol. iv. p. 332.

n. 11 (1867).

Lombock.

B.M.

41. Belenois afFinis.

Pieris "ffinis, Vollenhoven, Mon. Pier. p. 40. n. 53. pl. 5. fig. 2
(865).

Celebes.

B.M.

42. Belenois? erastus.

Pieris erastus, Hewitson, Ex. Butt. iii. Pier. pl. 8. fig. 51 (1866).
Gaboon. 
43. Belenois? Abyssinica.

Pieris abyssinica, Lucas, Rev. Zool. p. 328 (1852).

Abyssinia.

Genus 11. Pontia, Fabricius,

Illiger's Mag. vi. p. 283 (1807).

\section{Pontia crategi.}

Papilio cratagi, Linnæus, Faun. Suec. p. 269. n. 1034 (1761).

Papilio nigronervosus, Retz. Gen. Spec. Ins. p. 30. n. 2 (1783).

England; France; Germany; Syria; N. Japan. B.M.

\section{Pontia hippià.}

Pieris hippia, Bremer, Bull. Acad. Pét. iii. p. 464 (1861); Lep. Ost-Sibir. p. 7. n. 12, pl. 3. fig. I (1864).

Leuconia cratcegi, var. cratagoides, Lucas, Ann. Soc. Ent. France, p. 503, pl. 11. fig. 11 (1866).

China.

3. Pontia soracta.

Aporia soracta, Moore, Cat. Lep. East-Ind. Comp. 1, p. 83. n. 170 (1857).

Himalayas.

B.M.

\section{Pontia menapia.}

Pieris menapia, Felder, Wien. ent. Mon. iii. p. 27 ]. n. 18 (1859); Reise der Nov. Lep. ii. p. 181. n. 172, pl. 25. fig. 7 ("1865").

Pieris tau, Scudder, Proc. Boston Nat. Hist. Soc.viii. p.183(1861).

Pieris ninonia, Boisduval, Lép. Cal. p. 38 . n. 5 (1869).

British Colombia, West Coast of America.

B.M.

5. Pontia terlooir.

Neophasia terlooii, Behr, Trans. Am. Ent. Soc. p. 304 (1869).

California.

\section{Pontia notha.}

Euterpe notha, Lucas in Guérin's Rev. Zool. p. 195 (1852).

Venezuela.

B.M.

7. Pontia pieridoides.

Euterpe pieridoides, Felder, Reise der Novara, ii. p. 158. n. 126 ("1865").

Bogotá (Lindig), Felder.

"In marking and coloration, especially on the underside of the hind wings, this species strikingly calls to mind Pieris locusta, Felder, from Bogotá."

\section{Pontia corcyra.}

Euterpe corcyra, Felder, W. e. Monatschr. iii. p. 327 (1859); Reise der Novara, ii. p. 159. n. 127, pl. 23. fig. 8 (“1865"). E. Peru. 
9. Pontia calymina.

Euterpe calymnia, Felder, Wien. ent. Monats. vi. p. 67. n. 7 (1862); Reise der Novara, Lep. ii. p. 171. n. 154, pl. 23 . fig. 7 ("1865").

Rio Negro.

10. Pontia remba.

Pieris remba, Moore, Cat. Lep. E. I. C. i. p. 75. n. 147 (1857).

Var. Pieris amba, Wallace, Trans. Ent. Soc. London, 3, vol. iv. p. 340 . n. 36 (1867).

N. India; Canara; Ceylon. B.M.

11. Pontia nadina.

Pieris nadina, Lucas, Rev. Zool. p. 333 (1852).

Id. var.? Pieris nama, Moore, Cat. Lep. Mus. E. I. C. i. p. 76. n. 148 (1857); Proc. Zool. Soc. p. 102, pl. 44. figs. 1, 2 (1857).

Darjeeling; Silhet; N. Australia.

B.M.

12. Pontia eperia.

Pieris eperia, Boisduval, Sp. Gén. Lép. i. p. 470 . n. 48 (1836).

Celebes.

B.M.

13. Pontia nerissa.

Papilio nerissa, Fabricius, Syst. Ent. p. 471 . n. 123 (1775).

Papilio amasene, Cramer, Pap. Exot. i. pl. 44. fig. A (1776).

Papilio coronis, Cramer, Pap. Exot. i. pl. 44. figs. B, C (1776).

Papilio coronnis (sic), Herbst, Natursyst. Schmett. v. p. 88. n. 25.

Hong-Kong; Moulmein; Nepal.

B.M.

14. Pontia corva. Pieris corva, Wallace, Trans. Ent. Soc. London, 3, vol. iv. p. 339.
n. 32 (1867).

Pieris coronis, var. A, Boisduval, Sp. Gén. Lép. i. p. 472 (1836). Java; Sumatra; Baly Island.

15. Pontia phryne.

B.M.

Papilio phryne, Fabricius, Syst. Ent.p. 473. n. 131 (1775).

Papilio evagete, Cramer, Pap. Exot. iii. pl. 221. figs. F, G (1782). Canara; Landoor; Nepal.

B.M.

16. Pontia zeuxippe.

Papilio zeuxippe, Cramer, Pap. Exot. iv. pl. 362. figs. E, F (1782).

Papilio cassida, Fabricius, Ent. Syst. Suppl. p. 427 (1798).

Pieris hira, Moore, Proc. Zool. Soc. p. 490. n. 22, pl. 31. fig. 17 (1865).

Var. Pieris copia, Wallace, Trans. Ent. Soc. 3, vol. iv. p. 340.
35 (1867).

E. India, Bombay; Malwa, N. India.

B.M. 
17. Pontia temena.

Pieris temena, Hewitson, Ex. Butt. ii. Pier. pl. 3. fig. 19 (1861). Lombock.

18. Pontia tamar.

Pieris tamar, Wallace, Trans. Ent. Soc. London, 3, vol. iv. p. 337. n. 25 , pl. 6. fig. 2 (1867).

Baly Island.

Near P. temena.

19. Pontia timnatha.

Pieris timnatha, Hewitson, Ex. Butt. iii. Pier. pl. 7. figs. 47, 48 (1862).

Tondano, Celebes.

B.M.

20. Pontia leta.

Pieris lata, Hewitson, Ex. Butt. iii. Pier. pl. 7. figs. 45, 46 (1862). Timor.

21. Pontia pactolica.

Pieris pactolicus, Butler, Proc. Zool. Soc. p. 455. n. 1, pl. 26. fig. 1 (1865).

Borneo.

B.M.

22. Pontia clemanthe.

Pieris clemanthe, Doubleday \& Hewitson, Gen. Diurn. Lepid. pl. 6. fig. 3 (1847).

Siam.

B.M.

23. Pontia lea.

Pieris lea, E. Doubleday, Ann. \& Mag. Nat. Hist. xvii. p. 23 (1846).

? Pieris amalia +, Vollenhoven, Mon. Pier. p. 23 (1865).

Sarawak.

B.M.

24. Pontia amalia.

Pieris amalia, Vollenhoven, Mon. Pier. p. 23. n. 28, pl. 3. fig. 6 (1865).

Singapore; Moulmein.

B.M.

25. Pontia licea.

Papilio licea, Fabricius, Mant. Ins. ii. p. 20. n. 210 (1787).

East Indies?

Seems to come near $P$. amalia.

26. Pontia jael.

Pieris jael, Wallace, Trans. Ent. Soc. London, 3, vol. iv. p. 335. n. $20(1867)$.

Bouru. 
27. Pontia naomi.

Pieris naomi, Wallace, Trans. Ent. Soc. London, 3, vol. iv. p. 336.

n. 23 (1867).

Lombock.

B.M.

28. Pontia hester.

Pieris hester, Vollenhoven, Mon. Pier. p. 24. n. 29, pl. 4. fig. 1 (1865).

Papua.

29. Pontia judith.

Papilio judith, Fabricius, Mant. Ins. ii. p. 22. n. 230 (1787) ; Donovan, Ins. Ind. pl. 27. fig. 2 (1800).

Java.

B.M.

30. Pontia olga.

Pontia olga, Eschscholtz, Kotzeb. Reise, iii. p. 214, pl. 9. figs. $21 a, 21 b(1821)$.

Philippines; Hong-Kong.

B.M.

31. Pontia aspasia.

Papilio aspasia, Stoll, Suppl. Cramer's Pap. Ex. pl. 33. figs. 3, 3 c (1790).

Pieris asterope, Godart, Enc. Méth. ix. p. 154 . n. 125 (1819).

Amboina ; Ceram.

B.M.

32. Pontia emma.

Pieris emma, Vollenhoven, Mon. Pier. p. 24. n. 30, pl. 4. fig. 2 (1865).

Var. Philippine islands.

Nearly allied to $P$. aspasia.

B.M.

Genus 12. Metaporia, Butler.

Cist. Ent. iii. pp. $38 \& 51$ (1870).

\section{Metaporia phryxe.}

Pieris phryxe, Boisduval, Sp. Gén. Lép. i. p. 446. n. 12 (1836); Blanchard in Jacquem. Voy. Inde, iv. Ins. p. 16. n. 6, pl. 2. fig. 1 (1844).

N. India.

B.M.

2. Metaporia agathon.

Pieris agathon, G. R. Gray, Zool. Misc. p. 33 (1831); Lep. Ins. Nepal, p. 8, pl. 8. fig. 1 (1846).

Nepal; N. India.

B.M.

3. Metaporia nabellica.

Pieris nabellica, Boisduval, Sp. Gén. Lép. i. p. 509. n. 104 (1836).

Himalayas. 
Genus 13. Synchlö̈, Hübner.

Verz. bek. Schmett. p. 94 (1816).

1. SYNCHLOË DAPLIDICE.

Papilio daplidice, Linnæus, Syst. Nat. i. p. 760 . n. 77 (1766); Hübner, Eur. Schm. i. figs. 414, 415 (1798-1803).

Papilio edusa, Fabricius, Gen. Ins. p. 255 (1777).

Var. Papilio bellidice, Brahm, Ill. Mag. iv. p. 362 (1805).

Papilio belemida, Hübner, Eur. Schm. i. fig. 931-4 (1827-1841).

Var. Papilio raphani (part), Esper, Eur. Schm. I. ii. pl. 84. fig. 3 (1783).

Germany; Sicily; Spain; Andalusia; Cashmere; Syria. B.M.

2. Synchlö̈ GLAUCONOME.

Pontia glauconome, Klug, Symb. Phys. pl. 7. figs. 18, 19 (1829). Arabia ; Egypt.

Allied to S. daplidice.

3. Synchloë Callidice.

Papilio callidice, Esper, Eur. Schm. I. ii. pl. 115. figs. 2, 3 (1777).

Pieris kalora, Moore, Proc. Zool. Soc. p. 489. n. 18, pl. 31. fig. 15 (1865).

Var. Pieris chrysidice, Herrich-Schäffer, Eur. Schm. i. figs. 200203 (1844).

Europe.

B.M.

4. Synchlö̈ PRotodice.

Pieris protodice, Boisduval \& Leconte, Lép. Am. Sept. p. 45, pl. 17. figs. 1-3 (1833).

Ohio ; British Columbia; Mexico.

B.M.

5. Synchloë hellica.

Papilio hellica, Linnæus, Syst. Nat. 1. ii. p. 760. n. 71 (1766).

Papilio helice, Linn. Mus. Ulr. p. 243 (1764).

Papilio daplidice, Cramer, Pap. Exot. ii.pl.171. figs. C, D (1779).

Papilio raphani, Esper, Eur. Schm. I. ii. pl. 123. figs. 3, 4 (1806?).

Cape of Good Hope; Knysna; Interior of S. Africa. B.M.

6. Synchlö Chloridice.

Papilio chloridice, Hübner, Eur. Schm.i. figs. 712, 713 (1803-18).

Papilio daplidice, Esper, Eur. Schm. I. ii. pl. 90. fig. 1 (1784).

Russia.

B.M.

7. Sychlö? Leucodice.

Pontia leucodice, Eversmann, Bull. Mosc. p. 541, pl. 7. figs. $2 a, b$ (1843).

Siberia.

Somewhat resembles the species of the genus Tatochila. 
8. SynchloË SisymbriI.

Pieris sisymbrii, Boisduval, Ann. Soc. Ent. France, p. 284 (1852). California.

Links the Napi and Daplidice groups.

9. Synchlö? CANaCe.

Pieris canace, Edwards, Trans. Am. Ent. Soc. (1870).

California.

10. Synchlö̈ hULDA.

Pieris hulda, Edwards, Trans. Am. Ent. Soc. ii. p. 370 (1870). Kodiak.

\section{SynchloË AJaka.}

Pieris ajaka, Moore, Proc. Zool. Soc. p. 490. n. 21, pl. 31. fig. 16 (1865).

Kunawur (Lang).

B.M.

12. SynchloË NAPI.

Papilio napi, Linnæus, Faun. Suec. p. 271. n. 1037 (1761).

Var. Papilio bryonia, Ochsenheimer, Eur. Schm. i. ii. p. 151 (1808).

Papilio napi, Esper, Eur. Schm. I. ii. pl. 64. figs. 3-5 (1783).

Var. Papilio ${ }^{\circ}$ napaee, Esper, Eur. Schm. I. ii. pl. 116. fig. 5 (1800?).

Var. Pontia sabellica, Steph. Ill. Haust. i. p. 21, pl. 3. figs. 3, 4 (1827).

Austria ; Germany; Lapland.

B.M.

13. Synchlö̈ Nasturtil.

Pieris nasturtii, Edwards, Proc. Ent. Soc. Philad. ii.p.501 (1864). California.

14. SynchloË virginiensis.

Pieris virginiensis, Edwards, Trans. Am. Ent. Soc. p. 13 (1870). United States.

15. Synchlö̈ oleracea.

Pontia oleracea, Harris, New Engl. Farmer, viii. p. 402 (1829).

Var. Pieris cruciferarum, Boisduval, Sp. Gén. Lép. i. p. 519. n. 119 (1836).

Var. Pontia casta, Kirby, Faun. Bor. Am. iv. p. 288, pl. 3. fig. 1 (1837).

Newfoundland; Nova Scotia ; Hudson's Bay ; Vancouver Island ; Canada West.

B.M.

Var. S. cruciferarum. Canada West.

B.M. 
16. SynChloË Frigida.

Pieris frigida, Scudder, Proc. Bost. Nat. Hist. Soc. viii. p. 181 (1861).

Pieris oleracea (part), Boisduval, Sp. Gén. Lép. i. p. 518. n. 117 (1836).

Labrador.

17. Synchlö́ venosa.

Pieris venosa, Scudder, Proc. Bost. Nat. Hist. Soc. viii. p. 182 (1861).

Pieris nasturtii, Boisduval, Lép. Cal. p. 38. n. 7 (1869).

California.

18. SynchloË Marginalis.

Pieris marginalis, Scudder, Proc. Bost. Nat. Hist. Soc. viii. p. 183 (1861).

California.

19. Synchloë Pallida.

Pieris pallida, Scudder, Proc. Bost. Nat. Hist. Soc. viii. p. 183 (186l).

Pieris yreka, Reakirt, Proc. Acad. Nat. Sci. Philad. p. 238. n. 1 (1866).

Pieris iberidis, Boisduval, Lép. Cal. p. 39 . n. 9 (1869).

California.

20. Synchlö̈ occidentalis.

Pieris occidentalis, Reakirt, Proc. Ent. Soc. Philad. vi. p. 133 (180̋6).

Colorado.

21. SynchloË vernalis.

Pieris vernalis, Edwards, Proc. Ent. Soc. Philad. ii. p. 105 (1864).

New Jersey.

22. Synchloë melete.

Pieris melete, Ménétriés, Cat. Mus. Petr. Lep. ii. p. 113, pl. 10. figs. 1, 2 (1857).

Japan.

B.M.

23. SyCNHLOË BRASSICOIDES.

Pieris brassicoides, Guérin, Lef. Voy. Abyss. vi. p. 365, pl. 9. figs. 3-6 (1849).

Abyssinia.

B.M.

We received this under the name of P. kafta; but I cannot discover that it has been anywhere described under this name. 
24. SyNCHLOË CHEIRANTHI.

Catophaya cheiranthi, Hübner, Samml. ex. Schm. ii. pl. 124 (1816-36).

Teneriffe.

B.M.

25. SynchloË nepalensis.

Pieris brassica, var. nepalensis, Gray, Lep. Ins. Nepal. pl. 6 . figs. ], 3 (1846).

Punjaub; Bhotan ; E. India; Madeira.

B.M.

26. SynchloË BRASSICA.

Papilio brassica, Linnæus, Faun. Suec. p. 269. n. 1035 (1761).

Var. Pontia chariclea, Stephens, Ill. Haust. i. p. 17, pl. 3. figs. 1, 2 (1827).

Germany; France; Syria.

B.M.

We have a female specimen labelled St. Domingo ; but this must, I think, be an error.

\section{Synchlö̈ Crucivora.}

Pieris crucivora, Boisduval, Sp. Gén. Lép. i. p. 522 (1836).

Hakodadi; Nagasaki; Shang-hae.

B.M.

28. SynchloË ERgANe.

Papilio ergane, Hübner, Eur. Schmett. i. figs. 904-7 (1827?).

Pontia narcaa, Freyer, Neuere Beiträge, i. pl. 43. fig. 3 (1828).

Pieris rapa, var., Boisduval, Sp. Gén. Lép. i. p. 520 (1836).

Var. Pontia mannii, Mayer, Stett. ent. Zeit. p. 151 (1851).

Turkey.

29. SynchloË KrUePERI.

Pieris krueperi, Staudinger, Wien. ent. Mon. iv. p. 19 (1860);

Mill. Icon. iv. p. 259, pl. 1. figs. 3-5 (1863).

Var. Pieris krueperi, var. vernalis, Staud. Hor. Soc. Ent. Ross. p. 34 (1870).

Greece.

Intermediate between P. rapa and brassica.

30. Synchloe:? aglaope.

Pieris aglaope, Motschulsky, Etudes Entom. ix. p. 28 (1860).

Japan.

31. Synchloé Rape.

Pieris rapa, Linnæus, Faun. Suec. p. 270. n. 1036 (1761).

Var. Pontia metra, Steph. Ill. Haust. i. p. 19 (1827).

Var. Pieris rapa, var. leucotera, Stef. Bull. Ent. Soc. Ital. i. p. 147 (1869).

Italy ; Germany ; Syria ; Japan.

Proc. Zool. Soc. -1872 , No. V. 
32. SynchloË Castoria.

Pieris castoria, Reakirt, Proc. Acad. Nat. Sci. Philad. p. 238. n. 2 (1866).

Pieris reseda, Boisduval, Lép. Cal. p. 39. n. 8 (1869).

California.

Allied to S. rapa.

33. SynchloË Canidia.

Papilio canidia, Sparrman, Amœn. Acad. vii. p. 504, note m. (1768).

Papilio gliciria, Cramer, Pap. Exot. ii. pl. 171. figs. E, F (1779).

Papilio liciria, Herbst, Natursyst. Schmett. v. p. 63. n. 2.

Pieris glaphyra, Godart, Enc. Méth. ix. p. 160. n. 140 (1819).

Var. ㅇ? Papilio acaste, Linnæus, Mus. Ulr. p. 250 (1764).

Punjaub ; Silhet; China; Hong-Kong; Amoy.

\section{SynchloË monuste.}

Papilio monuste, Linnæus, Mus. Lud. Ulr. p. 237 (1864).

Papilio phileta, Fabricius, Syst. Ent. p. 47 l. n. 119 (1775).

Papilio monusta (sic), Herbst, Natursyst. Schmett. pl. 88. fig. 6 .

Mylothris hippomonuste, Hübner, Verz. bek. Schm.p. 91 .n. 952 (1816).

Pieris orseis, Godart, Enc. Méth. ix. p. 141. n. 78 (1819).

Pontia feronia, Stephens, Ill. Haust. i. p. 149 (1828).

Var. Papilio albusta, Sepp, Surin. Vlind. iii. pl. 141 (1855).

Pieris mandela, Felder, Wien. ent. Mon. v. p. 80. n. 30 (1861).

Pernambuco ; Brazil.

B.M.

35. SynchloË suasa.

Pieris suasa, Boisduval, Sp. Gén. Lép. i. p. 549. n. 160 (1836).

Peru.

B.M.

Possibly a melanistic form of $S$. monuste.

36. Synchlö̈ Joppe.

Pieris joppe, Boisduval, Sp. Gén. Lép. i. p. 495. n. 87 (1836);

Lucas in Ramon de la Sagra's Hist. Cuba, vii. p. 491, pl. 15. figs. $2,2 a(1857)$.

Jamaica.

B.M.

Allied to S. monuste.

\section{Synchlö̈ Cleomes.}

Pieris cleomes, Boisduval \& Leconte, Lép. Am. Sept. p. 43, pl. 16 (1833).

?Pieris vallei, Boisduval, Sp. Gén. Lép. i. p. 494. n. 86 (1836);

Lucas in Ramon de la Sagra's Hist. Cuba, vii. p. 491, pl. 1. figs. 1, $1 a$ (1857).

Honduras; Nicaragua; Portorico.

B.M. 
38. SynChloË virginia.

Pieris virginia, Godart, Enc. Méth. ix. p. 141. n. 81 (1819).

Mylothris hemithea, Hübner, Zuträge ex. Schmett. figs. 693, 694 (1832).

Venezuela; West Indies.

B.M.

Genus 14. Tatochila, Butler.

Cist. Ent. iii. pp. $38 \& 51$ (1870).

\section{Tatochila autodice.}

Synchloë autodice, Hübner, Samml. ex. Schmett. ii. pl. 127 (1816-36).

Chili ; Bolivia.

B.M.

2. Tatochila theodice.

Pieris theodice, Boisduval, Voy. de l'Astr. Lép. p. 51. n. 11 (1832); Gay's Faun. Chil. vii. p. 12, pl. 1. figs. $1 a, b$ (1852).

Peru.

B.M.

3. Tatochila microdice.

Pieris microdice, Blanchard, Gay's Faun. Chil. p. 14 (1852).

Chili.

\section{Tatochila mercedis.}

Pontia mercedis, Eschscholtz, Kotzeb. Reise, iii. p. 215, pl. 9. figs. $22 a, b$ (1821).

Chili ; Peru.

B.M.

5. Tatochila demodice.

Pieris demodice, Blanchard, Gay's Faun. Chil. p. 13 (1852).

Sandy Point; Port Famine.

B.M.

6. Tatochila xanthodice.

Pieris xanthodice, Lucas, Rev. Zool. p. 337 (1852).

Var. ?Pieris polydice, Blanchard, Gay's Faun. Chil.p. 12 (1852). Quito.

Genus 15. Herpenia, Butler.

B.M.

Cist. Ent. iii. pp. $38 \& 52$ (1870).

1. Herpenia eriphia.

Pieris eriphia, Godart, Enc. Méth. ix. p. 157. n. 134 (1819);

Lucas, Lép. Exot. pl. 28. fig. 2 (1835).

Pontia tritogenia, Klug, Symb. Phys. pl. 7. figs. 18, 19 (1829).

Congo.

B.M.

P.S. Several species of Pierides from Costa Rica have been described in the fourth part of 'Cistula Entomologica' for January 1872 , subsequently to the reading of this paper. 


\section{Notes on the Raptorial Birds of India. By A. Anderson, F.Z.S.-Part II.*}

[Received January 4, 1872.]

\section{Aquila fulvescens, Gray. (The Indian Tawny Eagle.)}

Met with everywhere. In some places almost abundant, as well as a nuisance, from the clanging noise it makes when depriving other birds of their food. The habits of this Eagle have been well described by Jerdon; and the bird is probably too well known to require special notice. I examined several nests during the season, and invariably found only two eggs. They vary considerably in size, shape, and coloratiou; but on the whole they are poorly marked. January and February is the most general time for this Eagle to lay ; but I came across some nests early in November. These birds had evidently built too soon, and used to sit mopingly close to their nest or on a neighbouring tree, as if watching their homestead, patiently waiting their appointed time.

The Wokab is partial to certain trees for the site of its nest; but I have found its predilection in this respect to be regulated by the abundance or scarcity of the trees in question. In the Cawnpore district they almost invariably build on solitary peepul trees (Ficus religiosa). In the Futtehgurh and Mynpoory districts, where the seesso (Dalbergia seesoo) grows to so gigantic a size, the preference is apparently given to them. Higher up the Doab, where the country assumes somewhat of a desert character, I found them building on thorny acacias. On one occasion I found a nest on a babool, which was certainly not more than fifteen feet high-a mere apology for a tree.

In November, 1867, I got a pair of abnormally small eggs, without the faintest indication of any colouring-matter (the contents of an unusually small nest, which was situated at the very top of a perpendicular branch of a mango), shooting one of the parent birds. This tree was one of a straggling group, close to the Martinière College at Lucknow ; and, in proof of the boldness of this Eagle, I may mention that an enormous camp was formed under these very trees, awaiting the triumphal entry of the Viceroy into the capital of Oudh. I have since thought that this nest belonged rightfully either to Milvus govinda or to Haliastur indus, both of which species were very abundant there. When encamped at the pretty little station of Mynpoory in January last, a pair of Wokabs became excessively troublesome, carrying off every thing they could find, and robbing the more legitimate camp-scavengers, Kites and Crows, of every morsel they picked up. I was not long in finding their nest, an enormous structure, on the topmost branches of a seesoo, which was visible nearly a mile off, as at this season of the year the tree was devoid of every green leaf. The nest contained two halfgrown eaglets, which were most tenderly nurtured by their parents,

\footnotetext{
* For Part I., see P. Z. S. 1871, p. 675.
} 
judging from the frequency of their visits and the pugnacious way in which they attacked every bird that unconsciously approached within sight, no matter how far off. During one of my visits to the tree, I saw both the birds in hot pursuit of a Jugger Falcon that was flying away with a Pigeon. Another day I wounded a Poliornis teesa, which flew away dangling both legs. Simultaneously with my shot out flew one of these Wokabs, and pursued the wounded Buzzard, in the vain hope of becoming possessed of its prey! The Eagle very soon overtook the unfortunate bird, flying round it several times by way of inspection, and when satisfied that no booty was forthcoming, it returned to the nest after two or three rapid gyrations.

As mentioned by Jerdon, the Wokab subsists to a great extent by robbing other birds of prey of their earnings. The stomach of one I examined contained a large Field-Rat (Gerbillus indicus); the head and shoulders had been swallowed whole; and the tail alone measured eight inches long. Not long ago I shot one in the act of eating a Wild Pigeon (Columba intermedia), which must have been caught out of a flock of several thousands which were settling on a few solitary trees for the purpose of roosting.

I have said that probably no two birds approach each other more in some phases of plumage than do the Indian Tawny (A. fulvescens) and the European Tawny (A. navioides) Eagles. But a remarkable characteristic in these two birds is, that their adult livery is assumed in reverse ratio to each other. Aquila fulvescens gets paler as it advances in age ; and I have specimens of a uniform cream-colour, showing every signs of age, very like the second figure of Aquila navioides in 'The Ibis' for 1865, page 166 . The latter, per contra, is pale buff when immature, and gradually assumes a rich chocolatebrown when fully adult. There is no doubt, however, that $\boldsymbol{A}$. fulvescens might be mistaken at a distance, either in its dark brown or pale buff stage for $A$. ncvioides; and many a one $\mathrm{I}$ have shot in the hope of finding a specimen of the latter bird.

That the Wokab can be mistaken for something even better than the $A$. navioides is proved by the sequel; for, since the first portion of these "Notes" was written, I shot a dark-looking bird off a telegraph-post, on the 28th of September last, and found myself in possession of a beautifully spotted (immature) female specimen of that somewhat mystical bird Aquila hastata, the first example I had ever seen in the flesh.

\section{Aquila hastata, Less. (The Long-legged Eagle.)}

I did not anticipate the gratification of being able to include this rare and interesting bird in the present paper when I first undertook it, a gratification which has been very considerably increased by my being already in a position to furnish Messrs. Gurney and Tristram with specimens of my own shooting. As I believe this Eagle is not at present represented in any of our British museums, and as the author of 'Rough Notes' alludes to it as a "doubtful species" *, I

* Rough Notes, pt. i. p. 181. 
trust no time will now be lost in having Aquila hastata figured, so as to enable him to speak for himself!

I have been extremely fortunate in procuring a good series of this desideratissimum of all Indian Eagles, as my collection already contains five specimens-representing both sexes, as also several phases of plumage.

No one that has ever seen this handsome little Eagle in the flesh could ever dispute its distinctness from both Aquila navia and $\boldsymbol{A}$. fulvescens. Structurally it has its affinities with the former, as the nostril is round, though smaller and more circular, and it has the same soft, silky plumage. The fully adult bird might be mistaken for a pale, spotless $A$. navia ; but the feeble beak, slender tarsi, length of wings, which generally exceed the tail, to say nothing of the comparatively small size of the whole bird (if the sex has been determined), are more than sufficient to separate it at a glance. The immature bird can never be mistaken for A. navia, as the minute white or fulvous dots on the bend of the wing, which are confined to the wing-coverts, chiefly near the carpal joint, and the pale fawn colour of the under wing-coverts, together with the above characteristics, distinguish it at once. Still less can I understand how it could be confounded with $A$. fulvescens; for, leaving alone the question of plumage, it is structurally distinct. Had Mr. Hume examined the nostril of the disputed bird in Col. Tytler's museum, he would have noticed that, although it was "vastly like A. fulvescens," the nostril alone was sufficient to separate it from that bird.

In its habits Aquila hastata is equally distinct from either of the above species. It affects open, alluvial country, is never found (according to my present experience), like the Wokab, in dry, arid plains, nor in the vicinity of stagnant, marshy pools, like the Spotted Eagle. All my specimens have been procured (by me) in low, open country in the vicinity of rivers, which was more or less submerged during the rainy season, and was only then being ploughed for the first time since the cold weather had set in. I never once saw a single A. navia in the same country as where I met with $A$. hastata, although the Wokab was abundant.

My best collecting-ground for the Long-legged Eagle (decidedly a misnomer, as the measurements will prove) was between the Ganges and Ramgunga, in the Futtehgurh district. The country between these two rivers is low, and is subject to inundation more or less every year. This season, owing to the unusually heavy rains, the two rivers were one continuous sheet of water for two months, submerging a strip of country from six to eight miles in breadth. It was here that I got my first specimen, seated on a telegraphpost within shot of the road, on the 28th of September, before the waters had fairly subsided.

Since procuring the above specimens, I have explored the most likely ground in the Etawah and Mynpoory districts ; and although I have met with Aquila fulvescens everywhere, and Aquila naevia in all suitable ground, neither Mr. Brooks nor I have as yet succeeded in adding Aquila hastata to the avifauna of either of these districts. 
The natural conclusion we have arrived at is, that this part of the country is too dry. I did indulge the hope that, like its ally the Spotted Eagle, it too would be a marsh-loving bird; but I think it pretty safe to conclude that the two Eagles do not affect the same kind of ground. Time and further research, however, is necessary before this point can be authoritatively settled; and it is just possible, now that we know $A$. hastata, that the bird may yet occur as a rare straggler near the jheels as the weather gets warmer.

It is a matter of regret that we are still ignorant as to the food of this Eagle; for the craws of my specimens did not contain the slightest vestige of any thing, notwithstanding I made careful postmortems. In the stomach of the first bird I found an intestinal worm $3 \frac{1}{2}$ inches long, which, together with all the sterna, has been preserved. They were all shot about two hours after sunrise, by which time Raptores in this country have generally made their morning meal. It is, however, very probable that grubs and earthworms may constitute a good portion of their diet, and hence perhaps the reason they affect newly ploughed lands.

There are two more points connected with this bird which I should mention-namely, its heavy, slow, Kite-like flight, and its excessive tameness. Had this Eagle been in the least degree wary, I should never have succeeded in securing a single specimen, owing to the exposed trees they sit on, and the entire absence of any cover. I saw only five birds, and shot them all, at a distance of 20 to 25 yards; and I may add that no doubtful-looking one was passed over.

With regard to its general plumage, Aquila hastata, like Aquila navia, has two distinct stages, viz. the "spotted" and uniform pale brown. It is, indeed, a "Spotted Eagle," just as much as the latter, and, like it, probably takes several years before it assumes its fully mature dress. The length of the wings, which fully equal or exceed the tail, and the slight difference in the size of the sexes, are remarkable features in this bird. To make its history as complete as possible, I sent all my specimens to Mr. Brooks, C.E., Etawah, and I am indebted to him for the following descriptions of them. I was very near omitting to mention that, as far as I am aware, these specimens are the first as yet recorded from the North-Western Provinces, strictly so defined.

A (q). Futtehgurh district, extreme north, 28th September, 1871.-Cere and gape lemon-yellow; bill pale plumbeous blue at base, rest horny black ; irides hazel-brown; feet pale yellow.

General colour of plumage, above and below, as far as vent, hairbrown ; it is, however, in the moult, and the feathers are of different shades. Many of the head-feathers and neck-hackles have pale tips. The bend of the wing, as also the shoulder, has a spotted appearance, as most of the lesser wing-coverts are tipped with dull white or pale fulvous. Upper tail-coverts light brown, and almost white at junction with tail ; most of them have white tips. Tail plain hoary brown, darker along the shafts of the feathers; some of them show indistinct whitish, greyish, or hoary bars, which are 
almost square to the shaft, but are very slightly inclined upwards. The uppermost tail-feathers do not show any barring; and the lower surface is of a uniform hair-brown. The lower tail-coverts are paler, and a good deal mottled and tipped with brownish white. The under surface of the tail shows the bars very distinctly in all the feathers. The tarsi are whitish brown, streaked with darker brown. Most of the primaries and secondaries are barred in their inner webs; lining of wing pale brown, mottled with brownish white. It is probable that some of the longer primaries in this specimen may not be fully grown. There is a strong purple gloss on the upper plumage, especially on the tertials. The spots on the wings of this moulting bird are of a rich buff on the new feathers, and they are almost white in the old ones, sufficiently proving that the spots are not lost in one moult. The new feathers are also just as freely spotted as the old ones.

B $\left(\delta^{*}\right)$. Futtehgurh district, two miles south of the Ganges, 28th October 1871.-Cere, gape, and angle of eye lemon-yellow; feet the same; irides light yellow; bill, basal half light plumbeous blue, rest blackish.

This specimen differs from specimen $\mathrm{A}$ in having its moult further advanced; and the upper surface generally is of a darker hairbrown. The tips of yellow-brown on the head are more distinct; and the spots on the wings are much more profuse. At the bend of the wing and along the ridge to the shoulder they are quite confluent. Those on the new feathers are ruddy or buff-white, while those on the old ones have faded to quite white. These spots extend to all the coverts of the wing, so that it is, to a certain extent, quite a "Spotted Eagle ;" they are, however, confined to the wing-coverts. The upper tail-coverts are the same as in specimen A-namely, very pale brown, tipped with white. Tail also hoary brown, barred as in specimen $\mathrm{A}$. The bars, however, do not extend right across the feathers, but occupy the central part. They slightly incline upwards, and are greyish white. The ends of the secondaries in both birds are very pale brown ; lining of wing brown, mottled with pale yellowbrown. Tarsi pale yellowish brown, marked with darker brown, in the form of bars. Lower tail-coverts largely marked with brownish white, so as to present a much lighter appearance than the rest of the bird. The abruptly defined white borders to the ends of the upper tail-coverts are a peculiarity of this Eagle.

C ( 9 ). Raepore, Central India (in Mr. Brooks's collection).This is very similar to specimen A. It appears, however, to be older, and was shot in March. The well-defined white borders to upper tail-coverts are worn off, leaving the ends of a brownish-white appearance; they are rather ragged. This bird has the pale tips to the tibial and tarsal plumes very distinct. There are a few pale tips to some of the head-feathers; but they are very minute, about $\frac{1}{20}$ of an inch across.

D (o). Futtehgurh district, six miles north of the Ganges, 6th November, 1871.-Soft parts ut suprà. This bird differs from specimen $\mathrm{C}$ in being profusely spotted on the wings; every covert- 
feather has a whitish spot at the tip, and so have the last two tertials. The colour of the tertials is a very pale brown. The spots on the wing are most numerous from its bend to the junction of the body. One or two of the upper scapulars are pale-tipped. Nearly all the feathers of the head and neck-hackles have minute pale tips, the size of a pin's head. Most of the abdominal feathers have pale tips; and the tibial and tarsal plumes are all edged with this pale rufous brown. The lower part of the tarsus is a light sandy brown. The white borders to the upper tail-coverts are most conspicuous. There is very little indication of barring on the central tail-feathers; but on opening the tail the other feathers are seen to be very distinctly barred with hoary grey.

E $\left(\sigma^{*}\right)$. Futtehgurh district, four miles north of the Ganges, 8th November, 1871.-This is an older bird, and is devoid of spots, except at the bend of the ridge of the wing to its junction with the body. On the latter part, or ridge, the spots are so thick as to be confluent (I ought to mention that this is the case in all the specimens as yet noticed). This specimen has no pale tips to the head-feathers; the well-defined white border to upper tail-coverts has disappeared, and these feathers are now simply very pale brown, with dark shafts, shaded to dull white at the edges and tips. Tail hoary brown, showing no bars above; it is only on opening them that very obsolete bars are visible in the inner webs of the outer feathers. The under surface of this bird is of a very uniform hair-brown, paling slightly towards the vent, as also on the tibia and tarsi, the latter being quite sandy on its lower half. There are no pale tips to the abdominal feathers; and those on the tibial and tarsal plumes have nearly all disappeared. The lower tail-coverts are pale brown, mottled with white. This specimen is in the moult, and the whole bird shows a mixture of old and new feathers. Some of the tailfeathers are only half-grown.

F ( 9 ). Futtehgurh district, extreme south, on the banks of the Kalee Nuddee (river), 19th November, 1871.-This is a still more mature bird; the spots on the wings have all vanished. The upper tail-coverts are pale brown, with darker central line; there are no light edges or tips to abdominal feathers, nor are there any light edges to the tibial and tarsal plumes; the latter are plain brown to junction with foot. Lower tail-coverts light brown, with dark central stripes; towards the tips they are rather pale. Feathers on the top of the head indistinctly tipped with light brown. Tail, as seen from above, without bars; but obsolete barring is visible on opening it. This specimen is also in the moult; the whole bird, especially the coverts of the wings, is of different shades of brown. It is, however, generally a darker bird than any of the others, being almost as dark as some specimens of mature A. navia.

As is the case with Aquila navia, this Eagle also is subject to a strong purple gloss, and, like it, has the plumage soft and silky. The primaries and secondaries in the fully adult bird, No. 6, are not barred on the inner webs as in A. fulvescens, but are plain as in 
adult $A$. navia. The nostril is even more circular than in that bird (A. navia), being a very broad ellipse, slightly inclining forwards. The tibial plumes in Aquila hastata are scanty, and very different from the well-furnished legs of $A$. imperialis and $A$. fulvescens.

Subjoined are the measurements of the above six specimens :-

\begin{tabular}{|c|c|c|c|c|c|c|c|c|c|c|c|c|c|c|}
\hline  & $\begin{array}{l}1 \\
0 \\
0\end{array}$ & $\frac{\sqrt{9}}{80}$ & 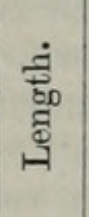 & $\stackrel{\nexists}{\exists}$ & స్ &  & 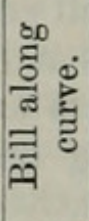 &  &  & 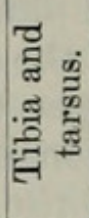 & 胥 & 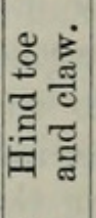 & 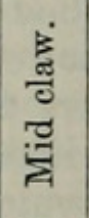 &  \\
\hline & 0 & lb. oz. & 94 & 18. & & $1.0)_{5}$ & 9.05 & 94 & $3.6^{\circ}$ & $8 \cdot 2)$ & $3 \cdot 05$ & D.? & $1 \cdot 1$ & 3 \\
\hline $\begin{array}{l}\text { A. } \\
\text { B. }\end{array}$ & $\begin{array}{l}+ \\
\text { o } \\
\end{array}$ & $\begin{array}{ll}0 & 0 \\
2 & 13\end{array}$ & 24.5 & 18.5 & $10 \cdot 0$ & 1.4 & $2 \cdot 2$ & $1 \cdot 0$ & 3.92 & 8.5 & $3 \cdot 1$ & $2 \cdot 23$ & $1 \cdot 27$ & $1 \cdot 25$ \\
\hline C. & † & $\ldots$ & $26 \cdot 0$ & $18 \cdot 5$ & $9 \cdot 25$ & $1 \cdot 2$ & 1.9 & 95 & $3 \cdot 75$ & $8 \cdot 5$ & $3 \cdot 05$ & $2 \cdot 2$ & $1 \cdot 1$ & $1 \cdot 3$ \\
\hline D. & o & $\begin{array}{lll}2 & 11\end{array}$ & $24 \cdot 0$ & $17 \cdot 8$ & $9 \cdot 25$ & $1 \cdot 3$ & $2 \cdot 05$ & 97 & 36 & $8 \cdot 5$ & $2 \cdot 9$ & $2 \cdot 1$ & $1 \cdot 17$ & $1 \cdot 15$ \\
\hline E. & के & $29 \frac{1}{2}$ & $25 \cdot 0$ & $18 \cdot 8$ & $10^{\circ} 0$ & 1.25 & 1.93 & 9 & $3 \cdot 6$ & $8 \cdot 7$ & $3 \cdot 0$ & $2 \cdot 4$ & $1 \cdot 17$ & $1 \cdot 4$ \\
\hline F. & 우 & $\begin{array}{ll}3 & 10\end{array}$ & $25 \cdot 5$ & $18 \cdot 75$ & $10 \cdot 25$ & $1 \cdot 22$ & $2 \cdot 03$ & $\cdot 97$ & $3 \cdot 8$ & $9 \cdot 1$ & $3 \cdot 3$ & $2 \cdot 1$ & $1 \cdot 3$ & 1.5 \\
\hline
\end{tabular}

Remarks.-A. Wings exceed tail by $\frac{1}{4}$ inch. B. Wings equal with tail. C. Mr. Brooks's bird. E. \& F. Wings exceed tail by $\frac{1}{4}$ inch.

31. Aquila pennata, Gmel. (The Booted Eagle.)

Decidedly rare in this part of the country. I procured two specimens ( $\sigma^{*}$ and $q$ ), the only ones I saw. The first is a fully mature bird with pure white abdomen ; the latter immature, having the underparts of a uniform dark brown.

My first introduction to this Eagle took place on the 18th March last. When driving along the Etawah branch canal, close to its junction with the Mynpoory road, I saw what at first appeared to me a Buzzard (Buteo ferox, a common bird in that neighbourhood), in pursuit of some insect on the ground, which was constantly escaping from its grasp. The bird was extremely wary, and I had great difficulty in approaching it within shot.

Dimensions (mature male). Length 21.5 inches ; wing 16 ; tarsi $2 \cdot 8$; foot, greatest length 4.5 , greatest breadth $3 \cdot 7$; mid toe to root of claw $1 \cdot 7$, its claw along curve $1 \cdot 2$; hind toe to root of claw 1 , its claw along curve $l .6$; bill, straight from base of cere to point $1 \cdot 5$, along curve $1 \cdot 6$, from gape $1 \cdot 6$, width at gape $1 \cdot 1$. Weight $1 \mathrm{lb} .13 \mathrm{oz}$.

Description. Cere and gape bright yellow; feet dirty yellow; irides pale brown ; bill blackish at the tips, basal half pale blue; the breast is light brown; abdomen pure white ; the white spots between the neck and wings were most conspicuous before the bird was skinned; and there is decidedly a rudimentary crest. The other characters are the black supercilium, streak between the lores and eyes, and dark stripe down the centre of the throat. All these points are equally present in the other bird, an immature female. I think it is safe to conclude that the white-bellied bird, as is the 
case with Eutolmaëtus bonellii and Pernis cristatu, is the adult garb, although this is at variance with Dr. Jerdon's opinion; and Mr. Hume inclines, I think, to the same belief.

\section{Eutolmaëtus bonelli, Temm. (Bonelli's Eagle.)}

This noble and magnificent Eagle is pretty generally distributed in suitable localities. I procured a good many specimens; and, with one exception, they were all in the mature dress. If the breadth of the striation on the under plumage is indicative of age, as is supposed to be the case by Mr. Brooks*, some of my birds must have been very old indeed, as the linear ovate marks on the tips of some of the feathers on the flanks and thighs were nearly a quarter of an inch broad.

My notes regarding this Eagle refer to their breding-season, January and February, during which period I invariably found them in pairs, or at no distance from each other. The only solitary example was the immature one above referred to ; and it must then have been one or two years old. From this it might be inferred that it does breed in the younger dress, as some Raptores are known to do. The under plumage of this one was of a uniform dark ruddy brown, the striation being very narrow, confined entirely to the shafts of the feathers. It is curious that, while in migratory Raptores (Aq. navia, navioides, imperialis, \&c.) the preponderance of young birds is so marked, the reverse should appear to be the case with this Eagle, a permanent resident. This, however, may be accounted for by the "Morunghee" in its plain brown stage being passed over at a distance for some other bird.

The straggling belt of dhak-jungle (Butea frondosa) which runs at broken intervals through the entire length of the Doab (N. W. P.), marking, as is supposed, the old bed of some river, is par excellence the favourite habitat of this noble bird. Here Aquila bonellii, seated on the summit of a lofty peepul or burgot seems to be monarch of all he surveys; and woe betide any luckless Peafowl, Hare (Lepus ruficaudatus), or Partridge (Ortygornis ponticeriana) that happens to come within tempting distance. In the course of a morning's march through this scrub-jungle, studded here and there with gigantic trees, and well stocked with game, I have seen two or three pairs. Each pair seems to hold sway over a circuit of two or three miles ; and they do not apparently intrude upon each other's hunting-grounds. Early in the morning they may be seen seated on the summit of some high tree which commands the neighbouring country; but if later in the day, they are on the wing, sailing over the jungle in search of food.

This dhak-jungle, besides furnishing these game-killing Raptores with such large quarry, encloses numerous shallow jheels, which are the favourite resort of aquatic birds early in the season, and it has consequently an additional charm for this rapacious Eagle, as well as for other birds of prey.

According to Mr. Hume's experience $\uparrow$, this Eagle would appear

$$
\text { * Ibis, } 1869 \text {, p. } 44 .
$$

$\dagger$ Rough Notes, pt. i. p. 189. 
to nidificate by preference on ledges of cliffs and old ruins. Doubtless it is a rock-building species; but it is one of those Raptores that adapts itself to circumstances, by building on trees near places that abound with its most natural food. I have no doubt that, in the absence of the above sites, they build commonly throughout the country I have alluded to, perhaps exclusively, on huge-limbed peepul and burgot trees.

I was particularly unfortunate with regard to the eggs of this Eagle, as the country was new to me, and I was just a little too late. The only eyry that rewarded my exertions (4th March) was placed on just such a tree as I have described, and contained a pair of "squabs" about a week old, for all the world like pure white powderpuffs. Even at that early age, there was no mistaking the characteristic legs and claws of young $A$. bonellii. I was very much struck by the affection shown by the female bird for her offspring; for she flew in and out of the tree even after I had twice wounded her, before I was aware that her nest was there. None of my men could climb the tree, owing to the enormous girth of the trunk; nor was any nest visible from below, as the foliage was dense, and the lower branches were covered with a thorny creeper. With the help of an extemporized ladder, ropes, \&c., I managed to reach to where the trunk bifurcated into two huge boughs, and from there saw the position and shape of the eyry, which was placed on a horizontal bough at no great distance from the ground; but it was completely hid from view.

My next acquaintance with young $A$. bonellii (17th March) took place under circumstances worth recording; for it is something quite new in the history of this Eagle for it to usurp the nest of another bird. The one in question belonged to Haliaêtus leucoryphus; and as it was tenanted, I had the curiosity to have it examined. Before my climber had ascended a few feet, out flew an old Bonelli's Eagle, leaving a fine fat young one, the only inmate of the nest. It succumbed at last to a surfeit of Crows and Doves, the only food my Shikaree would give it, as he maintained that was the most strengthening flesh for all Hawks.

It has been suggested to me that the nest above alluded to was built by the parent birds; but I am as positive as it is possible for one to be in such matters that it was an old one belonging to $H$. leucoryphus, whose young, even supposing the nest to have been occupied that year, must have flown a month or two earlier. There is no mistaking the bowl-shaped nest of this Sea-Eagle; and I am most familiar with it. In its position, shape, size, and architecture it differed most materially from that built by Bonelli's Eagle, which I had seen only a short time before, and was exactly similar to the scores of nests belonging to the other species which I have examined from time to time. Besides, this nest was placed on the very top of a leafless peepul, in a most public part of the canal-banks, which was a regular thoroughfare. No Bonelli's Eagle would have built in such a situation.

I have not seen this species in company with other Eagles. It is 
essentially a clean feeder, and never consorts with its brotherhood over a dead carcass. It is, however, worth mentioning that the craw of one which I shot was very much distended, and to my surprise contained the leg and foot complete of a Short-eared Owl (Otus brachyotus)! How such a morsel could have been digested it is impossible to say. The old Scotch proverb, “Hawks dinna pick out Hawk's een," if taken in a literal sense, is certainly not applicable in this case.

Since writing the above, I have obtained another immature example, which is certainly a year older than the one already mentioned, as the dark ruddy-brown plumage beneath has given place to a pale fawn-colour: and the vent and thigh-coverts are assuming a whitish appearance. Probably this bird does not assume its full adult plumage before the fourth year.

38. CircaĚtus gallicus, Gmel. (The Short-toed Eagle.)

Generally distributed in suitable localities. Lays a single white egg, from January to March, and generally selects a solitary tree in an open plain for its nest. This Eagle is easily distinguished, either when soaring or sitting on a tree; it is rarely seen in well-wooded country.

39. Spilornis cheela, Daudin. (The Crested Serpent-Eagle.)

By no means an uncommon bird, and has exactly the opposite habits of the preceding one. It is very local, and only met with in well-wooded, watery places. In short, it affects the same sort of ground as the Spotted Eagle; and, like it, subsists almost entirely on Green Frogs. It is a very tame bird, and sits in a slouching manner on a low bough of a tree overlooking some stagnant pool. I have never seen it on the wing, unless made to fly. Does not breed in the plains.

\section{Haliä̌tus leucoryphus. (Pallas's Sea-Eagle.)}

These fine birds are common, frequenting rivers, canals, and jheels. Although fish forms their chief sustenance, there is no doubt that they feed largely on aquatic birds; and, judging from their numbers, and the quantity of feathers one meets with along the edges of jheels, they must be the most destructive Eagles in the country. The nest of one examined contained the bones of the common Hare (Lepus ruficaudatus). Nidification commences early in November, and lasts till February. Two is the usual number of eggs they lay, exactly similar to those of Haliaëtus albicilla; but I have three taken from a nest not long ago; this, however, is very unusual.

Next to Gyps bengalensis, this is the earliest breeder among our Indian Raptores. A case has come to my knowledge of this Eagle completing the outer fabric of its nest as early as the 10th of October, and then actually forsaking that locality for about three weeks, when both birds suddenly returned and finished their homestead.

They build invariably on trees, on the banks of rivers, or close to jheels, which are thronged during the cold season with innumerable waterfowl, and thereby secure ample food for themselves and their 
offspring. During midday they frequently soar to a tremendous height, uttering a harsh, clanging cry, which can be heard a mile off. I have frequently been guided to their nest from their noise.

42 bis. Haliä̈tus albicilla ( $H$. pelagicus et $H$. brooksii, Hume). (The White-tailed Eagle.)

I once saw an immature bird of this species ; and as it was in company with a young $H$. leucoryphus, there was no doubt as to its identity. It was dreadfully wild and unsettled, not allowing me to approach within any thing like shot.

Several immature White-tailed Eagles have been seen and procured in the country to which these notes refer; and the recent capture of a mature specimen in the Punjab* places the identity of this species beyond doubt.

\section{Buteo ferox, Gmelin. (The Long-legged Buzzard.)}

This large and handsome Buzzard occurs in great numbers throughout all the districts of the North-western Provinces, affecting alike both moist and dry localities; it avoids, however, well wooded country. It is only a cold-weather visitant, arriving in October, and leaving again for their breeding-haunts by the first week in April.

In the arid dry plains of the Etawah and Mynpoory districts, where these birds occur in surprising numbers, they feed almost exclusively on desert-rats ; but in swampy localities their food consists to a great extent of frogs, crabs, \&c.

I have frequently found these Buzzards flying about late in the evening, even after dusk; but have rarely got within shot of them at that hour. Their acute sight and noiseless flight makes one believe they are somewhat crepuscular in their habits. These Buzzards are subject to almost more variation in their plumage than any other bird; but their transitional stages are as yet desiderata.

48. Poliornis teesa, Franklin. (The White-eyed Buzzard.)

Abundant, and appears to have no choice as to habitat, as they are found alike everywhere. Feeds on rats, mice, and all manner of crustaceans. Is extremely noisy and easily tamed. Breeds in March and April, laying usually three eggs, of the Goshawk type.

50. Crrcus cyaneus, Linn. (The Hen-Harrier.)

Rather rare.

51. Circus swainsoni, A. Smith. (The Pale-chested Harrier.)

Common. I have several times seen this Harrier in the act of killing small birds.

\section{Circus eruginosus, Linn. (The Marsh-Harrier.)}

Abundant in marshes and neighbourhood of jheels. Obtained in every variety of plumage, including a uniform dark brown, nearly

$$
\text { * Ibis, } 1871 \text {, p. } 404 .
$$


black, with pale fulvous nuchal spot. This variety is extremely rare. Feeds frequently on wounded Ducks and Teal, but never attempts to carry off the quarry, and eats it on the spot.

\section{Haliastur indus, Bodd. (The Brahminy Kite.)}

Essentially a marsh-bird ; but, though extremely common in Lower Bengal, is far from being so in the North-western Provinces, and then only in wet cultivation. Builds on high trees, invariably in the vicinity of water, constructing a nest very much after the fashion of the Common Kite.

Nidification commences about February; their eggs resemble poorly marked specimens of Buteo vulgaris; and they rarely lay more than a pair.

\section{Milvus govinda, Sykes. (The Common Kite.)}

Universally present. They commence building early in cold weather; but December is the most general time for them to lay. My first eggs last year were got on the 18th of November, and they must then have been a week old.

These birds are perfectly fearless, and breed by preference in the most densely populated parts of villages and bazars. The countless varieties of their eggs defy all description.

\section{6 bis. Milvus major, Hume. (The Larger Kite.)}

This, I am informed, is the common Kite of Cashmere, and it is only a cold-weather visitant to the plains of India. They are by no means uncommon; but there are probably few birds so difficult to procure, owing to their excessive wariness. Its slow heavy flight, together with the white under the wings, suffice to distinguish it at a glance.

In most of the specimens I have examined, the white under the wings is most conspicuous ; the inner webs of the primaries, as far as the emarginations, are more or less pure white. Some birds have this part mottled; but the white predominates.

I have just now an undoubted male of this species before me in the flesh of the following dimensions:-Length $24 \frac{1}{4}$, wing $19 \frac{1}{4}$, tail $12 \frac{3}{4}$. This is an unmistakable Milvus major, indeed a monster Kite.

I have, however, specimens of a Kite with all the characters of Milvus major, but considerably smaller. It is also a cold-weather visitant, and is equally shy as the former. Mr. Brooks has examined these birds in my collection, and agrees with me in referring them to another species : they may be Milvus affinis, or perhaps more probably $M$. melanotis of Temminck.

I shot a very fine female of this lesser Kite in my camp last year, close to the railway station at Etawah; it was in company with several of the common species, and attracted my notice for several days, owing to its large size and the white under the wings.

Undoubtedly we have three species of Kites in India, two of them being migratory. 
57. Pernis cristata, Cuvier. (The Crested Honey-Buzzard.)

Common, and is easily recognized on the wing, at any stage of plumage, by its peculiar flight. Seems to be an object of special aversion to Parrots (Palcoornis torquatus), which invariably pursue this species with every appearance of hatred. I have frequently watched a flock of Parrots in the act of pouncing down on one of these birds, even from a considerable height. The Honey-Buzzard seeks refuge by flight, but is immediately overtaken and mobbed by its pursuers, till at last it manages to conceal itself in some densely foliaged tree. I have found this species building in March and April, but have never succeeded in procuring their eggs.

The food of this Buzzard consists of honeycombs and insects. The craw of one I examined contained more than half a pound of wax, honey, and bees; the culmen and scale-like feathers of the head were quite sticky from the freshly eaten honey.

I once, however, saw this bird in the act of pursuing something in long grass, half flying and half running, which I think must have been a Leveret. Its erectile crest, golden-coloured eyes, and rapid movements gave it a very fine appearance. I believe it is not above eating small birds; for one perched one day on a small enclosure wall within a few paces of my tent, casting wistful glances at my chickens.

The jemadar of my guard, a high-caste Hindoo (Thakoor), picked up a specimen of this bird that I had thrown away; and, on my expressing my surprise at his touching it, he informed me that it was a mistake to consider this bird as belonging to the Hawkkind, as its food was pure honey, and, further, that it was delicious eating! He called it "Mud-kare," which signifies literally honeycomb-eater.

59. Elanus melanopterus, Daud. (The Black-winged Kite.)

This handsome little bird, the connecting-link as it were between the Harriers and Kites, affects open, marshy country, and is generally found seated on a small tree.

It is by no means a common hird in these parts; and though it is a permanent resident, we know next to nothing about its nidification. I believe the bird breeds in the Oudh Terai, as in the month of April one year, when out tiger-shooting in that locality, I saw great numbers of them, and am almost certain I once saw one fly off its nest.

The immature bird, in its mottled stage, has the iris yellow ; but every mature specimen that I have seen has it blood-red.

60. Strix indica, Blyth. (The Indian Screech-Owl.)

Common.

65. Bulacca ocellata, Lesson. (The Mottled Wood-Owl.)

Common. This species frequently builds a nest, laying usually a pair of eggs, generally in February and March. 
68. Отus вваснуотus, Gmel. (The Short-eared Owl.)

Abundant in cold-weather months, arriving in October, and leaving again by the end of March. Confines itself almost entirely to grass lands; in a day's shooting dozens are put up.

It flies remarkably well during the day; and it is by no means uncommon to see them soar almost out of sight, especially if attacked by Crows and King Crows. Owl.)

69. Ascalapha bengalensis, Franklin. (The Rock Horned

Common.

70. Ascalapha coromanda. (The Dusky Horned Owl.)

Abundant. Lays generally two eggs in December and January. I have taken their eggs from the old nest of a Kite, as also from the bare fork of a tree.

I was once riding along the banks of the Cawnpore Branch Canal late in the evening, quite dusk, at low-water mark, when I was suddenly startled by the cry of a Heron in distress, which flew past me, under the level of the bank, close to the surface of the water, hotly pursued by one of these Owls. I immediately galloped after the birds, and by dint of shouting and screaming made the Owl give up the chase. Judging from the numbers of feathers of Herons and Egrets which one meets with along the canals, there is reason to conclude that these birds are commonly preyed upon by this powerful Owl.

\section{Ketupa ceylonensis. (The Brown Fish-Owl.)}

Pretty generally distributed, in suitable localities.

\section{Athene brama, Temm. (The Spotted Owlet.)}

Abundant. The roof of my present bungalow, as well as that of the Club-house at Futtehgurh, is infested by them; they are a dreadful nuisance at times; and there is no getting rid of them.

Breeds from January to March, laying usually four eggs, exactly similar to those of Scops aldrovandi. I have found this species breeding alike in holes of trees and eaves of houses.

They are excessively fond of flying about during the middle of the day, and sally forth long before dusk.

3. A List of the Cypraide found on the Coast* of New South Wales. By John Brazier, C.M.Z.S., M.R.S.N.S.W.

[Received January 2, 1872.]

During a tour of five months through the northern parts of New South Wales in 1870 , I travelled over 300 miles of coast-line, and

* The extent of coast-line of New South Wales commences at Point Danger, in latitude $28^{\circ} 8^{\prime} \mathrm{S}$., and terminates at Cape Howe, in latitude $37^{\circ} 30^{\prime} \mathrm{S}$.

Proc. Zool. Soc.-1872, No. VI. 
MR, J. BRAZIER ON NEW-SOUTH-WALES CYPREIDA. [Jan. 16,

found species that have never been recorded from this part of the world. It is of importance that those who from personal observation are in a position to do so should give to the scientific world the benefit of their researches, especially when they are able to correct errors, or to increase our knowledge of the geographical distribution of species.

\section{Genus CypraA, Linnæus.}

1. Cyprea carneola, Linn.

$H a b$. Bottle-and-Glass rocks, Port Jackson; Cape Banks, Botany Bay. This may be considered its furthest southern limit. It is very common to the north, at the mouth of the Macleay, Nambuccra, Bellinger, Redbank, and Clarence rivers.

2. Cyprea isabella, Linn.

Cypraa controversa, Gray.

Hab. Headland three miles north of the Nambuccra River, also Bellinger-River bar.

This is not the true C. isabella, Linn., but the variety named controversa by Gray. Rare here, but common in the Central Pacific Islands.

3. Cyprea fimbriata, Gmel.

Hab. Cape Solander, Botany Bay, Lake-Macquarie beach, and the mouth of the Clarence River.

4. Cyprea macula, A. Adams.

Hab. Botany Bay; Bottle-and-Glass rocks, Port Jackson; Broken Bay ; Newcastle ; Port Stephens ; Port Macquarie ; Macleay, Nambuccra, Bellinger, Redbank, and Clarence rivers; also Moreton Island in Moreton Bay.

5. Cyprea felina, Gray.

Hab. Twofold Bay, Botany Bay, Port Stephens, Port Macquarie, and at the mouths of all the rivers north of Port Macquarie.

6. Cyprea ursellus, Gmel.

$H a b$. Cape Banks, north head of Botany Bay.

7. Cyprea hirundo, Linn.

$H a b$. Redbank and Bellinger rivers.

8. Cyprea asellus, Linn.

Hab. Cape Banks, Botany Bay; Port Jackson; Port Stephens ; Port Macquarie; Nambuccra, Macleay, Bellinger, Redbank, and Clarence rivers.

Our specimens are larger and more deeply coloured than those from New Caledonia and Ceylon. I have not seen the species south of Botany Bay. 
9. Cyprea tabescens, Soland.

Hab. Broken Bay, Lake Macquarie, Port Stephens, and Port Macquarie; Macleay, Nambuccra, Bellinger, Redbank, and Clarence rivers.

10. Cypraa scurra, Chem.

Cypraa indica, Gmelin.

Hab. Broken Bay, north of Port Jackson.

\section{Subgenus Aricia, Gray.}

11. Cyprea arabica, Linn.

Hab. Cape Banks, north head of Botany Bay; Macleay, Nambuccra, Bellinger, Redbank, and Clarence rivers.

The specimens found at Botany were all young shells, and obtained, during a very low tide, under large stones.

12. Cyprea moneta, Linn.

Hab. Redbank River, twenty miles south of the Clarence River.

This species, so common in the tropics, is also common at the above locality; it is thrown on shore after gales, with the animal dead in the shells.

13. Cyprea annulus, Linn.

Hab. Cape Banks, Botany Bay; Bottle-and-Glass rocks, Port Jackson; Redbank River.

This species, in common with $C$. moneta, is found thrown up on the beach after gales, near the Clarence River.

14. Cyprea Caput-serpentis, Linn.

Hab. Cape Banks, Botany Bay; Shark Island, Port Jackson; Lake Macquarie; Broken Bay; Macleay, Nambuccra, Bellinger, Redbank, and Clarence rivers.

A specimen that I obtained at Botany Bay was jet-black. It is not found south of that place.

\section{Subgenus Luponia, Gray.}

15. Cyprea vitellus, Linn.

Hab. Cape Banks, Botany Bay; Schnapper Rock, Coogee Bay; Point Piper; Port Jackson; Broken Bay ; Port Stephens and Port Macquarie; Macleay, Nambuccra, Bellinger, Redbank, and Clarence rivers.

I have not found this species south of Botany Bay.

16. Cyprea lynx, Linn.

Cypraa vanelli, Linnæus.

Cypraa squalina, Gmelin.

Hab. Bellinger-River bar. 
17. Cyprea helvola, Linn.

Hab. Bellinger-River beaches.

18. Cyprea poraria, Linn.

Hab. Bellinger and Redbank rivers.

19. Cyprea Clandestina, Linn.

Cyprca moniliaris, Lamarck.

Hab. Cape Banks, Botany Bay; Watson's Bay, Port Jackson; Port Macquarie; Macleay, Nambuccra, Bellinger, Redbank, and Clarence rivers.

I obtained living examples of this species at Botany during a very low spring tide under stones, but have not seen it any further south.

20. Cyprea xanthodon, Gray.

Hab. Watson's Bay, Port Jackson (Mr. G. F. Angas) ; Broken Bay; Port Stephens; Port Macquarie; Macleay, Nambuccra, Bellinger, Redbank, Clarence, Richmond, and Tweed rivers.

This species I have very rarely found in good condition.

21. Cypraa subviridis, Reeve.

Hab. Port Stephens, Port Macquarie; Bellinger, Redbank, and Clarence rivers.

Mr. Sowerby, in his 'Thesaurus Conchyliorum,' erroneously states that this species inhabits the Cape-Verd Islands. New-Caledonian specimens are more pyriform and lighter in colour than those fonnd on the Australian coast.

22. Cyprea flaveola, Linn.

Hab. Botany-Bay Heads; Broken Bay; Lake Macquarie; Port Stephens; Port Macquarie; also Macleay, Nambuccra, Bellinger, Redbank, and Clarence rivers.

This species is not very common at any of the above localities.

23. Cyprea spurca, Linn.

Hab. Cape Solander, south head of Botany Bay (Mr. Hargraves); Newcastle beach, near Redhead, north of Port Jackson.

This species may be distinguished from C. flaveola and C. gangranosa by the back being always clouded and ocellated with yellow.

24. Cyprea lutea, Gronov.

Cypraa humphreysii, Gray.

Cypraa commixta, Wood. rivers.

Hab. Lake Macquarie; Newcastle beach; Redbank and Clarence

The variety humphreysii has the back of the shell olive-brown, with two narrow milky-blue zones, and comes from Nichol Bay, North-west Australia. 
25. Cypratea piperata, Soland.

$H a b$. Twofold Bay and Shoalhaven.

This species is also found at Port Lincoln, St. Vincent's Gulf, Port-Adelaide Creek, South Australia, and at Cape Riche, King George's Sound.

26. Cypraa bicolor, Gask.

Hab. Lake Macquarie and Bellinger River.

This species may be recognized by its pyriform shape and by the teeth being much finer than in C. piperata or C. comptoni. Some four years since some hundreds of $\boldsymbol{C}$. bicolor were washed on shore upon the outer beaches of Lake Macquarie, after an easterly gale of wind.

27. Cypraa comptoni, Gray.

Hab. Twofold Bay.

It is also found at Port Lincoln, South Australia ; Cape Riche, King George's Sound, and on the north coast of Tasmania.

28. Cyprea erosa, Linn.

Hab. Bottle-and-Glass rocks, Port Jackson; Broken Bay ; Port Stephens; Port Macquarie; Macleay, Nambuccra, Bellinger, Redbank, and Clarence rivers.

This species is found thrown up on all the outer beaches after the winter gales, and obtained alive under stones in the harbours.

29. Cyprea caurica, Linn.

Hab. Cape Banks, Botany Bay; Broken Bay.

This species, like the preceding, extends from Broken Bay northwards along New South Wales to Queensland.

30. Cyprea cruenta, Gmel.

Cyprea variolaria, Lamarck.

Hab. Bellinger and Redbank rivers; also a few dead specimens have been found at Lake Macquarie.

31. Cyprafa miliaris, Gmel.

Hab. Macleay River, under Grassy Head.

32. Cyprafer errones, Linn.

Cyprea olivacea, Lamarck.

Hab. Cape Banks; Botany Bay; Middle Harbour, Port Jackson; Broken Bay; Port Stephens; Port Macquarie, and all the other northern beaches of New South Wales.

Subgenus Cypraevula, Gray.

33. Cypreovula umbilicata, Sow.

Hab. Off Wollongong(?), deep water. 
"Several living specimens of this rare shell were dredged in deep water, at a distance of two miles off the coast, a little to the southwards of Wollongong, by Commodore Loring, C.B., when commanding H.M.S. 'Iris.' They are somewhat smaller and paler in colour than the ordinary Tasmanian examples.'-Mr. G. F. Angas, Proc. Zool. Soc. 1867.

\section{Genus Trivia, Gray.}

34. Trivia australis, Lam.

$H a b$. Shark and Clark Islands, Watson's Bay; Bottle-and-Glass rocks, Port Jackson; Lake Macquarie ; Port Stephens ; Port Macquarie; Macleay, Nambuccra, Bellinger, Redbank, and Clarence rivers. Tasmanian specimens are very large.

About four years ago there were some thousands of this species washed on shore at the outer beaches, Lake Macquarie, after an easterly gale.

\section{Trivia candidula, Gask.}

Hab. Bottle-and-Glass rocks, Port Jackson.

I obtained two living examples of this rare Trivia under a large stone during an unusually low spring tide.

36. Trivia globosa, Gray.

Hab. Cook's Landing Place, Botany Bay; and Little Bay, between Port-Jackson Heads and Botany.

37. Trivia insecta, Mighels.

Cypraa hordacea, Kien.

Hab. Little Bay, also Cabbage-Tree Bay, outside the north head of Port Jackson.

\section{Subgenus Pustularia, Swainson.}

38. Trivia limacina, Lam.

Cypraa interstincta, Wood.

Hab. Nambuccra and Bellinger rivers.

This species appears to be confused by some authors with the $C$. staphylae of Linnæus; but they are undoubtedly two distinct species, the teeth on the base of $C$. limacina being always large, and not extending across the base as in C. staphylaa.

39. Trivia staphylea, Linn.

$H a b$. Broken Bay and Newcastle.

This species is common on the New-South-Wales coast. 
4. On a fourth Collection of Birds from the Pelew and Mackenzie Islands. By Dr. G. Hartuaub, F.M.Z.S., and Dr. O. Finsch, C.M.Z.S.

[Received December 6, 1871.]

It was in the year 1867 that we had the first opportunity of reporting to this Society* on a small collection of birds from the Pelew and Mackenzie Islands, which, from their geographical position, may be considered of especial interest. These groups of islands are close to each other, connected by several small islets, and form the most western corner of the extensive archipelago of the Carolines. Since that date we have received two other collections from the Pelews t, forwarded to us through the Museum Godeffroyanum of Hamburg and the Museum of Altona (Dr. Semper), which have extended our knowledge of the avifauna of this group to a high degree. It is with great satisfaction that we now record the receipt of a new and still more complete collection from the localities mientioned above, sent to us for scientific determination by Mr. Johann Cesar Godeffroy, to whom science is greatly indebted for the exploration of some almost unknown groups of islands in the Pacific.

This collection was formed by Mr. Kubary (a young traveller in the service of Mr. Godeffroy, whom we had the pleasure of mentioning in terms of commendation in our last article on birds of Savai, P. Z. S. 1871, p. 22) on the island of Uap, of the Mackenzie group, and by Capts. Heinsoln and Peters, masters of vessels belonging to Mr. Godeffroy, partly at the same island, but chiefly on the Pelews. Thanks to the zeal and diligence of these gentlemen, we are now in the pleasing situation of being able to give a far more complete account of the ornithology of these interesting islands. Not only do we become better acquainted with some little-known species, but the total number of species has been increased. Amongst these additions no less than nine we have the pleasure of introducing as new ; and what is more important, some of them appertain to genera not yet known from this locality-such as Noctua, Caprimulgus, Campephaga, and Phlegoenas.

Our last list of Pelew birds embraced forty-one species, now we have to add eleven, making the total number fifty-two. Of the Mackenzie group or Ulathi, only the island of Uap has been explored. Here we were previously acquainted with six species only (Myzomela rubratra, Monarches godeffroyi, Calornis kittlitzi, Ardea sacra, Numenius phaopus, and Tachypetes minor); now we are able to enumerate twenty. The total number of known birds of this western portion of the Carolines is sixty-four; but of this number

* Dr. Hartlaub, "On a Collection of Birds from some less-known Localities in the Western Pacific," P. Z. S. 1867, pp. 828-832.

+ Drs. Hartlaub and Finsch, "On a Collection of Birds from the Pelew Islands," $i b .1868$, pp. 4-9, and "Additional Notes on the Ornithology of the Pelew Islands," $i b$. pp. 116-118. 
only two occur on both groups, and are widely distributed in the archipelago, namely Myzomela rubratra and Calornis kittlitzii. The rule in the geographical distribution of birds, that islands close to each other are generally inhabited by certain allied species of the same genus, which represent each other, is manifested very clearly with respect to the birds of the Pelew and Uap. The two islands have each two species of Zosterops, one Rhipidura, one Campephaga, and one Phlegonas. The avifauna of Pelew, hetter known than that of Uap, is richer in peculiar species, possessing twelve, whereas Uap has only six, which species are marked in our subjoined list with an asterisk. Of the other forty-six species known in these groups, twenty-seven are widely distributed over the Indo-Malayan region and the Pacific; but there is a strong tendency towards the birds of the former region, about sixteen being Indo-Malayan, whereas only seven are peculiar Pacific forms. Sixteen species may be regarded as stragglers, some of which are of very rare occurrence, such as Falco peregrinus and Nycticorax griseus, which visit these islands during their migrations. Nine of the species are European. Two species occur also in Australia.

In comparing the ornithology of these groups of islands with those of other Central-Polynesian groups, and taking into consideration their far less extent, we find that, nevertheless, they are considerably richer. Thus, the Vitis possess sixty species, amongst which eighteen are peculiar; whereas the Navigator group has only fifty, of which sixteen are peculiar; and the Friendly Islands, out of thirty-seven species, only six peculiar. This interesting fact, no doubt, must be considered a result of the far stronger influence of Indo-Malayan species which predominates in the western Carolines.

As a singular fact in relation to the general view of the ornithology of the Western Carolines, we may notice the absence of Fringilline birds and of Parrots, which, in respect of the latter, is the more remarkable, as we know of the occurrence of a very interesting species (Domicella rubiginosa) on the small island of Puinipet, of the Seniavin group, which forms the outermost eastern corner of the Carolines archipelago.

Mr. Kubary has given us some meagre notices about the island of Uap and its ornithic life. According to these the interior of Uap consists of a hill-like plateau, which is destitute of trees, and only covered with grass. This hilly interior is surrounded by a narrow strip of wooded land, of a garden-like appearance, not broader than from one to two English miles, which possesses a richer vegetation of areca-palms, banyans, bamboos, and, nearer to the shore, of cocoatrees. In general the vegetation is much poerer than in Upolu or other Central-Polynesian islands. On the plateau birds are very rare. Mr. Kubary notices only the Kuling (Strepsilas) and Numenius phroopus, and observed once a small bird, which he believes to have been a Collocalia, but which he was not fortunate enough to secure. The greatest amount of bird life is found in the wood-region. Here Myzomela, Zosterops, Campephaga, Phlegøenas, Ortygometra, and a species of half-wild Gallus occurs ; the Myzomela, 
Zosteropides, and Monarcha are not unfrequently seen near the houses of the natives. On the shores, Ardea sacra and lepida, Actitis incanus, and sea-birds (Sterna melanauchen, Gygis alba) are numerous.

We commence with a list of all the known species of western Carolinian birds :-

\begin{tabular}{|c|c|c|}
\hline & $\begin{array}{l}\text { Pelew } \\
\text { group. }\end{array}$ & $\begin{array}{c}\text { Mackenzie } \\
\text { group. }\end{array}$ \\
\hline 1. Falco peregrinus . & $\cdots$ & * \\
\hline *2. Noctua podargina & * & \\
\hline *3. Caprimulgus phalæna. & * & \\
\hline 4. Collocalia vanicorensis & * & \\
\hline 5. Haleyon chloris ..... & * & \\
\hline 6. — albicilla $\ldots . .$. & * & \\
\hline 7. — reichenbachi & * & \\
\hline 8. - sanctus............. & * & \\
\hline 9. Myzomela rubratra .. & * & * \\
\hline *10. Psamathia annæ ... & * & \\
\hline *11. Zosterops semperi & * & \\
\hline *12. — hypolais ...... & $\ldots$ & * \\
\hline *13. oleaginea ...... & $\ldots$ & * \\
\hline *14. finschi ...... & * & \\
\hline 15. Turdus obseurus & * & \\
\hline *16. Rhipidura lepida... & * & \\
\hline *17. $\quad$ versicolor........ & $\cdots$ & * \\
\hline *18. Myiagra erythrops. & * & \\
\hline *19. Monarches godeffroyi & $\ldots$ & * \\
\hline *20. Campephaga nesiotis & $\cdots$ & * \\
\hline *21. monacha ......... & * & \\
\hline 22. Artamus leucorhynchus & * & \\
\hline *23. Rectes tenebrosus ...... & * & \\
\hline 24. Calornis kittlitzi ......... & * & * \\
\hline 25. Cuculus canorus .......... & * & \\
\hline 26. — striatus ................ & * & \\
\hline *27. Ptilinopus pelewensis. & * & \\
\hline 28. Carpophaga oceanica .. & * & \\
\hline *29. Phlegœnas canifrons $\ldots . .$. & * & \\
\hline *30. yapensis ............ & $\ldots$ & * \\
\hline *31. Megapodius senex & * & \\
\hline 32. Gallus bankiva...... & * & \\
\hline 33. Charadrius fulvus & * & * \\
\hline 34. - geoffroyi .......... & * & \\
\hline 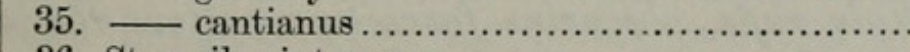 & * & \\
\hline 36. Strepsilas interpres..$\ldots \ldots \ldots \ldots \ldots \ldots \ldots \ldots \ldots$ & * & * \\
\hline 37. Ardea sacra .............. & * & * \\
\hline 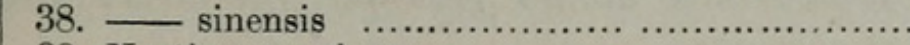 & $\ldots$ & * \\
\hline 39. Nycticorax griseus ............................. & $\ldots$ & * \\
\hline 40. - manillensis $\ldots \ldots \ldots \ldots \ldots \ldots \ldots \ldots \ldots$ & * & \\
\hline 41. & * & \\
\hline 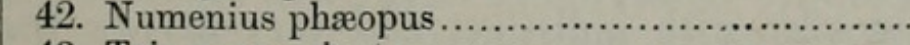 & * & * \\
\hline 43. Tringa acuminata $\ldots \ldots \ldots \ldots \ldots \ldots \ldots \ldots \ldots \ldots \ldots \ldots \ldots \ldots$ & * & \\
\hline  & * & \\
\hline 45. Actitis incanus $\ldots \ldots \ldots \ldots \ldots \ldots \ldots \ldots \ldots \ldots \ldots \ldots \ldots \ldots \ldots \ldots \ldots$ & $\cdots$ & * \\
\hline 46. $\subsetneq$ hypoleucus ....................... & * & \\
\hline (n) & * & \\
\hline 48. Rallus pectoralis ... & * & \\
\hline
\end{tabular}




\begin{tabular}{|c|c|c|}
\hline & $\begin{array}{l}\text { Pelew } \\
\text { group. }\end{array}$ & $\begin{array}{l}\text { Mackenzie } \\
\text { group. }\end{array}$ \\
\hline 49. Ortvgometra quadristrigata & * & * \\
\hline 50. Porphyrio melanonotus ..... & * & \\
\hline 51. Anas superciliosa ................ & * & \\
\hline 52. Fuligula cristata .................. & * & \\
\hline 53. Puffinus dichrous..................... & * & \\
\hline 54. Sterna longipennis ............... & * & \\
\hline 55. — melanauchen ... & * & * \\
\hline 56. — lunata ................ & * & \\
\hline 57. Anous stolidus & * & \\
\hline 58. $\square$ tenuirostris ........................ & * & \\
\hline 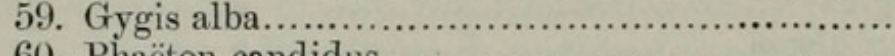 & * & * \\
\hline 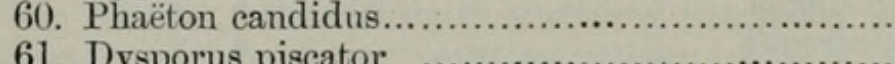 & * & \\
\hline 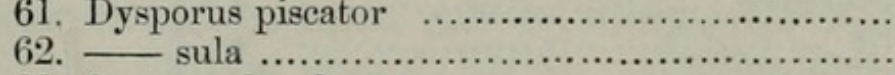 & * & \\
\hline 63. Carbo melanoleucus...................... & * & \\
\hline 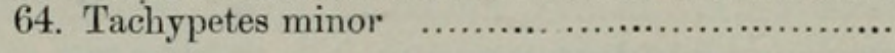 & $\cdots$ & * \\
\hline & 53 & 20 \\
\hline
\end{tabular}

\section{Falco peregrinus, L.}

A young male from Uap, captured during the north-east monsoon in November 1870 (Kubary). Not yet recorded from this locality.

Long. al. caud. rostr. tars. dig. med. ung.

$$
\begin{array}{ccccccc}
-6^{\prime \prime} & 6^{\prime \prime} & 4^{\prime \prime \prime} & 88^{\prime \prime \prime \prime} & 22^{\prime \prime \prime} & 22^{\prime \prime \prime} & 8^{\prime \prime \prime} \\
13 \frac{2}{2} & 6 & 10 & 10 & 23 & 25 & 9
\end{array} \text { (De Castries Bay.) }
$$

In a former collection, forwarded by Mr. Godeffroy, we received a similarly coloured specimen from De Castries Bay, east coast of Amoorland (Capt. Meyer).

\section{Noctua podargina, n. sp.}

Dilute ferrugineo-rufa, vix conspicue transversim variegata maculisque minutis albidis hinc inde notata; capite pallidius fulvescenite, indistincte fasciolato; uropygio et supracaudalibus crebrius albo maculatis; maculis nonnullis majoribus albis in alarum tectricibus; alis pallide rufis; remigibus irregulariter et rarius fasciatis, pogonio interno obsolete fuscescentibus; rectricibus pallide rufis, obscurius fasciatis; pectore et abdomine in fundo latius rufescenti-fulvo irregulariter fasciolato et maculato; subalaribus albido fuscoque fasciatis; rostro pallido; pedibus obscuris.

Long. tot. circa $11^{\prime \prime}$, rostr. a fr. $8^{\prime \prime \prime}$, al. $6^{\prime \prime} 2^{\prime \prime \prime}$, caud. $3^{\prime \prime}$, tars. $1^{\prime \prime}$.

One very indifferent specimen. The whole bird of an obsolete pale rufous colour, mottled with indistinct and very narrow transverse blackish and fulvous bands; inferior part of back, upper tailcoverts, wing-coverts, and scapularies irregularly marked with white sublanceolate or roundish greater or smailer spots; these spots are surrounded by a blackish margin; wing-feathers with a few indistinct and distant blackish bands, the inner vanes towards the base more 
blackish; under wing-coverts barred whitish and fuscous; feathers of the face, throat, and sides of head paler, distinctly and very narrowly banded; there appears to be a pale superciliary stripe; breast and abdomen pale rufescent, with blackish vermiculations and larger whitish darkly margined spots, or short, broad, transverse bands; beak pale yellowish; feet dark; the upper half of the tarsus is feathered.

The collection contains only a single specimen, collected by Capt. Heinsohn at the Pelews. All our efforts to refer this little Owl to any known species have been without success; we must therefore consider it to be new. Its nearest ally seems to be N. ochracea, Schleg., from Celebes. The specimen is apparently in full dress; but whether the rufous plumage may be only a phase, as in some other Owls, we are unable to decide. The light-coloured beak and the half-naked tarsi and toes, without any hairs, are especially to be noticed.

\section{Caprimulgus phalena, n. sp.}

Supra in fundo rufescente nigro transversim lineolatus striisque latioribus longitudinaliter notatus; uropygio et supracaudalibus distinctius fasciatis; remigibus majoribus nigris, medio dilute rufo bimaculatis; tectricibus et scapularibus pulchre et largius fulvo, rufo nigroque variegatis; subalaribus rufo nigroque fasciatis; macula jugulari alba; abdomine in fundo dilute rufescenti-fulvo fasciolis strictioribus nigris notato; subcaudalibus obsolete fulvo-albidis, rarius nigro fasciatis; rectricibus nigris, fasciis vermiculatis rufis maculatim notatis; rostro et pedibus nigris.

Long. tot. circa $10^{\prime \prime} 3^{\prime \prime \prime}$, rostr. a fr. $5^{\prime \prime \prime}$, al. $6^{\prime \prime} 3^{\prime \prime \prime}$, caud. $4^{\prime \prime} 4^{\prime \prime \prime}$, tarsi $6^{\prime \prime \prime}$.

The whole head above reddish brown, minutely mottled with dark brown, and each feather with a broad black shaft-stripe, forming three irregular longitudinal stripes; feathers of the hind neck and mantle dark rufous brown, with dusky patches along the shafts, and mottled indistinctly with dusky; feathers of the sides of neck with a large rufescent middle spot, forming a distinct patch, which is in connexion with the bright rufescent ear-region; larger shoulderfeathers rufous brown, towards the basal half light brownish grey, mottled minutely with dark brown, and with a large apical spot of black on the outer vane, which shows some small rufescent spots; this black is bordered on the upper shoulder-coverts externally broadly with light fulvous yellow, forming along the shoulders a black median stripe, edged externally by an indistinct fulvous-yellow line; rump and upper tail-coverts rufous brown, mottled with dusky, and barred with narrow black lines, more distant on the upper tailcoverts; primaries black, on the basal half of the outer web with three fulvous spots, of which the third is larger and principally visible; the other primaries have six rufous smaller marginal spots, which on the secondaries form irregular cross bands, the first four remiges with a fulvous median spot on the inner web; the remaining 
primaries with six rufescent spots internally, which on the secondaries form irregular narrow cross bands ; the last of the secondaries greyish fulvous, mottled with pale brown, and across the shaft with six black lines; the first quill has on the basal half four obsolete rufescent marginal spots; the shafts are black, and dark brown beneath; tectrices of the primaries black ; tectrices of the secondaries black, with rufescent marginal and apical spots, mottled minutely with dusky; the remaining upper wing-coverts are marked in the same manner, but the rufous spots are smaller, so that the smallest upper wing-coverts near the cubitus are black with narrow rufous edgings; the greater median wing-coverts show a large fulvescent apical patch on the outer web, forming a crossband-like conspicuous mark: under wing-coverts blackish brown, barred narrowly with rufous; feathers, the region beneath the eye, along the angle of mouth, chin and sides of throat rufous, with black apical edgings; throat apparently divided into two, with patches, by a narrow rufous black-barred median line ; crop and breast of a rufous-brown groundcolour, each feather towards the tip lighter, changing into greyish fulvescent, finely mottled with dark brown, forming speckled cross lines, and a narrow black shaft-stripe; remaining underparts pale fulvescent, with obsolete narrow dark cross lines, broader and more distinct on the under tail-coverts; three outer tail-feathers black, with nine rufous eross bands, which on the outmost are narrower (here ten), on the second and third broader, and mottled with dusky; on the inner web these rufous cross bands are only indistinctly indicated; the fourth tail-feather shows the nine rufous cross bands broader, distinct also on the inner web, and mottled more with dusky; the two middle tail-feathers have a rufous-brown groundcolour, internally changing into grey-brown, speckled with dusky, and nine irregular black bars. Bill black, bristles (eight) strongly developed, black; feet and nails hornish brown; the tarsus feathered at the basal half, rufescent ; shafts of the tail-feathers black.

$$
\begin{array}{ccccccc}
\text { Long. al. } & \text { caud. } & \text { rostr. } & \text { rict. } & \text { tars. } & \text { dig. med. } & \text { ung. } \\
6^{\prime \prime} 3^{\prime \prime \prime} & 4^{\prime \prime} 4^{\prime \prime \prime} & 5^{\prime \prime \prime} & 12 \frac{1}{2}^{\prime \prime \prime} & 6 \frac{1^{\prime \prime \prime}}{2} & 7 \frac{\frac{1}{}^{\prime \prime \prime}}{2} & 3^{\prime \prime \prime}
\end{array}
$$

Of this species a single specimen was collected by Capt. Heinsohn in the Pelews-a locality whence no Caprimulgine bird has been yet recorded. The specimen is evidently a female; and the male, no doubt, will present some considerable differences ; perhaps we may expect it will have the primaries as well as the outer tail-feathers spotted with white. Although female Caprimulgi are much more difficult to determine, and it might be considered somewhat hazardous to establish a species on an isolated specimen, nevertheless we are obliged to do so, having endeavoured to unite this specimen with any of the known species without success.

C. phalana seems to come nearest to $C$. jotaka, Temm., from Japan, and C. stictomus, Swinh. (Ibis, 1863, p. 250), from Taiwan, but may be distinguished, besides strongly contrasting differences in coloration, by its smaller size; the latter besides has naked tarsi.

Mr. G. R. Gray enumerates (Hand-1. of B. i. p. 57) two Night- 
jars from the Philippines, C. griseatus and C. manillensis, but both are still undescribed.

\section{Collocalia, sp.?}

Mr. Kubary observed once in the island of Uap a small bird, which he believes to have been a species of Collocalia.

5. Halcyon chloris (Bodd.); F. \& H. Vög. Ost-Afr. p. 165.

Halcyon albicilla, part., Hartl. P. Z. S. 1867, p. 828 (green-headed spec.); H. \& F. $i b .1868$, p. 4 ; Sharpe, Kingfishers, t. 73 (hind fig.).

In our first publication on the Birds of the Pelew Islands we noticed, under the head of $H$. albicilla, besides specimens in the normal white-headed plumage, some which showed more or less greenishblue feathers on the crown, and one with the whole upper head uniform greenish blue like the back, supposing this latter to be the young bird. Unfortunately we had overlooked that the young of the true H. albicilla had been already made known by Prof. Schlegel (Vogels van Nederl. Indië, Ijsvogels, p. 32, pl. 11. f. 4), who received a very young specimen, shot by the late Dr. Bernstein the 12 th September, 1861, in the island of Morotai. This specimen, represented in the plate cited above, resembles very much the old bird, having already the head white, but with some greenish-blue feathers on the top, like those we received from the Pelews. In a second collection from this locality we got three green-headed specimens; and the last collection (Capts. Heinsohn and Peters) contains ten specimens, all with greenish-blue heads.

From this excellent and instructive series we learn that we were quite mistaken in supposing the green-headed birds to be the young of $H$. albicilla, and that they belong to a distinct species, which we are not able to distinguish from the widely distributed $H$. chloris. We have before us five old specimens from India and Java which agree in every respect with them. The blue of head and back in some specimens changes more or less into bluish green; but there are all sorts of intermediate forms between the birds with blue and those with green back; the black band through the eyes and round the nape, aswell as the white occipital spot, are also more or less developed, the feathers of the former are more or less tipped with greenish blue. Three other specimens show certain signs of the young bird, in having the feathers of the sides of neck and breast, as well as those of the white neck-collar, with narrow dark edgings, giving them an undulating appearance; in these the whole head above is darker and of a dull olive-green; in one the white mark behind the nostrils is tinged with pale buff. Two younger specimens with quills not fullgrown, and tail-feathers, and short hornish-white-tipped bills, so characteristic of young Kingfishers generally, resemble altogether the young of the true chloris. The black surrounding the nape forms a broader patch ; the line above the loral region, as well as the breast and sides of belly, are tinged faintly with buff, and bear narrow blackish margins.

We must remark that the Pelew specimens seem to be generally 
of a larger size; but after comparing them with the valuable measurements given by Prof. Schlegel, as reproduced hereafter, we see no necessity for separating them as a distinct species, though, perhaps, some naturalists, not having specimens enough at their disposal, might be willing to do so.

H. chloris breeds on the Pelew Islands; the collection contains a single egg, which is of a uniform white.

A closely allied species is our $H$. cassini (Orn. Centr. Polyn. p. 40 ; Cass. Un. St. Expl. Exp. pl. 16. f. 1), which the excellent monographer of the Kingfishers declares to be "certainly identical" with H. sacra. This he must allow us to doubt. We have examined extensive series of $H$. sacra from the Navigators' and the Tonga group, but have never seen any authentic specimen from the Viti group belonging to that species. All the specimens from the latter group were found to belong to $H$. cassini; so we are of opinion that this species will prove to be peculiar to the Vitis.

\begin{tabular}{|c|c|c|c|c|c|c|}
\hline Long al. & caud. & rostr. & $\begin{array}{l}\text { t. } \\
\text { tr. }\end{array}$ & Tars. & $\begin{array}{l}\text { Dig. } \\
\text { med. }\end{array}$ & \\
\hline $\begin{array}{rrr}4 & 11-4 & 6 \\
3 & 7-3 & 9 \\
3 & 9-4 & 0 \\
3 & 11-4 & 1 \\
4 & 0-4 & 2 \\
& 3 & 10 \\
3 & 11-4 & 0 \\
3 & 11-4 & 1 \\
& 4 & 2 \\
& 4 & 1 \\
4 & 0-4 & 3 \\
& 4 & 3 \\
& 3 & 10 \\
& 3 & 10 \\
& 3 & 5 \\
& 3 & 11\end{array}$ & 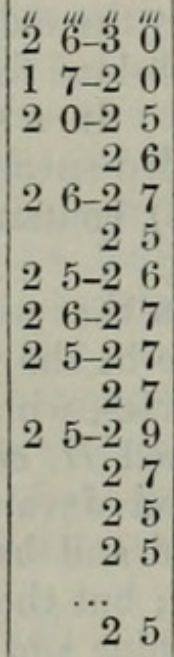 & $\begin{array}{rr}21 & -25 \\
21 & -25 \\
16 & -15 \\
16 & -20 \\
18 & -20 \\
19 & -21 \\
19 \\
18-19 \\
18-19 \\
17-20 \\
18-20 \\
18 \\
21 \\
20 \\
19 \\
12 \\
20 \frac{1}{2}\end{array}$ & $\begin{array}{l}\frac{11}{2}-8 \\
-6 \frac{1}{2} \\
\ldots \\
\ldots\end{array}$ & $\begin{array}{c}7 \\
\ldots \\
\ldots \\
\ldots \\
\ldots \\
\ldots \\
\ldots \\
\ldots\end{array}$ & $\begin{array}{l}8 \frac{1}{2} \\
\cdots \\
\cdots \\
\cdots \\
\cdots \\
\cdots \\
\cdots \\
\cdots \\
\cdots \\
\cdots \\
\cdots \\
\cdots \\
\cdots \\
\cdots \\
\cdots \\
\cdots\end{array}$ & 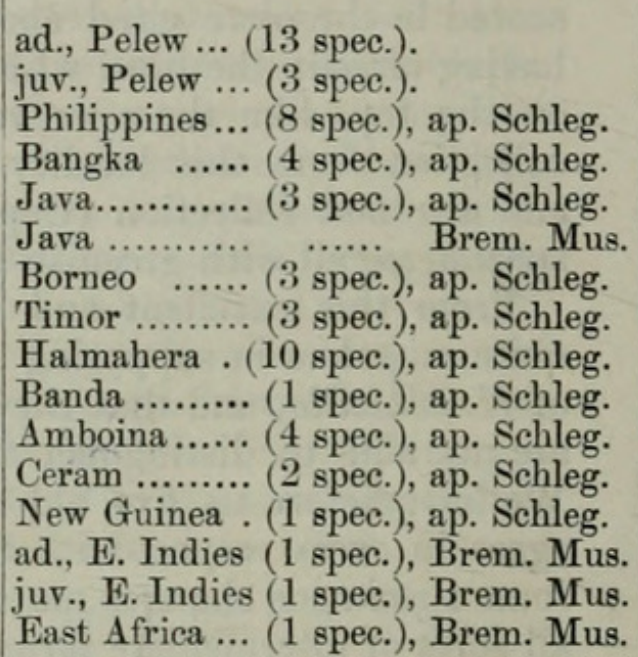 \\
\hline
\end{tabular}

6. Myzomela rubratra, Less.; Hartl. P. Z. S. 1867, p. 829; H. \& F. ibid. 1868 , pp. 5 and 116.

Six specimens from the Pelews (Capts. Heinsohn and Peters), and eight from Uap (Kubary). Amongst the latter there are three specimens, marked by Mr. Kubary as females ("breeding-season"), which agree with the description of the young bird given by us (P. Z. S. 1868, p. 5). They are of a dark olive-brown, intermixed more or less with red feathers; in some nearly the whole head and underparts are red, which shows these variations to be clearly signs of imaturity. Probably the adult female in full dress does not differ from the male.

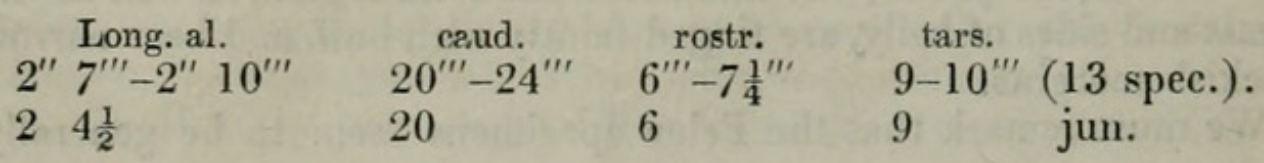


"Umel" of the natives (Kubary).

Mr. Kubary has also sent a nest, said to be of this species. It has a cup-shaped form, is deeper than broad, and was placed between the furcation of a small twig. It consists of stems of grass connected with cobwebs, and is somewhat loosely constructed.

A very interesting account of this species on Ualan is given by v. Kittlitz (Denkwürdigkeiten einer Reise, \&c. i. 1858, pp. 364 \& $381)$.

7. Psamathia anne, Hartl. \& Finsch, P. Z. S. 1868, pp. 5 $\& 116$.

One specimen from Pelew (Capt. Heinsohn).

8. Zosterops Semperi, Hartl.; H. \& F., P.Z. S. 1868, p. 117.

Two specimens from the Pelew Islands (Capt. Heinsohn). In one, the chin and throat are rather of a sulphur-yellow. The nearest ally is $Z$. meyeni, Bp., which is smaller, and has a narrow black loreal stripe, and a yellow rump.

\section{Zosterops hypolais, n. sp.}

Minor. Supra in fundo dilute cinerascente pallido virescente lavata, subtus pallide favescens, pectore abdomineque cinerascenti-sordidulis; subalaribus albis; subcaudalibus dilute favis ; remigibus et rectricibus nigricantibus, dorsi colore marginatis; annulo periophthalmico minus distincto; pedibus plumbeonigricantibus.

Upper parts of a pale greyish green, throat and under tail-coverts a pure but very pale whitish yellow; breast and abdomen of a mixed pale grey and pale yellow; wing- and tail-feathers pale blackish, margined with the greenish colour of the back; under wing-coverts and inner margins of remiges white; eye-ring indistinct; beak fuscous, the under mandible paler, except at the tip; feet plumbeous.

Long. tot. c. $4^{\prime \prime}$, rostr. a fr. $5^{\prime \prime \prime}$, al. $26^{\prime \prime \prime}$, caud. $18^{\prime \prime \prime}$, tars. $9^{\prime \prime \prime}$.

Mr. Kubary has sent three specimens of this interesting new Zosterops from the island of Uap, which agree with each other. The absence of a distinct white eye-ring is the principal feature of this dull-coloured species. $\boldsymbol{Z}$. conspicillata, Kittl., from Guaham, is its nearest ally, but is distinguished by the white front and eye-ring.

\section{Zosterops oleaginea, n. sp.}

Major. Tota oleagineo-virescens, subtus parum dilutior, nonnihil flavo-fulvescente lavata; regione parotica nigricante; annulo periophthalmico niveo; remigibus et rectricibus fusco-nigricantibus, dorsi colore limbatis; rostro infuscato, mandibula flavida, apice fusca; pedibus pallidis.

General colour a deep oil-green, with a decided fulvous hue; underparts a little paler, and a little more yellowish; eye-ring satin-white; ears blackish; upper and under tail-coverts with a slight rufous tinge ; wing- and tail-feathers blackish, with oil-green 
margins; under wing-coverts whitish grey; beak fulvous, under mandible, except at the tip, yellowish ; feet pale, probably yellow ; iris reddish white.

Long. tot. circa $4^{\prime \prime} 7^{\prime \prime \prime}$, rostr. a fr. $6 \frac{1}{2} \prime \prime \prime$, al. $2^{\prime \prime} 7^{\prime \prime \prime}$, caud. $1^{\prime \prime} 8^{\prime \prime \prime}$, tars. $10^{\prime \prime \prime}$.

The colouring of this typical species of Zosterops is quite unique, and does not resemble that of any other.

The collection contains three specimens, male and female, discovered and sent by Mr. Kubary from the island of Uap.

\section{Zosterops finschi, Hartl.}

Tephras finschi, Hartl. P. Z.S. 1868, p. 6, pl. 3, and ibid. p. 117.

One specimen from Pelew (Capt. Heinsohn).

On comparing again this curious bird with the allied Zosteropine members, we have come to the conclusion that Tephras can be considered only a subgeneric division of Zosterops. The wings, having the first quill-feather spurious, are the same as in true $Z$ osterops, the bill corresponds with that of others (for instance, $Z$. oleaginea, which also has a somewhat rotundate tail). The want of a white eye-ring is the only point by which Tephras could be separated.

A near ally seems to be $\boldsymbol{Z}$. cinerea, Kittl., from Ualan.

12. Turdus obscurus, Gmel. ; Finsch, Journ. of Orn. 1863, p. 34.

Turdus pallens, Pall.

Turdus pallidus, Naum. (not Gmel.).

One specimen from the Pelews (Capt. Heinsoln), where this species has not yet been recorded.

\section{Rhipidura versicolor, n. sp.}

Supra fusca; fronte et sincipite late rufis; gula alba, infra nigrocircumdata; pectore albido nigroque maculato; abdomine pallidius fusco, medio albicante; tectricibus cauda superioribus subcaudalibusque rufis; remigibus fusco-nigricantibus, tectricibus alarum dorso concoloribus; subalaribus albidis; rectricibus obscure fuscis, late albo terminatis, 4 mediis basi rufis; rostro et pedibus fuscis.

Upper parts a rich brown with a slight reddish tinge; forehead bright rufous; upper and under tail-coverts rufous; throat white, margined underneath by an irregular jugular band of pure black; pectoral plumes black, broadly margined with yellowish white; middle of abdomen whitish, sides of a paler olive-brown; under wing-coverts whitish; wing-feathers blackish brown; tail-feathers brownish black, all largely tipped with white, the four middle ones rufous at the base, the white terminal spots becoming smaller towards the middle; beak fuscous, the under mandible paler except at the tip ; feet fuscous.

Long. tot. circa $5^{\prime \prime} 9^{\prime \prime \prime}$, rostr. a fr. $4 \frac{1}{2}{ }^{\prime \prime \prime}$, al. $2^{\prime \prime} 6^{\prime \prime \prime}$, caud. $3^{\prime \prime} 3^{\prime \prime \prime}$, tarsi $8^{\prime \prime \prime}$. 
Three specimens, collected by Mr. Kubary at the island of Uap. This typical new species resembles much $R h$. lepida, nob. (from Pelew), and $R h$. torrida, Wall., but may be distinguished at once from both by its tail-feathers being largely tipped with white.

"Atabrue' of the natives" (Kubary).

A nest sent by Mr. Kubary in form and structure resembles much that of Rh. nebulosa, as described by us (Orn. Centr. Polyn. p. 87).

14. Myiagra erythrops, H. \& F., P. Z. S. 1868, pp. 6 \& 117.

One specimen from the Pelews (Capt. Heinsohn), not different from the specimens already received. In this specimen not only the front, but also the forehead to the anterior eye-margin is of a vivid rufous.

15. Monarches godeffroyi, Hartl., P. Z. S. 1867, p. 829, pl. xxxviii.

Eleven specimens in the three different plumages as figured, all collected by Mr. Kubary on the island of Uap.

Three specimens, white beneath and above, with black head, quills, and tail, agree with the description of the old bird and the figure in front. These are marked as males by the collector, and seem to represent the full-plumaged bird.

Two specimens, marked as female, have a broad white collar round the neck; the remainder of the under-surface is black, like the specimen represented in the middle figure, but the mantle is throughout black.

Besides, there are six specimens in the fulvous plumage, like the figure in the background. Our description of this stage being some. what short and imperfect, we thiuk it necessary to give a more complete one, as follows :-

Supra cineraceo-rufescens; pileo et nucha sordide cinereis, uropygio et supracaudalibus dilute rufescentibus; alarum tectricibus remigibusque fuscis, rufo marginatis; subcaudalibus et subalaribus albido-rufescentibus; subtus dilute rufescens, gula et abdomine medio magis albicantibus; oculis pallido rufo circumdatis; rectricibus fusco-nigricantibus, macula apicali alba, extimce latius albo terminatce pogonio externo pallide rufulo; rostro fuscescente, basi pallido; pedibus nigricantibus.

Back rufescent grey; head above dark cinereous, sides of head rufescent ; a pale fulvous ring round the eye ; posterior part of back and upper tail-coverts light rufous; wing-coverts, tertiaries, and scapularies dark fuscous, with broad rufous margins; in the primaries these margins are reduced to an almost imperceptible narrowness ; tail-feathers dark fuscous, with a white terminal spot; in the outer this white spot is much larger, and the external vane is pale reddish; underparts light rufous; throat and middle of abdomen rather whitish; under wing- and under tail-coverts whitish with a more or less rufescent tinge; feet bluish; beak brownish, pale at the base; eyes black.

Proc. Zool. Soc.-1872, No. VII. 
The rufous colour of the underparts varies in intensity, being in some specimens paler and mixed with whitish on the middle of vent, in others uniform cinnamon-rufous or dark rufous.

According to $\mathrm{Mr}$. Kubary, who notes these specimens as males, they belong to a different species; but we are quite sure that they are really the young of $M$. godeffroyi. There is one specimen which shows undoubted signs of change from the red plumage into the black, having the head here and there intermixed with black feathers; the middle tail-feathers are already black; and the cinnamon of the rump and upper tail-coverts is varied with white.

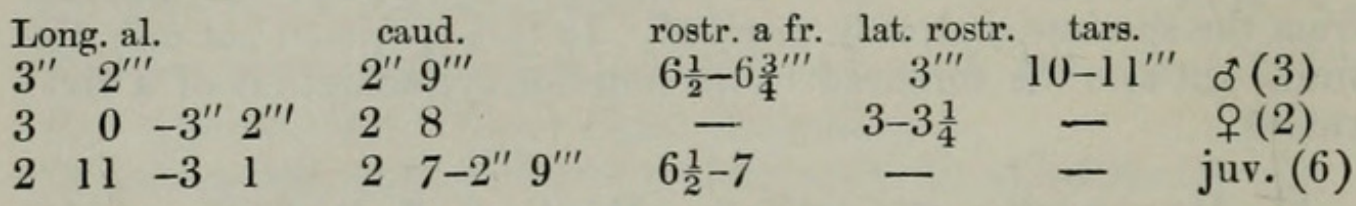

The black and white bird is the "Gigi" of the natives; the young rufous bird is called "Golai."

\section{Campephaga nesiotis, n. sp.}

Jun. Supra rufescenti-fusco fulvoque sordide varia; pileo nuchaque cinerascentibus; superciliis rufis; uropygio et supracaudalibus dilute rufo fuscoque transversim notatis; gastraeo dilute cinnamomeo-rufo, pectore fasciolis rarioribus strictissimis nigris notato; tectricibus alarum minoribus dorso concoloribus; majoribus, remigibus tertiariis scapularibusque fusco-nigricantibus, late rufo marginatis, majorum limbo externo strictissimo areaque majore pogonii interni rufis; subalaribus unicoloribus rufis; rectricibus duabus inlermediis fuscis apice fulvo marginatis, lateralibus fuscescenti-nigris macula apicali majore fulva, extima pogonio externo fulvo, nigro murginato; rostro et pedibus nigris.

Long. tot. circa $9 \frac{1}{2} "$, rostri $10 \frac{1}{2}{ }^{\prime \prime \prime}$, alæ $4^{\prime \prime} 5^{\prime \prime \prime}$, caudæ $3^{\prime \prime} 10^{\prime \prime \prime}$, tarsi $12^{\prime \prime \prime}$.

Back rich brown, indistinctly mottled with fulvous; head above and nape darker and more greyish; evebrows and frontal plumes rufous ; posterior part of back and upper tail-coverts transversely variegated with rufous and dark brown ; beneath light rufescent or fulvous, some narrow black bands on the breast; under wingcoverts and under tail-coverts of a uniform light rufous ; wing-coverts like the back; scapulars and smaller wing-feathers blackish, with broad rufous margins, primaries with a very narrow rufous external margin, the inner vanes of all from the base with a larger rufous marginal space; the two middle tail-feathers brown, margined with rufous at the tip, the lateral ones blackish with a larger fulvous terminal spot, the external one black with a very broad apical part and a narrow longitudinal band on the outer vane light rufous; bill and feet black.

Two specimens from the island of Uap, discovered and sent by Captain Peters. Both specimens are alike, and in the rufous dress of the young bird or female. The old bird will certainly show a 
quite different coloration, but nevertheless prove to be specifically distinct. At least, all our endeavours to unite these rufous-coloured specimens with any of the known species have been unsuccessful. They have, however, great resemblance to the females of $C$. melcena, Müll., and some other Moluccan species.

" 'Astang' of the natives; very rare" (Kubary).

\section{Campephaga monacha, n. sp.}

Saturate casia; plumis supranasalibus lorisque latius nigris; tectricibus alarum remigibusque nigris, dorsi colore marginatis, his pogonio interno a basi ultra medium latissime albo marginatis; subalaribus indistincte fuscintis; subcaudalibus limbo apicali vix conspicue albidis; rectricibus intermediis óscure casiis, apice late nigris, reliquis nigris, omnibus lihbo apicali albidis; rostro et pedibus nigris.

Jun. Supra sordide et obsolete griseo-fuscescens, hinc inde nigro fulvoque transversim notata; subtus fulva, maculis nigris subtriquetris rarius variegata; gutture immaculato; remigibus nigricantibus, dilute rufescente marginatis; rectricibus mediis sordide rufulis, lateralibus fusco-nigricantibus, apice margineque dilute rufescentibus; subalaribus et subcaudalibus late et dilute fulvis, his striis scaparum rarioribus nigris; rostro et pedibus nigricantibus.

Long. tot. circa $8^{\prime \prime} 6^{\prime \prime \prime}$, rostri a fr. $7 \frac{1}{2}{ }^{\prime \prime \prime}$, al. $3^{\prime \prime} 8^{\prime \prime \prime}$, caud. $3^{\prime \prime}$, tarsi $9 \frac{1}{2}^{\prime \prime \prime}$.

$A d$. All over of a dark bluish grey ; lores and a small frontal band velvet-black; throat somewhat darker ; wing-feathers black, coverts and smaller remiges with a broad margin of bluish grey; this margin is very narrow on the greater ones, and does not extend to the tip; an oblique white belt on the inner vanes; intermediate tailfeathers dark bluish grey with broad black tips, lateral ones black, all with a narrow whitish terminal margin; under wing-coverts indistinctly barred; under tail-coverts bluish grey, with a very narrow apical margin of white; bill and feet black.

No species of the widely distributed group of Campephagine birds has been vet recorded from the Pelew Islands. For the discovery of this interesting new species we are indebted to Capts. Heiissohn and Peters; the first-named gentleman sent the young, the last. named the old bird, from the Pelews.

This species comes nearest to C. morio, Temm., from Celebes; but the latter is larger, and has the sides of head, chin, and throat black.

18. Artamus leucorhynchus (Gmel.); H. \& F., P. Z. S. 1868, p. 116.

One specimen agreeing with the one mentioned by us from the Pelews; but the bill is of a delicate light cobalt-blue, with dark tip.

19. Rectes tenebrosus, H. \& F., P. Z. S. 1868, pp. 6 \& 117. Two specimens from the Peiew Islands (Capt. Peters) agreeing 
with the type-specimen described by us. We are now of opinion that this plumage is not that of the young bird, but of the old.

$$
\begin{array}{cccc}
\text { Long. al. } & \text { caud. } & \text { rostr. } & \text { tars. } \\
3^{\prime \prime} 6^{\prime \prime}-3^{\prime \prime} 8^{\prime \prime \prime} & 2^{\prime \prime} 6^{\prime \prime \prime}-2^{\prime \prime} 7^{\prime \prime \prime} & 8^{\prime \prime \prime} & 12^{\prime \prime \prime}
\end{array}
$$

20. Calornis kittlitzi, F. \& H.; Hartl. P. Z. S. 1867 , p. 830 ; H. \& F. ibid. 1868, pp. 7 \& 117.

Three specimens from Mackenzie Island (Capt. Peters), and two from Uap (Kubary), where this species had not been previously observed.

One specimen, determined as female by Mr. Kubary, agrees in colour with the old male; a young one (marked as male) is of a uniform sooty brown, with slight metallic reflexions on the upper parts. "Iris yellow" (Kubary).

The dimensions vary very much in this species, but do not equal those of the allied C. corvina, Kittl., from Ualan.

Long al. caud. $4^{\prime \prime} 4^{\prime \prime \prime}-4^{\prime \prime} 10^{\prime \prime \prime}$

$\begin{array}{llll}4 & 7 & -5 & 0\end{array}$

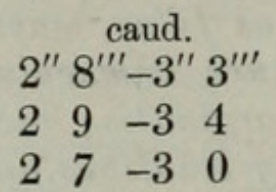

rostr.
$8 \frac{1}{2}-9 \frac{1}{2}^{\prime \prime \prime}$
$8 \frac{1}{2}-9$
$9-9 \frac{1}{2}$

tars.
$12-13^{\prime \prime}$
$13 \frac{1}{2}-14$
13
'(Pelew, 9 spec.)
(Mackenzie, 9spec.)

$\begin{array}{lllllllllllll}4 & 6 & -4 & 8 & 2 & 7 & -3 & 0 & 9 & -9 \frac{1}{2} & 13 & -14 & \text { (Uap, } 2 \text { spec.) }\end{array}$

21. Cuculus canorus, L.; Swinh. P.Z. S. 1863, p. 264.

Cuculus telephonus, Heine, jr., Journ. f. Orn. 1863, p. 352.

An old specimen from the Pelew Islands (Capt. Heinsohn) proves to be specifically identical with our common Cuckoo. In comparing this specimen with an old male from Germany, we can notice only that the white underparts are tinged very faintly with yellowish, and that the dark cross bands on the under tail-coverts are less marked. The zigzags on the anal region are absent.

I have also seen in the Leyden collection a specimen labelled "Luçon," which was entirely similar to the European bird.

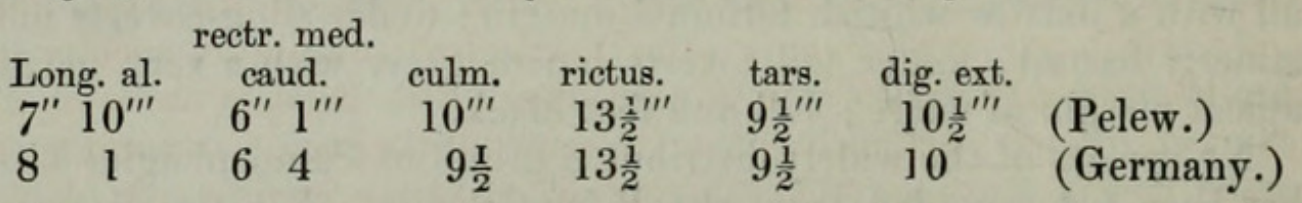

The occurrence of the Common Cuckoo in the Pelews is a new fact in the geographical distribution of this species.

22. Cuculus striatus, Drapiez; Schleg. Mus. P.-B. Cuculi, p. 7.

Cuculus himalayanus, Swirh. P. Z. S. 1863, p. 265.

Three specimens from the Pelews (Capts. Heinsohn and Peters) are nearly in full plumage-one changing the feathers of the upper parts from brown into dark slate, and two young ones in the dark rufous plumage barred with dark. I have for comparison only an old specimen from Amboina at hand, which shows no difference.

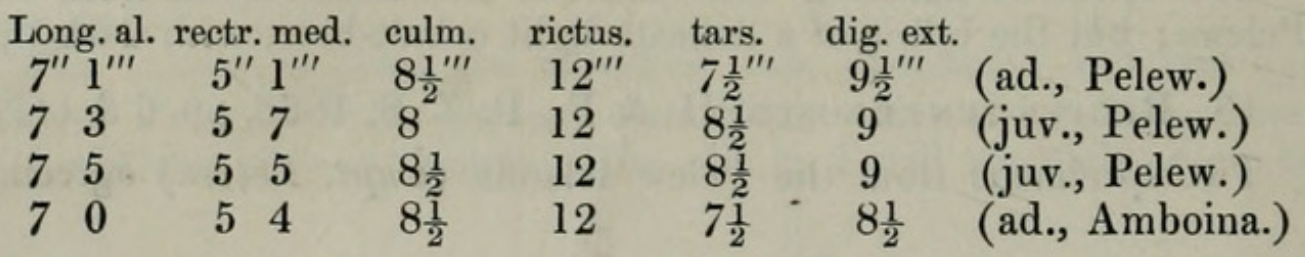


New to the Pelews.

Peale's $C$. fucatus, from Mangsi, in the Sooloo archipelago (C. tenuirostris, Cass. Atl. pl. 21.f. 1) belongs not to this species, but to the smaller C. micropterus, Bl. (Schleg. Cuculi, p. $12=C$. tenuirostris, Cab. \& Heine, Mus. Hein. p. 39, nec C. tenuirostris, Müll.).

\section{Ptilinopus pelewensis, H. \& F., P. Z. S. 1868, p. 7.}

Six specimens from the Pelews (Capts. Peters and Heinsohn), agreeing exactly with the description given by us, as cited above. The outermost secondaries (the so-called tertiaries) have an acute angulated shaft-end spot of brilliant violet-blue ; the under tail-coverts are bright purplish red, with narrow bright orange edgings ; feet dark blood-red; bill lead-coloured, with greenish-white tips.

Young bird. Green, with narrow yellow edgings on the tectrices and scapulars; head above also green, with a pale yellow supercilium ; underparts dirty olive-green; vent, anal region, and under tail-coverts straw-yellow; on the vent some orange feathers; tail green, with a whitish patch on the inner webs above the end, forming an irregular cross band; feet and bill dark.

$\begin{array}{llcl}\text { Long. al. } & \text { caud. } & \text { rostr. } \\ 4^{\prime \prime} 10^{\prime \prime \prime}-5^{\prime \prime} & 2^{\prime \prime} 7^{\prime \prime \prime} & 6-6 \frac{1}{2}{ }^{\prime \prime \prime} & \text { (5 spec.) } \\ 4 \quad 8 & 2 & 4 & 6\end{array}$

24. Carpophaga oceanica (Less.); Hartl. P. Z. S. 1867, p. 830 .

Carpophaga pacifica, H. \& F., P. Z. S. 1868, p. 7.

One old specimen from the Pelew group (Capt. Heinsohn), agreeing exactly with the figure of Kittlitz (Kupfert. 33. f. 1), who observed the bird on the island of Ualan (Denkwürdigk. i. p. 377). We must remark that none of the specimens we have examined from this group show the ferruginous-vinaceous tinge on the crop and breast, although this tinge is sometimes also wanting in specimens from the Navigator and Friendly groups. But, what is more significant, the Pelew specimens have the grey on the head and hind neck much darker; besides, the under wing-coverts are brownish black, whereas in $C$. pacifica from Central Polynesia they are of a dark ashy grey. The chestnut-red on the vent, anus, and under tail-coverts appears to be also much darker in Pelew specimens. Therefore we incline to believe that the Carpophaga from the north-western Pacific Islands may form a distinct species-the Columba oceanica of Lesson; but before we can settle this question with certainty we must wait for further examination, based upon more specimens.

25. Phlegenas canifrons, n. sp.

Supra olivaceo-virescens, nitore nonnullo metallico; occipite, collo postico et interscapulio late ferrugineis; fronte et sincipite, capitis, colli et pectoris lateribus cinereis; gula et collo antico magis albicantibus; alis dorso concoloribus, area majore scapulari pulchre violaceo-purpurascente; remigibus fuscis, pogonio 
interno a basi inde pro majore parte rufis; subalaribus rufis; abdomine sordide yriseo-fuscescente; rectricibus intermediis dorso concoloribus, roliquis intense fuscis, ante apicem rufescentem obscurius adumbratis; rostro nigricante; pedibus rubris.

Back, wings, upper tail coverts, and median tail-feathers of a rich olive-brouze green ; forehead, top and sides of the head, sides of neck, and breast plumbeous grey; throat and fore neck vinaceous whitish; posterior part of head and neck and interscapulars of a rich rufous ; a large shoulder-spot purplish violet; abdomes: of a dirty and dark greyish brown ; wing feathers blackish, the inner vaine from the base for more than two thirds rufous, which colour does not quite reach to the shaft, the outer vane with a reddish tinge for the same extent; under wing-coverts rufous; lateral tail-feathers fuscous, somewhat darker before the slightly rufescent tip; the middle ones more like the back, beak blackish; feet red; nails brown.

Long. tot. circa $10^{\prime \prime}$, rostr. a fr. $8 \frac{1}{2} " \prime$, al. $4^{\prime \prime} 2^{\prime \prime \prime}$, caud. $2^{\prime \prime} 9^{\prime \prime \prime}$, tars. $1^{\prime \prime}$.

A single specimen, collecteć by Capt. Peters on the Pelews, and apparently in full plumage. This is a very remarkable species, distinguished by the grey of the forehead and along the sides of the neck, and by the cinnamon-rufous of the occiput and hind neck, besides by its small size.

\section{Phleganas yapensis, $n$. sp.}

Jun. av. Supra sordide rufa; pileo unicolori; dorsi et uropygii plumis, alarum tectricibus, scapularibus, remigibus tertiariis et supracaudalibus fuscis, large rufo marginatis; pectore cinerascente rufoque nebuloso; abdominis plumis fuscescentibus, apice rufescentibus; subalarbus obscure fusco rufoque variis; subcauralibus fusco-nigricantibus ; area scapulari obscure violaceopurpurascente; remigibus majoribus fuscis, margine apicali ex/imo rufo; rectricibus obscure rufis, ante apicem late nigris; rostro (ut videtur) flavido, pedibus nigricantibus.

Juv. Dorso nitore virescenti-metallico valde conspicuo; alarum tectricibus scapularibusque minus virescentibus; capite toto rufo; pectore et abdomine obsolete rufescentilus, illo nitore nonnullo subviolacescente lavato; subalaribus et subcaudalibus obscure rufis; cauda ut in ave modo descripta; rostro nigricante.

Long. tot. circa $10^{\prime \prime}$, rostri $7 \frac{1}{2}{ }^{\prime \prime \prime}$, al. $5^{\prime \prime} 3^{\prime \prime \prime}$, caud. $3^{\prime \prime} 6^{\prime \prime \prime}$, tars. $13^{\prime \prime \prime}$.

Two immature specimens. The elder bird (marked as male) has the upper and lateral part of the head light rufous; the feathers of the whole back, the wing-coverts, the scapularies, and tertiaries are of a dark brown, with broad margins of pale rufous ; a large shoulderspot purplish violet, mixed with pale rufous; primaries of a uniform blackish brown; tail-feathers light rufous, with a broad black band before the tip; the throat seems to be of a dirty rufescent colour ; the feathers of the breast pale bluish grey margined with rufous, those of the abdomen and under tail-coverts blackish, variegated with obsolete rufescent shades; under wing-coverts of an obscure mixture of 
black and rufous; bill yellowish, darker round the base; feet plumbeous, with pale nails.

A still younger bird (marked as female) is rather differently coloured, the back being of a dark olivaceous green, with bronze reflexions; wing-coverts, tertiaries, and scapularies also more greenish, with broad rufous margins; no purplish shoulder-spot; the whole head light rufous; breast and abdomen of an indistinct olivaceousrufescent hue; under wing-coverts rufous; tail as in the elder bird.

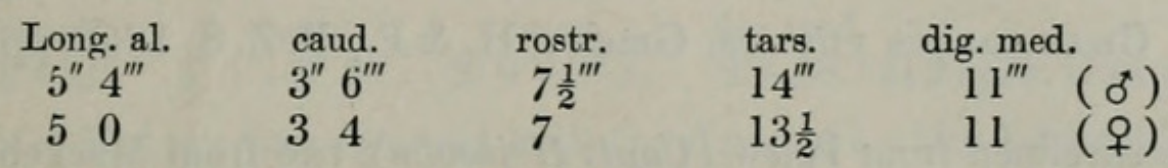

Mr. Kubary has sent only these two specimens, which unfortunately represent two different stages of the imperfect bird. The one marked as female shows some resemblance to the rare Pigeon from the Mariannes named by Bonaparte Pampusana rousseaui. Although neither specimen is in full plumage, we do not hesitate to introduce the species as new, as there are certain signs which lead us to expect that the old bird will prove its specific distinctness.

"The 'Arolit,' as the species is called by natives, is the only Pigeon inhabiting the island of Uap, and is of very rare occurrence there. The Arolit lives in the interior of the wood, far from the settlements of the natives" (Kubary).

27. Megapodius Senex, Hartl. P. Z. S. 1867 , p. 830 ; H. \& F. ibid. 1868, p. 7 .

Two old specimens from Pelew (Capts. Peters and Heinsohn), and the very young in the first plumage. This resembles very much the "Alecthelia durvillei," Less. (Voy. Coq. pl. 37), which is the young of $M$. freycinet $i$; but the head and upper parts are dark olivebrown, the rump and caudal down red-brown; chin and throat yellowish; feet dark reddish brown.

The Megrpodius senex is an excellent species, distinguished at once by the very small vivid-yellow bill, the brownish-grey crested cap, the yellowish or brownish legs, and black toes.

\begin{tabular}{|c|c|c|c|c|c|c|}
\hline $\begin{array}{c}\text { Long al. } \\
6^{\prime \prime} 8^{\prime \prime \prime} \\
64\end{array}$ & $\begin{array}{l}\text { caud. } \\
2^{\prime \prime} 2^{\prime \prime \prime} \\
1\end{array}$ & $\begin{array}{c}\text { rostr. } \\
7^{\prime \prime \prime}\end{array}$ & $\begin{array}{l}\text { rict. } \\
11^{\prime \prime \prime} \\
1 ?\end{array}$ & $\begin{array}{l}\text { tars. } \\
2^{\prime \prime} 2^{\prime \prime \prime} \\
2\end{array}$ & $\begin{array}{c}\text { dig. med. } \\
17^{\prime \prime \prime} \\
18\end{array}$ & $\begin{array}{l}\text { ung. } \\
8^{\prime \prime \prime} \\
8\end{array}$ \\
\hline
\end{tabular}

The collection contains also one egg of this species, which, in size, form, and coloration, is almost nearly undistinguishable from that of M. pritchardi, Gray. Length $2^{\prime \prime} 9^{\prime \prime \prime}$, breadth $1^{\prime \prime} 8^{\prime \prime \prime}$.

\section{Gallus bankiva, Temm.}

Capt. Peters has sent from the Pelews a single female specimen, but unfortunately he does not state whether the Jungle-fowl lives in a wild state on the islands, or as in Uap, as Mr. Kubary remarks (in litt.), only in a half-wild state.

The specimen agrees entirely with a hen from Sumatra; only the 
golden-yellow feathers on the hind neck are paler; besides, it is somewhat paler in colour.

$$
\begin{array}{cccccc}
\text { Long; al. } & \text { caud. } & \text { rostr. } & \text { rict. } & \text { tars. } & \text { dig. med. } \\
7^{\prime \prime} & 5^{\prime \prime} 1^{\prime \prime \prime} & 6 \frac{1^{\prime \prime \prime}}{2} & 13^{\prime \prime \prime} & 26^{\prime \prime \prime} & 18^{\prime \prime \prime}
\end{array}
$$

"Mallett" of the natives.

Wild Jungle-fowls have not yet been recorded from the Pelews. $\mathrm{Mr}$. Kubary notices the very rare occurrence of half-wild fowls in the interior of Uap.

29. Charadrius fulvus, Gmel.; H. \& F., P. Z.S. 1868 , pp. 8 $\& 117$.

One specimen from Pelew (Capt. Heinsohn), two from Mackenzie (Capt. Peters), and three from Uap (Kubary).

As regards the specific distinctness of this species from Ch. pluvialis we must refer to what we have said (Journ. f. Orn. 1870, p. 139). The specimens contained in this collection confirm our opinion, as will be shown in the following measurements :-

\begin{tabular}{|c|c|c|c|c|c|}
\hline $\begin{array}{l}\text { Long. al. } \\
{ }^{\prime \prime} 0^{\prime \prime \prime}-6^{\prime \prime} 4^{\prime \prime \prime}\end{array}$ & $\begin{array}{c}\text { caud. } \\
2^{\prime \prime} 0^{\prime \prime \prime}-2^{\prime \prime} \\
3^{\prime \prime \prime}\end{array}$ & $\begin{array}{l}\text { rostr. } \\
10-11^{\prime \prime \prime}\end{array}$ & $\begin{array}{l}\text { tars. } \\
18-20^{\prime \prime}\end{array}$ & $9-11^{\prime \prime}$ & $10-11^{\prime \prime \prime}$ \\
\hline $4-67$ & $\begin{array}{rrrr}2 & 1 & -2 & 4 \\
& 1\end{array}$ & $\begin{array}{r}10-11 \\
10\end{array}$ & $\begin{array}{l}21 \\
19\end{array}$ & $10-12$ & 12 (Mackenzie.) \\
\hline
\end{tabular}

"“ Kuling' of the natives" (Kubary).

30. Strepsilas interpres (L.); Hartl, P. Z. S. 1868, p. 831; H. \& F. ibid. 1868, p. 8.

Specimens from Pelew (Capt.Heinsohn), Mackenzie (Capt. Peters), and Uap (Kubary). Some of them still show signs of the summer plumage; others are in the perfect winter dress.

"“ 'Kuling' of the natives” (Kubary).

31. Ardea Sacra, Gmel.; Hartl. P. Z. S. 1867, p. 831 ; H. \& F. ibid, 1868, pp. 8 \& 118.

Fourteen specimens from the Pelews (Capt. Peters) and Uap (Kubary); amongst them eight slate-coloured specimens, five white ones, and one in the reddish-brown plumage of the young bird.

The great variation in colour and size which this species exhibits we have already explained several times, especially in our paper on the birds of the Tonga Islands (Journ. f. Orn. 1870, p. 136). We have also stated that the white birds are by no means young ones. The large collection before us confirms all these statements fully.

There are two slate-coloured females, one with a narrow interrupted, the other with a broad well-defined white stripe on chin and throat, shot in December 1870, on which Mr. Kubary notices on the label, " ovaries with full-developed eggs, some more than 2 "' long ;" whereas he remarks on a uniform white male shot at the same time, "testicles very large." This latter male specimen is in full plumage, with full-grown scapular feathers, whereas one of the slate-coloured females shows still some moulting-feathers. 
Two white females, shot in September, have the wings mixed more or less with slate-coloured feathers.

One female, shot in December, is strongly in moult, especially on the neck, where the new dark slate-coloured feathers predominate over the old reddish-brown feathers, the remains of the young plumage.

In one slate-coloured male, shot in December, there is only a small white spot on the middle of the throat. Another male, shot in September, has no white feathers at all. The dimensions are, as usual, very variable.

Long. al. rostr. tars. dig. med. $9^{\prime \prime} 9^{\prime \prime \prime}-11^{\prime \prime} \quad 3^{\prime \prime} 1^{\prime \prime \prime}-3^{\prime \prime} 7^{\prime \prime \prime} \quad 2^{\prime \prime} 9^{\prime \prime \prime}-3^{\prime \prime} 3^{\prime \prime \prime} \quad 23-38^{\prime \prime}$ (12 spec. from Uap.)

Mr. Kubary has observed both the dark and white bird fishing in company. These birds frequent chiefly the lagoons, and roost on the mangroves near the shore. The nest consists only of an excavation of the ground, bordered by stems of grass (?). The natives distinguish the black bird under the name "Khau," the white as "Wunensy," and believe the different colorations to be sexual.

Concerning this species see also v. Kittlitz (Denkwürd. einer Reise, i. p. 368), who observed the bird on Ualan.

\section{Ardea sinensis, Gmel.}

Ardea lepida, Horsf.

Six specimens from Uap, collected (in the beginning of October) by Mr. Kubary and Capt. Peters.

"Irides yellow" (Kubary).

They agree in every respect with specimens from Java.

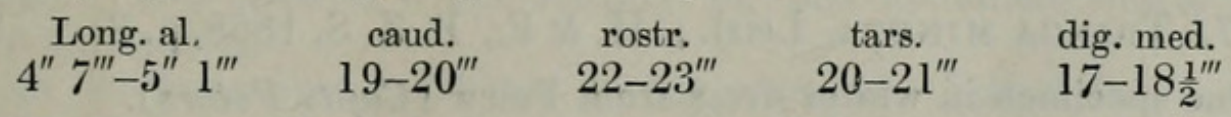

The Western Carolines are a new locality for this widely-distributed species.

The "Thogil," as the bird is called by the natives, resorts chiefly to the mangrove-scrub on the shore.

33. Nycticorax griseus (L.).

One specimen from Uap (Kubary) in the spotted plumage of the young.

Mr. Kubary notices this species as a very rare visitor on the island of Uap during the north-east monsoon.

$\begin{array}{ccccccc}\text { Long. al. } & \text { caud. } & \text { rostr. } & \text { rict. } & \text { tars. } & \text { tib. } & \text { dig. med. } \\ 10^{\prime \prime} 6^{\prime \prime \prime} & 3^{\prime \prime} 9^{\prime \prime \prime} & 2^{\prime \prime} 5^{\prime \prime \prime} & 3^{\prime \prime} 4^{\prime \prime \prime} & 2^{\prime \prime} 5^{\prime \prime \prime} & 6^{\prime \prime \prime} & 2^{\prime \prime} \\ 4^{\prime \prime \prime}\end{array}$

"'The natives call this 'Orror ;' it is very rare" (Kubary).

34. Nycticorax manillensis, Vig.; Schleg. Mus. P.-B. Ardece, p. 60 .

Nycticorax caledonicus, H. \& F., P. Z. S. 1868, p. 117 (Pelew).

Two old and a young specimen from the Pelew Islands (Capt. Heinsohn). 
In our last list of the birds of the Pelew Islands we have inserted this species wrongly as $N$. caledonicus, from which it seems to differ specifically in having the back, shoulders, and wing-coverts of a much darker cinnamon-castaneous; the neck is also darker. The young birds differ also a good deal from the young of $N$. caledonicus, having the sides of head and the front of the neck brown, each feather with a narrow white shaft-stripe.

Long. al. caud. rostr. rict. tars. tib. dig. med. $10^{\prime \prime} 3^{\prime \prime \prime}-11^{\prime \prime} \quad 3^{\prime \prime} 7^{\prime \prime \prime}-3^{\prime \prime} 11^{\prime \prime \prime} \quad 2^{\prime \prime} 6^{\prime \prime \prime}-2^{\prime \prime} 9^{\prime \prime \prime} \quad 3^{\prime \prime} 4^{\prime \prime \prime}-3^{\prime \prime} 8^{\prime \prime} \quad 3^{\prime \prime}-3^{\prime \prime} 2^{\prime \prime \prime} \quad 12-15^{\prime \prime \prime} \quad 2^{\prime \prime} 4^{\prime \prime \prime}-2^{\prime \prime} 6^{\prime \prime}$

35. Numenius pheopus, Lath. ; Hartl. P. Z. S. 1867, p. 831; H. \& F. ibid. 1868, p. 8.

Two specimens from Uap (Kubary), and one from the Pelews (Capt. Peters).

There is no difference between these and European specimens.

"Lives in great swarms; roosts during night on trees" (Kubary).

36. Tringa acuminata, Horsf.; H. \& F., P. Z. S. 1868, p. 8.

One specimen from Pelew (Capt. Heinsohn), agreeing with the specimen received formerly thence, but smaller. Similar to Australian specimens.

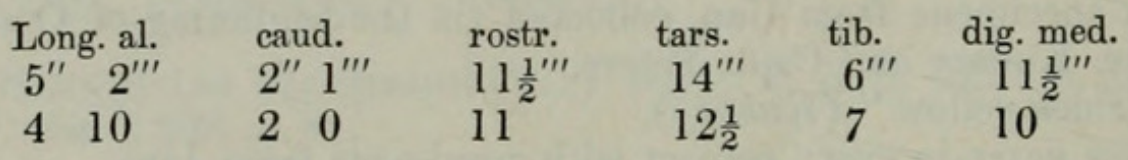

37. Tringa minuta, Leisl.; H. \& F., P. Z. S. 1868, p. 8.

One specimen in winter dress from Pelew (Capt. Peters).

38. Actitis incanus (Gmel.), F. \& H. Orn. Centr. Polyn. p. 182 .

One specimen from Uap (Kubary) in the barred summer plumage.

Not yet recorded from this locality.

$$
\begin{aligned}
& \text { Long. al. caud. rostr. tars. dig. med. } \\
& 6^{\prime \prime} 5^{\prime \prime \prime} \quad 2^{\prime \prime} 5^{\prime \prime \prime} \quad 18^{\prime \prime \prime} \quad 14^{\prime \prime \prime} \quad 11 \frac{1}{2}{ }^{\prime \prime \prime} \text { (Uap.) } \\
& 6^{\prime \prime} 2^{\prime \prime \prime}-6 \quad 8 \quad 2^{\prime \prime} 5^{\prime \prime \prime}-2 \quad 9 \quad 16 \frac{1}{2}-17 \quad 13 \frac{1}{2}-15 \quad 11 \frac{1}{2}-12 \quad \text { (Upolu, } 6 \text { spec.) }
\end{aligned}
$$

Specimens from the Navigators, (Upolu), shot in September by Dr. Gräffe, bear different plumages. One has still the banded summer dress; others have already partially or totally assumed the uniform winter dress. Von Kittlitz observed this species on Ualan, in the Eastern Carolines (Tringa glareola, Denkwürd. i. p. 365).

39. Actitis hypoleucus, L.; H. \& F., P. Z. S. 1868, p. 8.

One specimen from Pelew (Capt. Peters).

40. Rallina fasciata (Raffl.).

Gallinula euryzona, Temm. 
Rallina fasciata, Hartl. P. Z. S. 1867 , p. 831 ; H. \& F. ibid. 1868 , p. 7 .

Three old and one young specimens (Capts. Heinsohn and Peters) from Pelew.

There is no difference whatever between these and specimens from Java and Malacca.

$$
\begin{array}{cccc}
\text { Long. al. } & \text { rostr. } & \text { tars. } & \text { dig. med. } \\
4^{\prime \prime} 5^{\prime \prime \prime}-4^{\prime \prime} 9^{\prime \prime \prime} & 9-9 \frac{1^{\prime \prime \prime}}{2} & 17 \frac{1}{2}-19 \frac{1}{2}{ }^{\prime \prime \prime} & 12-13^{\prime \prime \prime}
\end{array}
$$

41. Rallus pectoralis, Less.

Rallus philippensis, Hartl. P. Z. S. 1867, p. 831.

Rullus pectoralis, H. \& F. ibid. 1868, pp. $8 \& 117$.

Three species from the Pelew Islands (Capts. Heinsohn and Peters). In one the cinnamon cross band on the breast is well marked, in the other barely visible; in a younger one it is wanting totally. In one the black feathers on the hind neck have only white marginal spots; in the other they are barred distinctly with white.

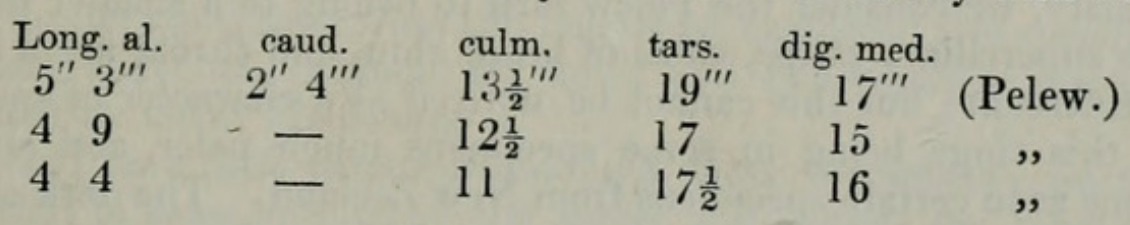

42. Ortygometra quadristrigata, Horsf.; H. \& F., P. Z.S. 1868 , p. 8.

Old and young birds from Pelew (Capt. Peters) and Uap (Kubary). This latter is a new locality for this extremely widely distributed species.

As we have already shown in our work on Central-Polynesian Ornithology (p. 166), specimens from the Navigators' Islands and Java agree in every respect.

The size varies considerably.

"Breeding-season in July and August. The bird, named by the natives 'Bal,' lives in the wooded region, and is here plentiful. It has a loud voice. Its nest is very roughly made in the grass. It is often attacked by the Galufs (Hydrosaurus marmoratus), a species of Lizard about 3 feet long." (Kubary.)

43. Porphyrio melanonotus, Temm., var. pelewensis, nob.

Porphyrio melanotus, H. \& F., P. Z. S. 1868, p. 8 (Pelew); id. ibid. p. 117 .

Four specimens from the Pelew Islands (Capt. Peters), which again prove the smaller size to be a constant character of the race of the Pelew group, as they have the wings and tarsus always considerably shorter. The bill and frontal shield vary as much as in $P$. melanonotus, with which in coloration it agrees perfectly. As in that species, the remiges show more or less a blue shining margin along the outer web; the blue patch on the humerus in some specimens is brighter. If the notice "legs yellow," given formerly by 
one of M. Godeffroy's collectors, should be right, no doubt the Porphyrio of the Pelews would rank as a species; but we doubt this, because, so far as we can judge from the dried skins, the colour of the legs in life is red as in P. melanonotus.

We append for comparison measurements taken from eight specimens from Pelew, and seven of the true $P$. melanonotus from Australia and New Zealand.

\begin{tabular}{|c|c|c|c|c|c|c|c|}
\hline al. & $\begin{array}{l}\text { caud. } \\
2^{\prime \prime} 9^{\prime \prime \prime}-3^{\prime \prime} 2^{\prime \prime \prime}\end{array}$ & $\begin{array}{l}\text { rostr.incl. scut. } \\
2^{\prime \prime} 1^{\prime \prime \prime}-2^{\prime \prime} 8^{\prime \prime \prime}\end{array}$ & $\begin{array}{l}\text { atit. scut. } \\
6 \frac{1}{2}-13^{\prime \prime \prime}\end{array}$ & $\begin{array}{l}\text { rict. } \\
15-17 \frac{1}{2}^{\prime \prime \prime}\end{array}$ & $\begin{array}{c}\text { tars. } \\
2^{\prime \prime} 9^{\prime \prime \prime}-3^{\prime \prime} 0^{\prime \prime \prime}\end{array}$ & $\begin{array}{l}\text { tib. } \\
12-15^{\prime \prime \prime}\end{array}$ & $\begin{array}{l}\text { dig. med. } \\
2^{\prime \prime} 10^{\prime \prime \prime}-3^{\prime \prime} 1^{\prime \prime \prime} \text { * }\end{array}$ \\
\hline $\begin{array}{lll}7 & -10 & 9\end{array}$ & $\begin{array}{llll}3 & 3 & -4 & 1\end{array}$ & $\begin{array}{llll}2 & 3 & -2 & 10\end{array}$ & $8^{2}-11 \frac{1}{2}$ & $16-19^{2}$ & $\begin{array}{llll}3 & 4 & -3 & 9\end{array}$ & $15-22$ & -37 \\
\hline
\end{tabular}

44. Anas superciliosa, Gmel., var. pelewensis, H. \& F.

A. superciliosa, Hartl. P. Z. S. $186 \overline{7}$, p. 832 ; H. \& F. ibid. 1868 , p. 8.

Two specimens from the Pelews (Capt. Heinsohn), which agree perfectly with specimens from New Zealand and Australia, except in their smaller size. Having examined four specimens, which all exhibit this peculiarity, we consider the Pelew bird to belong to a smaller race.

The superciliary stripe, sides of head, chin, and throat are a little more rufescent; but this cannot be noticed as a character of specific value, this tinge being in some specimens much paler, and almost the same as in certain specimens from New Zealand. The dark stripe from the angle of the mouth also varies in intensity, and is nearly altogether wanting in some specimens.

The two last-received specimens show another strange peculiarity, in two or three of the outer secondaries (the so-called tertiaries) having on their dark-brown outer web three or four longitudinal light-brown patches, forming sometimes a broad longitudinal stripe.

A. luzonica, Fras. (P. Z. S. 1839, p. 112), seems to be an excellent species, being described as having head and neck "pallide castanea," and a white cross band on the wings, formed by the white band of the tectrices of the secondaries.

\begin{tabular}{|c|c|c|c|c|c|c|c|}
\hline $\begin{array}{l}\text { al. } \\
" 6 " \text { " }\end{array}$ & $\begin{array}{l}\text { caud. } \\
3^{\prime \prime} 0^{\prime \prime \prime}\end{array}$ & $\begin{array}{l}\text { rostr. } \\
\text { a front. } \\
18-19 \frac{1}{2}{ }^{\prime \prime \prime}\end{array}$ & $\begin{array}{c}\text { Alt. max } \\
\text { ad bas. } \\
6-7^{\prime \prime \prime}\end{array}$ & $\begin{array}{l}\max \text {. } \\
\text { ad apic. } \\
8 \frac{1}{2}-9^{\prime \prime \prime}\end{array}$ & $\begin{array}{l}\text { Long. } \\
\text { tars. } \\
15 \frac{1}{2}-16^{\prime \prime \prime}\end{array}$ & $\begin{array}{l}\text { dig. med. } \\
18-20^{\prime \prime \prime}\end{array}$ & (Pel \\
\hline $\begin{array}{lll}0 & -9 & 6\end{array}$ & $3^{\prime \prime}-3 \quad 4$ & $20 \frac{1}{2}-22$ & $8-8 \frac{1}{2}$ & $9-9 \frac{1}{2}$ & $17-18$ & $21-23$ & $6 \mathrm{ex})$. \\
\hline
\end{tabular}

45. Puffinus dichrous, H. \& F. Orn. Centr. Polyn. p. 244.

Procellaria obscura, Kuhl (nec Gmel., nec auct.), Zool. Beitr. iii. (1820) p. 147 , t. xi. f. 11 (cap.); Temm. Man. d'Orn. ii. (1820), p. 808 (sol. descr.) ; Schleg. Mus. P.-B. Procell. (1863) p. 30 (syn. emend.).

Puffinus opisthomelas, var. minor, Hartl. P. Z. S. 1867, p. 382 ; H. \& F. ibid. 1868, pp. 9 \& 371 ; Finsch, J. f. Orn. 1870, p. 371. ? Puffinus obscurus, Degl. Orn. Europ. ii. p. 366 (syn. excl.); Degl. \& Gerbe, ibid. sec. ed. p. 380 .

? Procellaria obscura, Schleg. \& Pollen, Rech. Faun. Mad.p. 144.

* Pelew.

$\dagger$ Austr., New Zealand. 
Upper parts, including wing and tail, sooty black; this colour extends from the angle of the mouth, bordering the eye beneath, to the middle of the ear-region; the sides of the neck and breast sooty greyish black, tipped narrowly with whitish ; remainder of the underparts white; the central and lateral under tail-coverts sooty black, some of the former very narrowly margined at the apex with white; the anterior central under tail-feathers greyish black, towards the basal half whitish, and with broader white apical margins; under wingcoverts white, along cubitus and manus bordered with sooty-black feathers ; the remiges on the inner web towards the base become paler brownish ; the tectrices of the secondaries very faintly and narrowly tipped with whitish ; there is a slight indication of a white line bordering the eye underneath ; bill hornish, dark brown, tip paler ; inside of tarsus, toes, and membranes pale hornish yellow ; external toe and outside of tarsus dark blackish brown, lighter, into brownish yellow towards the basal half of tarsus; nails blackish; the tail is somewhat cuneate, the tips of the quills reach to about one third from the end of the tail.

In former collections from the Pelews we examined five specimens; this last collection contains six adult and two young examples, all collected by Capt. Heinsohn.

There exists little variation amongst these specimens; we notice only that in some specimens the under tail-coverts are throughout sooty black, in others the anterior lateral under tail-coverts are white at the greater basal portion of the inner web. The very faint whitish tips of the tectrices of the secondaries are in some specimens almost wanting; the sides of the thighs have sometimes a greyish dark mark.

The two nestlings are clothed uniformly with thick greyish-brown down, changing on the middle of the underparts into white. Coloration of feet as in old birds. The young from M'Kean's Island are precisely similar.

Through the kindness of Dr. Cabanis we received the type of our $P$. dichrous from M'Kean's Island (collected by Dr. Gräffe), and now deposited in the Berlin Museum. After a careful comparison, we have not the slightest doubt as to its identity with Pelew specimens. The type specimen of $P$. dichrous, the only one we ever received from the Central Pacific, has a little shorter wings. The anterior lateral under tail-coverts are white on the greater portion of the inner web, just as in some specimens from the Pelews. The coloration is quite the same.

In our previous memoirs on the Pelew birds we took this Petrel, although with some doubt, for $\boldsymbol{P}$. opisthomelas, noticing the considerably smaller size. Having now examined about a dozen specimens from the Pelews, in comparison with the type specimen, we find the smaller size to be constant, and cannot unite them with those noticed by Mr. Coues, after the two specimens from Cape St. Lucas, Lower California (coll. Xantus) in the collection of the Smithsonian Institution, and the figure given by Mr. Elliot (Introd. B. N. Am. fig. head, natural size). In coloration there seems to exist no considerable 
difference from $P$. opisthomelas: the dark colour extends in both below the lores and eyes; the under tail-coverts are fuliginous black, \&c. In respect to colour our species is also closely allied with $\boldsymbol{P}$. anglorum, Temm., but this latter has only the outer row of the under tail-feathers black on the outer web, and is nearly as large as $P$. opisthomelas.

To this last-named species belongs Puffinus obscurus, Vieill. Gal. Ois. t. 301 (tab. sol. excl. descr.).

We are not fully acquainted with the geographical distribution of $P$. dichrous, having only seen specimens from the Pelews and M'Kean's Island. Schlegel notices it from Bourbon, and also a specimen (said to be one of the types of 'Temminck's $P$. obscurus) from the Atlantic; but this latter locality requires confirmation.

This species is the P. obscurus of $\mathbf{K} u h l$, Temminck, and Schlegel, and perhaps of Degland and Gerbe, who confounded it, as nearly all authors do, with the true Pr. obscura of Latham and Gmelin. In respect to this latter very confused species we are able to give some notes, which perhaps will be of some use :-

\section{Puffinus obscurus (Gmel.).}

Dusky Petrel, Lath. Gen. Syn. iv. p. 416 (Christmas Island).

Procellaria obscura, Gmel. S. N. p. 559 (ex Lath.) ; Bechst. Lath. Trans. iii. p. 364 (inaccur.); Lath. Ind. Orn. ii. p. 828, Vieill. Enc. Méth.i. p. 81 (ex Lath.); id. Gal. Ois. p. 230 (nec tab. 30 i).

Puffinus obscurus, Reichb. Schwimmrög. Novit. t. vi. f. 22.50, 2251 (ex Mus. Dresd.); Bp. Consp. ii. p. 202 (ex Lath., syn. excl.); Verr. MS. (Madagascar); Hartl. Madag. p. 84 (excl. syn.).

Nectris gama (nec Bp.), Hartl. Madag. p. 84 (excl. syn.).

? Procellaria obscura, Schleg. \& Pollen, Rech. Faune Madag. p. 161 (syn. Nectr. gama, Hartl.).

This species, black above, white beneath, may be distinguished at once by the uniform pure white under tail-coverts; the black on the upper parts does not reach beyond the loreal and a ricular regions ; the tarsus and feet, are yellowish, with the exterual toe and outside of tarsus partially black.

M. Jules Verreaux, in his excellent manuscript notes, describes this species from Madagascar, Bourbon, and Mauritius, whence he received specimens through Telfair, Desjardin, and Syanzin. A specimen in the Stuttgart Museum, from Madagascar, has been referred by $\mathrm{Dr}$. Hartlaub to Nectris gama; but the true $N$. gama, $\mathrm{B}$. (Consp. ii. p. 202) is different, although a very doubtful species.

I have examined the Madagascar specimen in the Stuttgart Museum (labelled $P$. baillonii), and noticed the external lateral under tail-coverts on the outer web black.-F.

P. obscurus inhabits the Indian Ocean (Christmas Island, Latham; Madagascar, B urbon, Mauritius, Verr.). As regards its occurrence in Europe, as first mentioned by Temminck ('Alpes du Pıémont ${ }^{\circ}$ ), and America, we have no trustworthy evidence. Schlegel (Vogels van Nederland, p. i85) savs, "several times obtained on our shores;" but later (Mus. P.-B. B. p. 30), "Je ne connais pas 
d'exemple bien constaté que cet oiseau ait été observé sur les côtes d'Europe."

Indeterminable are the following references, usually referred to $P$. obscurus :-

Puffinus obscurus, Schleg. Vog. van Nederl. p. 585 (perhaps $P$. assimilis, G.) ; id. Rev. Crit. p. cxxxiii ("Nördliches Europa!”).

Nectris obscura, Keys. \& Blas. Wirbelth. Eur. p. 94 ("Mittelländisches Meer, Südküste Nordamerika's").

Puffinus obscurus, Linderm. (Vögel Griechenl. p. 171) and Krüper (Journ. f. Orn. 1863), belongs to P. anglorum, Ray, or the hardly different Mediterranean form P. yelkuan, Acerbi.

Puffinus obscurus, Gould (B. Eur. pl. 444), we have had no opportunity of comparing.

Puffinus assimilis, Gould, P. Z. S. 1837 , p. 186 ; id. B. of Austr. vii. t. 59; Reichb. Schwimmv. f. 2600 (ex Gould); Gray, Gen. of B. iii. p. 647 (syn. P. nugax, Sol. MS.).

Puffinus nugax, Bp. Consp. ii. p. 205; Schleg. Procell. p. 31; Gould, Handb. B. Austr. ii. p. 458; Finsch \& Hartl. Centr. Polyn. p. 242 ; Coues, Proc. Acad. Phil. 1864, p. 141.

Puffinus assimilis, Gray, Ibis, 1862, p. 244.

Puffinus obscurus, Gray, B. Trop. Isl. p. 55 ; Finsch, Journ. f. Orn. 1870 , p. 371.

Like $P$. obscurus, but smaller ; the under tail-coverts also uniform white; but the white of the underparts mounts up on the sides of the head, including loreal and auricular regions; tarsi greenish yellow, webs bright chrome-yellow.

In the Pacific seas of Australia and New Zealand.

[ I was wrong in my note (cited above) in stating this to be the true P. obscurus, Gmel.-F.]

[In the Royal Museum at Munich I have examined two specimens of a Puffinus from Madeira, brought home by H.G. the Duke of Leuchtenberg, which apparently belong to this species. They ayree very well with the characters noticed above. The under tail-coverts are white, only the lateral ones at the base of the outer web dark, but this hidden.-F.]

Puffinus auduboni, Finsch.

Puffinus obscurus, Audub. Syn. B. N. Am. 1839, p. 339 ; Baird, B. N. Am. p. 835 ; Coues, Proc. Acad. Phil. 1864, p. 137 ; Licht. Nomencl: p. 100.

Like $P$. assimilis, but the under tail-coverts fuliginous-black; the anterior lateral under tail-feathers are on the outer vane black, on the inner white; the white on the sides of the head extends, as in $P$. as similis, not below the level of the eyes; bill deep leaden blue; feet and legs coloured as in P. anglorum.

This species inhabits the Atlantic Ocean, occurring not unfrequently along the shores of the Southern United States and in the Gulf of Mexico; it is abundant in the Bahamas and Bermuda. 
I have examined a fine specimen in the Berlin Museum, collected by Mr. Deppe at Cape Florida (mentioned by Bonaparte as $\boldsymbol{P}$. foridanus, Consp. ii. p. 204). "P. l'herminieri, Less.," cited by Bonaparte (Consp. pp. 189 and 204) as synonymous with this species, is not to be found in Lesson's 'Tr. d'Orn.'

We have before us a specimen of Puffinus from the Viti Islands, collected by Dr. Gräffe. It is the one mentioned by us as $P$. nugax (Centr. Polynesien, pp. 243 and 250, t. iii. f. 5), finserted only on the authority of Dr. Gräffe, who sent eggs from Viti Levu marked as those of this species. On comparing the specimen, the only one we have yet seen from this locality, we find it not to be the true P. assimilis, but a different species, which we are not able to make out. It resembles most $P$. auduboni, in having also the white not extended beneath the loreal region and eye, and the under tail-coverts fuliginousblack; but the specimen is smaller. Further researches must be made to settle this difficult species with certainty; we do not feel able to do so, having but a single specimen before us.

\begin{tabular}{|c|c|c|c|c|c|c|c|c|c|c|c|}
\hline $\begin{array}{l}\text { Long. } \\
\text { tot. }\end{array}$ & $\begin{array}{l}\text { Long. } \\
\text { al. }\end{array}$ & & Ap. al. & Caud. & $\begin{array}{l}\text { Rostr. } \\
\text { a fr. }\end{array}$ & $\begin{array}{l}\text { Rostr. } \\
\text { a rict. }\end{array}$ & $\begin{array}{l}\text { Alt. } \\
\text { rostr. } \\
\text { a bas. }\end{array}$ & $\begin{array}{l}\text { Tub. } \\
\text { nas. }\end{array}$ & Tars. & $\begin{array}{l}\text { Dig. } \\
\text { med. } \\
\text { (sine } \\
\text { ung.). }\end{array}$ & \\
\hline c. $11 \frac{1}{2}-13$ & $7 \stackrel{\prime \prime \prime}{2-7}$ & & 3 & $10-3$ & $111 \frac{1}{2}-12 \frac{1}{2}$ & $15-16 \frac{1}{21}$ & $3 \frac{34}{4}-4$ & "IIIII & $16-17$ & $16-17$ & dichrous (Pelew, 11 spec.). \\
\hline ... & & $11]^{-}$ & $\begin{array}{ll}2 & 4\end{array}$ & $\begin{array}{lll}2 & 11\end{array}$ & {$\left[\begin{array}{ll}-2 & 11\end{array}\right.$} & $16^{2}$ & 4 & 2 & 16 & 15 & - (M'Kean Is \\
\hline 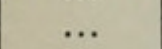 & & 3 & & 25 & $13 \frac{1}{2}$ & 18 & 4 & & 20 & 20 & - (obscurus, ap. Kंuhl). \\
\hline 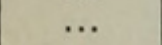 & 7 & & 23 & 211 & & $\cdots$ & $3 \frac{1}{2}$ & 2 & 17 & 16 & - (-, Schleg., Bourbon). \\
\hline$\cdots$ & 7 & & 23 & 210 & $12 \frac{1}{2}$ & & 3 & 2 & 17 & 16 & $-(-$, Atl. Oc. $)$. \\
\hline$\ldots$ & 9 & & $\cdots$ & $3 \cdot 75$ & $1 \cdot 40^{\prime \prime}$ & 24 & .. & $\ldots$ & $1 \cdot 80^{\prime \prime}$ & $2 \cdot 10^{\prime \prime}$ & opisthomelas (ex Coues). \\
\hline$\cdots$ & 8 & 7 & $\cdots$ & 34 & 16 & 20 & $\cdots$ & $\ldots$ & 19 & 19 & - (obscurus, Vieill. ap. pl.). \\
\hline$\cdots$ & $\cdots$ & & $\cdots$ & $\cdots$ & 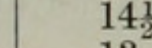 & $\ldots$ & 4 & $\cdots$ &  & & -- (ex Elliot's fig.). \\
\hline$\cdots$ & 7 & 9 & $\ldots$ & . & 13 & & $\cdots$ & .. & 17 & 16 & $\begin{array}{l}\text { auduboni (Florida, Berl. } \\
\text { Mus.). }\end{array}$ \\
\hline$\cdots$ & 8 & 0 & & $4 \cdot 25$ & $1 \cdot 25^{\prime \prime}$ & $1 \cdot 70^{\prime \prime}$ & $\cdots$ & $\cdots$ & $1 \cdot 60^{\prime \prime}$ & & - (obscurus, Coues). \\
\hline & $66-6$ & 112 & $5-3 \quad 92$ & $2-27$ & $711-12$ & $\ldots$ & ... & $\ldots$ & $16-17$ & $15-17$ & assimilis, Gould (ex Schleg.). \\
\hline$\cdots$ & $63-6$ & 6 & $\ldots$ & $\ldots$ & 11 & $\ldots$ & $\ldots$ & $\ldots$ & 15 & $\ldots$ & Madeira. \\
\hline$\cdots$ & 7 & 9 & $\cdots$ & 32 & 14 & 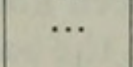 & & & 17 & $\ldots$ & obscurus, Gmel. (ex Gama, \\
\hline$\cdots$ & 7 & 0 & 25 & 26 & 14 & $19 \frac{1}{2}$ & $3 \frac{1}{4}$ & $1 \frac{1}{2}$ & 16 & 16 & spec.?, Vitis. \\
\hline
\end{tabular}

46. Sterna longipennis, Nordm.; F. \& H. Orn. Centr. Polyn. p. 220 (syn. part.).

One specimen from the Pelews (Capt. Heinsohn), not in full plumage. Only the occiput and nape are black; forehead brownish, with darker shaft-stripes; the feathers of the grey upper parts with narrow pale edges; the three outer tail-feathers on the outer web grey, the remainder only washed with grey ; the small wing-coverts along the humerus dark brown, forming a longitudinal band; bill black ; feet reddish.

Long al. rectr. ext. rectr.interm. culm. altit. ad bas. tars. dig. med.

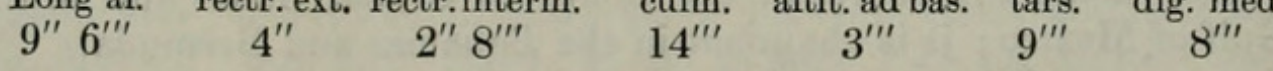


The specific differences of St. frontalis, Gray, from New Zealand, which we formerly erroneously confounded with this species, have been pointed out already (Journ. f. Orn. 1870, p. 365).

The Pelew Islands are a new locality for St. longipennis.

47. Sterna melanauchen, Temm.; F. \& H. Centr. Polyn. p. 224.

Two adult specimens, one from the Pelews (Capt. Heinsohn), the other from Uap (Kubary). Both localities are new for this species.

48. Sterna lunata, Peale; Hartl. P.Z. S. 1867, p. 331 ; H. \& F. $i b .1868$, pp. $9 \& 118$.

One specimen from Pelew (Capt. Peters).

49. Anous stolidus (L.); H. \& F., P. Z. S. 1868, p. 9 (Pelew).

Seven specimens from the Pelews (Capts. Heinsohn and Peters). They agree exactly with specimens from N. America and Africa, but vary in the intensity of the dark colouring, and especially as regards the coloration of the head. In some the front and forehead are greyish white, changing into pale grey on the occiput and nape, and into pale ashy grey on the hind neck; in others the whole surface of the head is pale brownish grey. In one paler-coloured, apparently younger, specimen the head is uniform sooty brown like the neck, but mixed with single white feathers, which, above the black loreal regions, form an indistinct white supercilium. In all the specimens the lower half of the eye is margined narrowly with white; the legs and toes are blackish, the membranes lighter.

As regards the variation in size in this species, we must remark that the specimen from North America spoken of in Ornith. Centr. Polyn. p. 236, has not full-grown remiges, the first being just developing; from this cause the wing is much shorter.

We append the measurements of the Pelew specimens, to show the individual variation amongst specimens from the same locality.

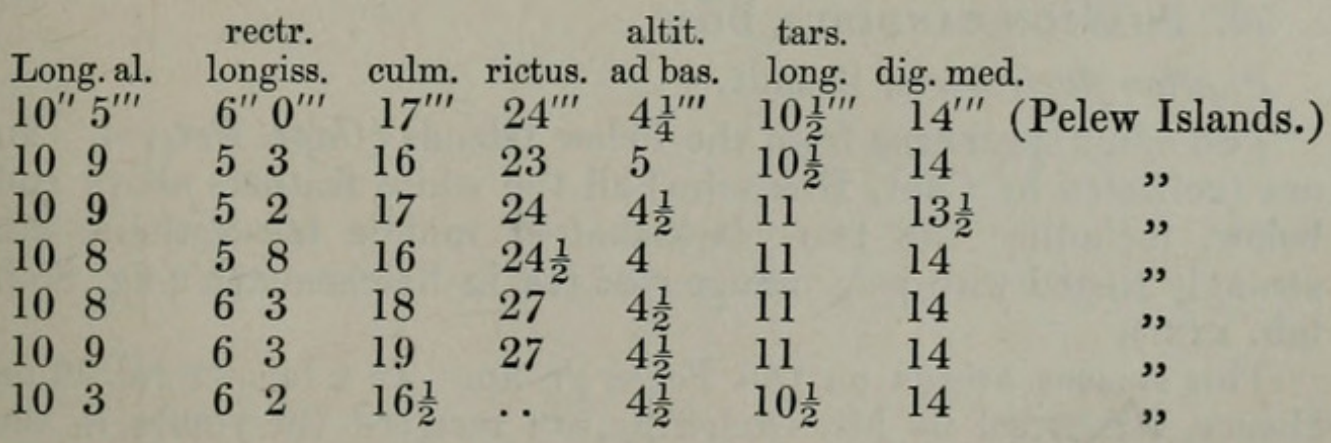

50. Anous tenuirostris (Temm.).

? Sterna senex, Cranch in Tuckey's Voy. Zaire (1818), App. p. 407.

Sterna tenuirostris, Temm. (nec Rüpp., nec Heugl.) Pl. Col. 202 ; (Temm.) Kittl. Kupfert. Heft iii. (1833), p. 28, f. 2, tab. 36. f. 1 (opt.).

Proc. Zool. Soc.-1872, No. VIII. 
114 BIRDS FROM THE PELEW AND MACKENZIE ISLANDS. [Jan. 16,

Anous leucocapillus, Gould, P. Z. S. 1845, p. 103; Finsch \& Hartl. Orn. Centr. Polyn. p. 237.

Sterna tenuirostris et S. leucocapilla, Schleg. Mus. P.-B. Sterna, p. 37 .

Eight specimens from the Pelew Islands (Capts. Heinsohn and Peters), where this species has not been hitherto noticed. This species differs from $A$. stolidus in its smaller size, the slender and nearly straight bill, the darker, more sooty-black coloration, and the purer, silvery-white surface of the head. In most of the specimens there is a dark greyish tinge on the temporal region, the hind neck and sides of neck. The younger bird is more of a sooty-brown coloration (as in $A$. stolidus), and has the upper wing-coverts partially margined with bright umber-brown. A still younger bird has the front and vertex already as pure white as the old one; and this is also seen in the nestling young, which is covered with black down. The legs are pale, as in A. stolidus, brownish or yellowish brown, in some yellowish. We see no reason for separating $A$. leucocapillus, Gould, from A. tenuirostris, Temm. The dimensions noticed by Prof. Schlegel are not sufficient to distinguish two species.

Long. al. rectr. long. culm. rict. altit. rostr. tars. dig. med. $8^{\prime \prime}-8^{\prime \prime} 9^{\prime \prime \prime} \quad 4^{\prime \prime}-4^{\prime \prime} 7^{\prime \prime \prime} \quad 18-21^{\prime \prime \prime} \quad 24-27^{\prime \prime \prime} \quad 3 \frac{1}{4}-4^{\prime \prime \prime} \quad 9-9 \frac{1}{2}{ }^{\prime \prime \prime} \quad 11-12 \frac{1}{2}$ "' (Pelew, 8 spec.)

Kittlitz gives a very accurate representation of this species (Kupfert. t. 36. f. 1), which he collected in the Carolines and also near St. Helena.

51. Gygis alba (Sparrm.) ; Hartl. P.Z. S. 1867, p. 832 ; H. \& F. ibid. 1868 , pp. $9 \& 118$.

From the Pelews (Capt. Peters), and a beautiful pair (male and female) from Uap, where Mr. Kubary notices the species as rare.

In the Eastern Carolines, observed by v. Kittlitz on the island of Ualan (Denkwürdigk. i. p. 382).

52. Рhä̈ton candidus, Briss.

Phaëton flavirostris, Brandt.

Two adult specimens from the Pelew Islands (Capt. Peters). In one (collected by Capt. Heinsohn) all the white feathers above and below, including the two black-shafted middle tail-feathers, are strongly tinged with pale orange-rose (as in Reichenbach's fig. 852, tab. xxx.).

This species breeds on the Pelew group. In a former collection thence, forwarded by Mr. Godeffroy, we received the young in the first plumage.

The 'Novara' expedition procured a specimen (in October) on the Stewart group; and v. Kittlitz observed it in Ualan, Eastern Carolines (Denkwürdigk. einer Reise \&c. i. p. 382).

53. Carbo melanoleucus, Gray; H. \& F., P.Z.S. 1868, p. 9.

Two adult specimens from the Pelew Islands (Capt. Peters). 


\section{$2 \mathrm{BHL}$ Biodiversity Heritage Library}

Newton, Alfred. 1872. "January 16, 1872." Proceedings of the Zoological Society of London 1872, 23-114. https://doi.org/10.1111/j.1469-7998.1872.tb00487.x.

View This Item Online: $\underline{\text { https://www.biodiversitylibrary.org/item/90880 }}$

DOI: https://doi.org/10.1111/j.1469-7998.1872.tb00487.x

Permalink: https://www.biodiversitylibrary.org/partpdf/73722

\section{Holding Institution}

Natural History Museum Library, London

\section{Sponsored by}

Natural History Museum Library, London

\section{Copyright \& Reuse}

Copyright Status: Public domain. The BHL considers that this work is no longer under copyright protection.

This document was created from content at the Biodiversity Heritage Library, the world's largest open access digital library for biodiversity literature and archives. Visit BHL at https://www.biodiversitylibrary.org. 